This document classified by:

R. M. Smi thers, Manager

Waste Processing Section

\title{
RADIOACTIVE LIQUID WASTES DISCHARGED TO GROUND IN THE 200 AREAS DURING THE FIRST THREE QUARTERS OF 1973
}

\author{
J. D. Anderson \\ Operations Analysis \\ Waste Processing Section \\ Manufacturing and Waste Management Division
}

January 15,1974

Operated for the Atomic Energy Commission by

Atlantic Richfield Hanford Company under Contract AT(45-1)-2130 


\section{DISCLAIMER}

This report was prepared as an account of work sponsored by an agency of the United States Government. Neither the United States Government nor any agency Thereof, nor any of their employees, makes any warranty, express or implied, or assumes any legal liability or responsibility for the accuracy, completeness, or usefulness of any information, apparatus, product, or process disclosed, or represents that its use would not infringe privately owned rights. Reference herein to any specific commercial product, process, or service by trade name, trademark, manufacturer, or otherwise does not necessarily constitute or imply its endorsement, recommendation, or favoring by the United States Government or any agency thereof. The views and opinions of authors expressed herein do not necessarily state or reflect those of the United States Government or any agency thereof. 


\section{DISCLAIMER}

Portions of this document may be illegible in electronic image products. Images are produced from the best available original document. 
COATENTS

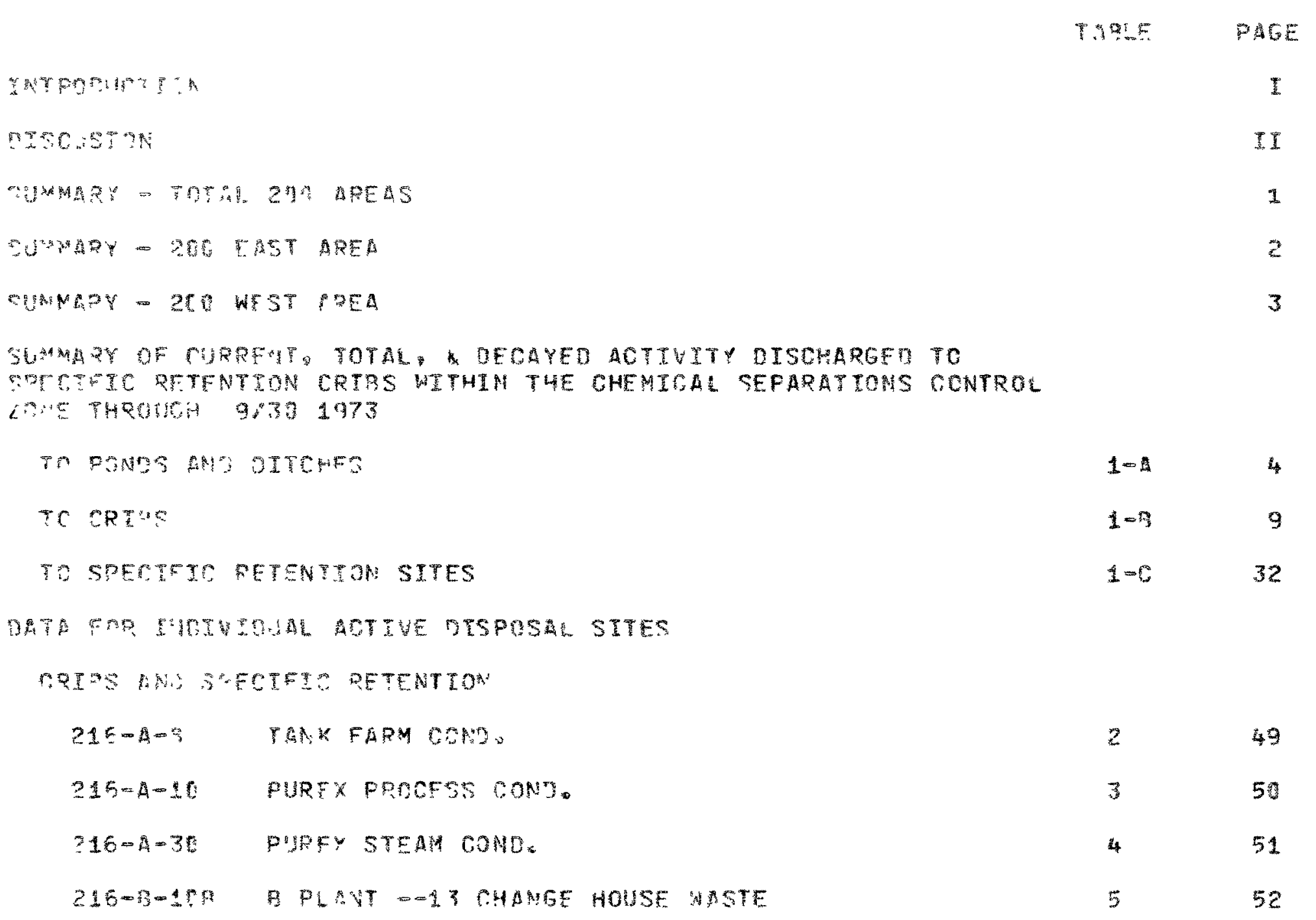


CONTENTS

\begin{tabular}{|c|c|}
\hline $216-9-x 2$ & P FLANT PROCESS COND. \\
\hline $216-8-5$ & TPS- 1 CONDENSATE \\
\hline $216-8-55$ & P PLANT STPAM COND. \\
\hline $216-8-57$ & ITS-2 CONDENSATE \\
\hline $21 \div-C=7$ & 209-E CRITICAL MASS LAB \\
\hline $216-5-2 n$ & 222-S LABORATORY WASTE \\
\hline $216-T-19$ & 242-T EVAPORATOR COND. \\
\hline $215-7-12$ & $234-5$ PROCESS AND LAB MASTE \\
\hline $216-7-16$ & 231 WASTE \\
\hline $16-Z=18$ & PLUTONTUM RECLAHATION WASTE \\
\hline
\end{tabular}

TABLE PAGE

PONDS AND DITCHES

$\begin{array}{ll}216-A-25 & \text { GAQLE MCUNTAIN POHD AND OITCHFS (SUMMARY) } \\ 216-A-25 & \text { PUREX COOLING WATER } \\ 216-A-25 & \text { AR VAULT COOLING WATER } \\ 216-3-3 & \text { B PCND AND DITCHFS ISUMMARY } \\ 216-B-3 & \text { 3 PLANT COOLING WATER } \\ 216-8-3 & \text { ITS-1 COOLING WATER }\end{array}$

$21 \quad 68$




\begin{tabular}{|c|c|}
\hline $2 \cdot \frac{1}{1} b=3$ & PTS-2 OCORING WATER \\
\hline $215-3+3$ & PUPEX CHEMECAL SEWER \\
\hline $215-9=53$ & 3 PLANT TRENCH (SUMMARY) \\
\hline $216-3-63$ & 9 FLANT CHEM SEWEQ \\
\hline $216-5-11$ & RESCK CHEM SEHER DTTCH \\
\hline $215-5-19$ & $222-5$ LAB POND \\
\hline $216-8=4$ & POND \\
\hline $216-y-1 t$ & " PCNO ANO DITCHES PSUMMARY \\
\hline $216-v-16$ & BUNORY \\
\hline $218-1-10$ & $241-5 X$ COOLING WATER \\
\hline $21 E-U-10$ & $231-2$ WASTE WATER \\
\hline $216-U-18$ & $234-5$ WASTE WATER \\
\hline
\end{tabular}

$\begin{array}{cc}\text { TAGLE } & \text { PAGE } \\ 22 & 69 \\ 23 & 70 \\ 24 & 71 \\ 25 & 72 \\ 25 & 73 \\ 27 & 74 \\ 28 & 75 \\ 29 & 76 \\ 30 & 77 \\ 31 & 78 \\ 32 & 79 \\ 33 & 80 \\ & 91\end{array}$




\section{INTRODUCTION}

This document is issued quarterly for the purpose of summarizing the radioactive liquid wastes that have been discharged to ground in the 200 Areas. In addition to data for the first three quarters of 1973, cumulative data since plant startup are presented. Also in this document is a listing of decayed activity to the various plant sites. 


\section{DISCUSSION}

The format of this report has been changed considerably from the preceding reports so that all of the information on each site can be found on one page. All of the information found in Tables 1 , 2, 3, and the Appendix has been combined and is now located in Table 1. The data in Table 1 contains an estimate of the radioactivity discharged to individual ponds, cribs, and specific retention sites within the Manufacturing and Waste Management Division during the first three quarters of 1973 and from startup through September 30, 1973; an estimate of the decayed activities from startup through September 30, 1973; the location and reference drawings of each disposal site; and the usage dates of each disposal site. The estimates for the radioactivity discharged and for decayed activities discharged from startup through September 30, 1973 are based upon Item 4 of the Bibliography. The volume of liquid discharged to the ponds also includes major nonradioactive streams.

An overal1 summary is presented on page 1 giving the radioactive 1 iquid wastes discharged to ground during the first three quarters of 1973 and since startup (for both total and decayed depositions) within the Manufacturing and Waste Management Division control zone (200 Area plateau). Overall summaries are also presented on page 2 for 200 East Area and on page 3 for 200 West Area.

The wastes discharged during 1973 to each active disposal site are detailed on a month-to-month basis in Tables 2 through 33 along with the monthly maximum concentration and average concentration data. An estimate of the radioactivity discharged to each active site along with the remaining decayed activities are also found in these tables. This places all of the data on each active disposal site in one table.

The data contained in this report supersedes that contained in the first half report (ARH-2806 2Q, unclassified, October 19, 1973, J. D. Anderson, "Radioactive Liquid Wastes Discharged to Ground in the 200 Areas During First Half 1973"). It should also be noted that the plutonium value for the $216-Z-9$ site has been reduced from 150 kilograms to 38.5 kilograms based upon new analytical data.

The data presented in the tables are to be read as shown by the following examples:

$$
\begin{aligned}
.241 \mathrm{E}+07 & =0.241 \times 10^{7} \\
.241 \mathrm{E}-07 & =0.241 \times 10^{-7}
\end{aligned}
$$


THE RADIOACTIVF LIQUTD WASTES OISCHARGED TO GROUND WERE

\begin{tabular}{|c|c|c|c|c|c|}
\hline FROM $1 / 1$ TO & $9 / 30 \quad 1973$ & $\begin{array}{l}\text { TO PONDS } \\
\text { AND DITCHFS }\end{array}$ & $\begin{array}{l}\text { TO } \\
\text { CRIBS }\end{array}$ & $\begin{array}{l}\text { TO SPECIFIC } \\
\text { RETENTION SITES }\end{array}$ & TOTAL \\
\hline $\begin{array}{l}\text { VOLUME } \\
\text { PU } \\
\text { BETA } \\
\text { SR-90 } \\
R U-106 \\
C S-137 \\
C D-60 \\
U \\
U-233\end{array}$ & $\begin{array}{l}(L) \\
\text { (GM) } \\
\text { (CI) } \\
\text { (CI) } \\
\text { (CI) } \\
\text { (CI) } \\
\text { (CI) } \\
(\mathrm{KGM}) \\
(\mathrm{GM})\end{array}$ & $\begin{array}{r}.752 E+10 \\
<.903 E+01 \\
<.348 E+01 \\
<.567 E-01 \\
<.147 E+00 \\
<.160 E+00 \\
<.561 E-01 \\
<.552 E+02\end{array}$ & $\begin{array}{r}.225 E+09 \\
.335 E+03 \\
.774 E+02 \\
.107 E+02 \\
.502 E+01 \\
.190 E+102 \\
<.366 E+00 \\
<.302 E+01\end{array}$ & $\begin{array}{l}.366 E+06 \\
.127 E+04\end{array}$ & $\begin{array}{r}.774 E+10 \\
.161 E+04 \\
.819 E+02 \\
.107 E+02 \\
<.516 E+01 \\
.191 E+02 \\
<.422 E+00 \\
<.582 E+02\end{array}$ \\
\hline
\end{tabular}

\section{THROUGH 9/3D 1973 (FROM START-UP)}

$\begin{array}{llrlrl}\text { VOLUME } & \text { (L) } & .478 E+12 & .275 E+11 & .185 E+09 & .506 E+12 \\ \text { PU } & \text { (GM) } & .917 E+04 & .930 E+05 & .898 E+05 & .192 E+06 \\ \text { BETA } & \text { (CI) } & .259 E+05 & .298 E+07 & .105 E+07 & .429 E+07 \\ \text { SR-90 } & \text { (CI) } & .176 E+04 & .319 E+05 & .118 E+05 & .454 E+05 \\ \text { RU-106 } & \text { (CI) } & <.377 E+04 & .253 E+06 & .301 E+06 & .558 E+06 \\ C S-137 & \text { (CI) } & .630 E+03 & .177 E+05 & .322 E+05 & .506 E+05 \\ \text { CO-60 } & \text { (CI) } & <.784 E+02 & <.528 E+03 & .144 E+03 & <.751 E+03 \\ U & \text { (KGM) } & <.658 E+04 & .638 E+05 & .480 E+05 & .118 E+06 \\ U-233 & \text { (GM) } & <.489 E+03 & .479 E+03 & <.967 E+03\end{array}$

DECAYED THROUGH $9 / 301973^{\circ}$ (FRCM START UP)

\begin{tabular}{|c|c|c|c|c|c|}
\hline $\begin{array}{l}\text { VQLUME } \\
\text { PU } \\
\text { BETA } \\
S R-90 \\
R U-106 \\
C S-137 \\
C O-60 \\
U \\
U-233\end{array}$ & $\begin{array}{l}(L) \\
(G M) \\
(C I) \\
(C I) \\
(C I) \\
(C I) \\
(C I) \\
(K G M) \\
(G M)\end{array}$ & $\begin{array}{r}.478 E+12 \\
.917 E+04 \\
.559 E+04 \\
.152 E+04 \\
<.208 E+02 \\
.509 E+03 \\
<.378 E+02 \\
<.558 E+04 \\
<.489 E+03\end{array}$ & $\begin{array}{r}.275 E+11 \\
.93 E E+05 \\
.811 E+05 \\
.207 E+05 \\
.143 E+04 \\
.123 E+05 \\
. .143 E+03 \\
.638 E+05 \\
.479 E+03\end{array}$ & $\begin{array}{l}.185 E+09 \\
.898 E+05 \\
.593 E+05 \\
.796 E+04 \\
.179 E+03 \\
.213 E+05 \\
.182 E+02 \\
.480 E+05\end{array}$ & $\begin{array}{r}.506 E+12 \\
.192 E+06 \\
.146 E+06 \\
.302 E+05 \\
.163 E+04 \\
.346 E+05 \\
.199 E+03 \\
.11 B E+06 \\
.967 E+03\end{array}$ \\
\hline
\end{tabular}


THF RAOINACTIVE LIQUID WASTFE EISCHRRGED TO GROUND WERE

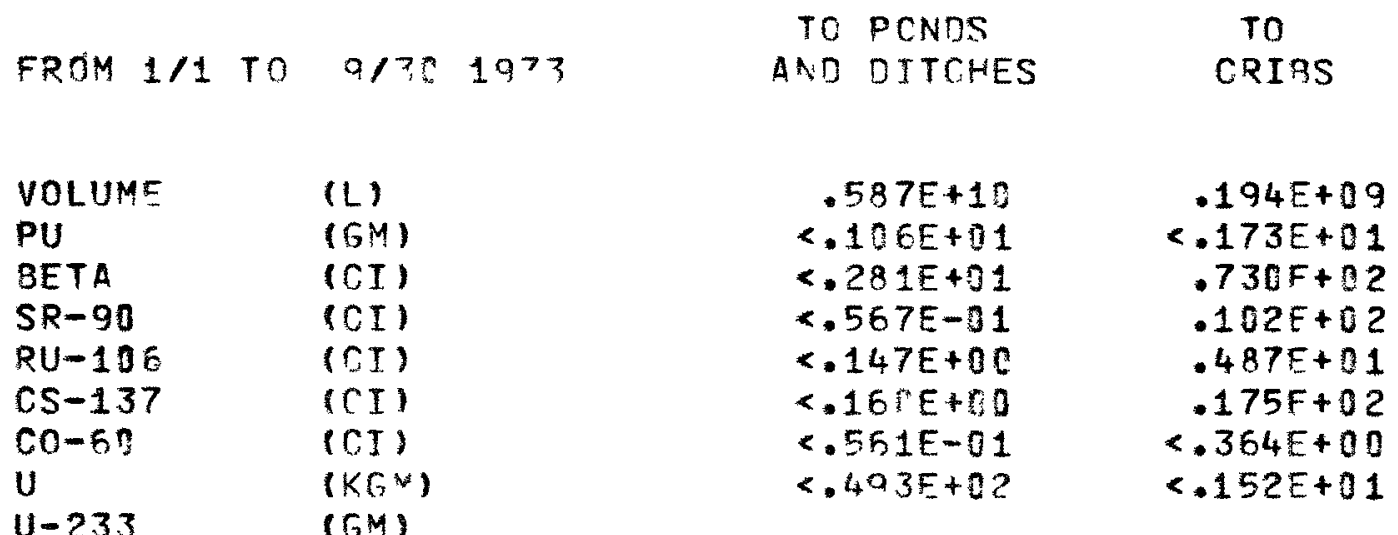

TO SPECIFIC

RETENTION SITES

TOTAL

$.607 F+10$

$<.279 E+01$

$.758 E+02$

$103 E+02$

$<.532 E+01$

$177 F+02$

$<.420 E+00$

$<.538+12$

THROUGH $9 / 301973$ (FROM START-UP)

\begin{tabular}{|c|c|c|c|c|c|}
\hline VOLUME & (L) & $.263 F+12$ & $.161 E+11$ & $.103 E+09$ & $.279 E+12$ \\
\hline PU & $(G M)$ & $<.662 F+03$ & $.105 E+05$ & $.437 E+03$ & $.116 E+05$ \\
\hline BETA & (CI) & $.252 E+06$ & $.176 E+07$ & $.100 E+07$ & $.302 E+07$ \\
\hline$S R-9 D$ & (CI) & $.159 E+104$ & $.226 E+05$ & $.116 E+05$ & $.357 E+05$ \\
\hline$R U-106$ & (CI) & $<.306 E+04$ & $.237 E+06$ & $.301 E+06$ & $.541 E+6$ \\
\hline $\operatorname{cs}-137$ & $(C T)$ & $.510 E+03$ & $.105 E+05$ & $.157 E+05$ & $.268 E+05$ \\
\hline $\mathrm{co}-60$ & $(C I)$ & $<.641 E+02$ & $<.430 E+03$ & $.115 E+03$ & $<.609 E+13$ \\
\hline $\mathrm{v}$ & $(K G M)$ & $<.101 E+04$ & $.277 E+15$ & $.467 E+05$ & $55 E+15$ \\
\hline$u-233$ & $(r M)$ & $<.489 E+03$ & $.4795+23$ & & $<.967 E+133$ \\
\hline
\end{tabular}

DECAYFN THFRUGH $9 / 301973$ (FRCF START UP)

\begin{tabular}{|c|c|c|c|c|c|}
\hline VOLUAE & (L) & $.263 E+12$ & $.161 F+11$ & - $103 E+09$ & $.279 E+12$ \\
\hline PU & $(G, M)$ & $<.562 E+03$ & $.105 E+05$ & $.437 E+03$ & $.116 E+05$ \\
\hline BFTA & (CI) & $.516 E+04$ & $.562 F+05$ & $.387 E+15$ & $.100 E+B 6$ \\
\hline$S R-90$ & $(C I)$ & $.140 E+04$ & $.144 F+05$ & $.781 E+04$ & $.236 F+05$ \\
\hline$R U-106$ & $(C I)$ & $<.199 E+02$ & - $138 E+04$ & $.178 E+03$ & $.158 E+04$ \\
\hline $\operatorname{cs}-137$ & $(r I)$ & $.421 E+03$ & $.766 E+04$ & $.107 E+05$ & $.188 E+05$ \\
\hline$c 0-50$ & (CI) & $<.313 E+02$ & $<.117 E+03$ & $.159 E+02$ & $<.1$ \\
\hline & $(K C M)$ & $<.101 E+04$ & $.277 E+05$ & $.467 E+05$ & 05 \\
\hline$v-233$ & $(G, M)$ & $<.489 E+03$ & $.479 E+13$ & & $7 E+03$ \\
\hline
\end{tabular}


THE RANIOACTTVE LIOUIO WASTFS MISCHARGED TO GROUND WERE

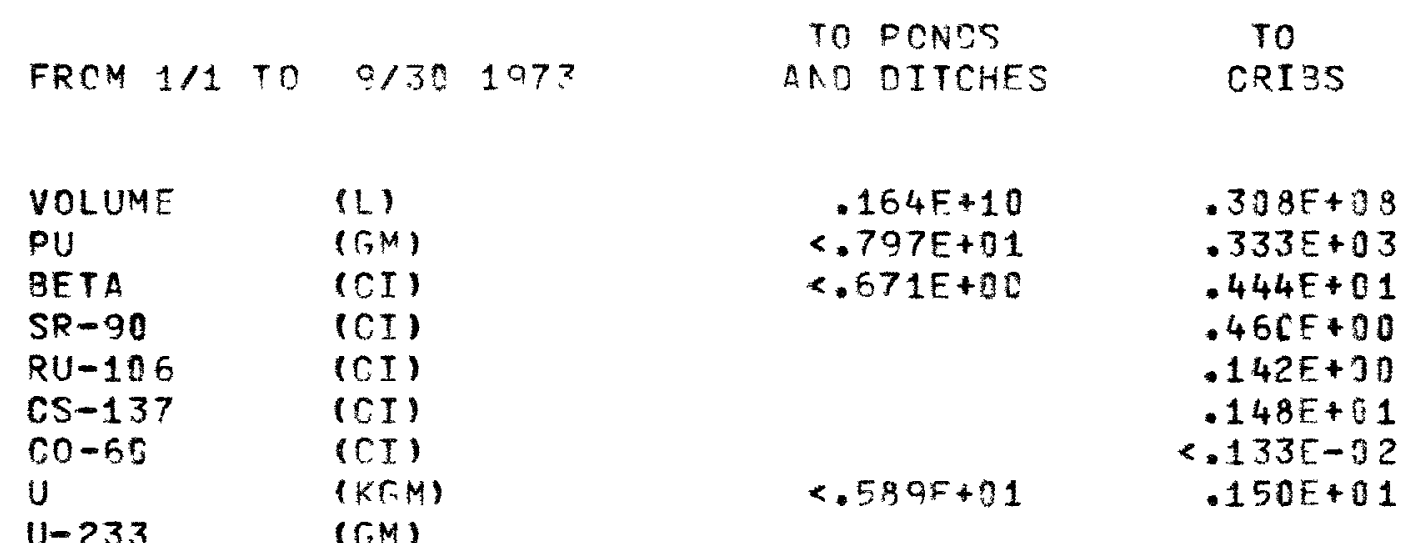

TO SFECIFIC PETENTION SITES

TOTAL

$\begin{array}{rr}.366 E+06 & .167 E+10 \\ .127 E+04 & .161 E+04 \\ & <.511 E+01 \\ & .460 E+00 \\ & .142 E+00 \\ & .148 E+01 \\ & <133 E-02 \\ & <.739 E+01\end{array}$

$.328 E+08$ - $894 \mathrm{E}+05$ $.470 E+05$ $.238 E+03$ $.902 E+03$ -155E+05 $.289 E+02$ - $129 E+C^{2}$

$00-60$

(CI)

u

$(C T)$

$<.558 E+04$

$.361 E+65$

$\left(\mathrm{GN}^{\mathrm{N}}\right)$
$.227 E+12$ $.130 E+06$ $.127 E+07$ $.973 E+04$ $.176 E+05$ $.238 E+05$ $<.141 E+13$ $.429 E+05$
$.114 F+11$

$.825 E+35$

$.249 F+35$

$.632 E+74$

$.530 F+02$

$.510 E+34$

$<.258 E+72$

$.361 E+05$
$.829 F+118$ . $894 E+05$ $.206 E+05$ $.147 E+03$ $.937 E+100$ $.106 E+05$ - $230 E+01$ $.129 E+14$
CS-13?

u

$u-233$
(CI)

(KCM)

$(r+1)$
$<.975 E+02$

$\times .558 E+04$
$.227 E+12$ - $130 E+156$

$.460 E+05$

$.658 E+04$

$.549 E+02$

$.158 E+05$

$<.346 E+32$

$.429 E+05$ 
SUYMARY OF CURFFAT, TOTAL, DECAYFD ACTIUITY DISCHARGEO TC FONDS AND DITCHES WITHIN THE FHFNITAL SFPARATION AREA CONTROL ZONE THROUGH $9 / 301973$

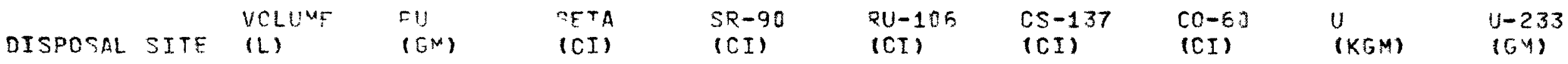

$21 E-A-25$

1973 SUM TOT SUM TOTRFSAY

$216-9-2-2=$

1972 Sin TOT EUM

TOTRERAY

$216-3-2-2 w$

$197^{2}$ SUM TOT SUM

TOTOECAY

$216-n-3$

GOQLE MOUNTAIN PCND. PLACED IN USF OFC 1957 AND IS STILL ACTIVE. APPRTYYMATF TCOREJNATES: N-5FF32, W-513ER TO N-54763, W-52052 TO N-50962, $h-47>49$ TC N-5183i, W-46647. QFFERENCE DFANINGS: H-2-66018, $1-2-3325$.

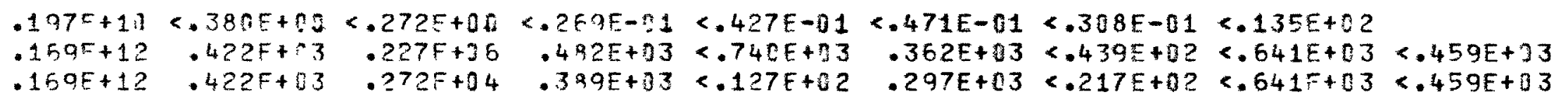

TERMTNISS CF 2-2-2 DTTCH. PLACED IN USE MAP 1970 AND PFMOVED FROM SERVICE AOV 1970. $\triangle E F R C X I M A T E$ COOROINATES: N-43692, h-46996 (HEAC) TC N-43250, W-4333A (TEPNINUS). DEFFREVRT ARANINCS: N-26CC-E SHT 26. THE VOLUME IS INCLUDFE TN THF $216-A-3$ POND.

$\begin{array}{lll}.14 E F+34 & .710 E+03 & .5 C 3 E+00 \\ .131 E+04 & .655 E+13 & .464 F+00\end{array}$

HEAD OF B-2-2 TITSH. PLACE? IN USF MAP 197 AND RE WGED FRCM SFPVICE

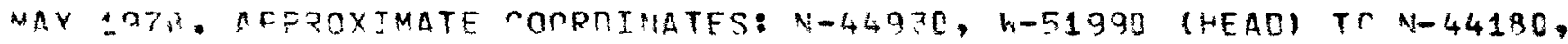
W-49F25 (TFFMINLS). PEFFRFNCF DRAHINGS: H-2-44500 SHT 5.

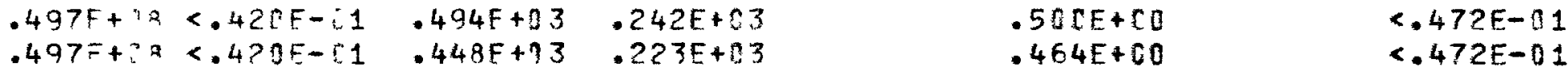

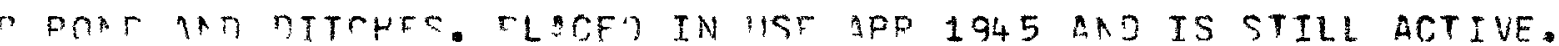
APPOSX MATE CONETINATES: N-43957, W-44787, N-42468, W-41745. FEFEPEACE DEAWINCS: H-2-2431, H-6-419.

$1977^{\circ} 5 U^{4}$ TOT SUM TOTEFOAY
$.3395+13<.5965+3$ $.914=+11<.39 E+3$ $.914 F+11<.239 F+13$
$.159 F+01<.152 E-01$ $.232 E+135$ - $643 E+3$
$152 \mathrm{E}+03$

$.128 E+63$
$<.103 E+00$
$.232 E+04$

$.716 E+11$
$<.688 E-01<.179 E-11<.332 E+32$

$.146 E+03<.198 E+02<.359 E+03<.300 E+02$ $.121 F+03<.945 F+01<.359 E+03<.300 E+02$ 
SUMMAQY OF CUPRENT, TOTAL, DECAYED ACTIVITY DISCHARGED TC FONOS AND DITCHES WITHTN THF CHEMICAL SEFARATION AREA CONTROL ZONE THRCUGH 9/30 1973

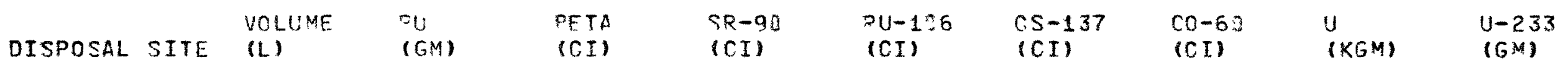

$216-9-63$

1973 SUM TOT SUM

TOTDECAY

$216-c-9$

1973 SUM TOT SUM

TOTEECAY

$21 E-N-4$

1973 SUM TOT SUY

TOTDFCAY

$216-N-6$

$1973 \sin$ TOT SUM

TOTEECAY

9. PLANT CHFM SEHFP, ETC. PLACEN IN USE MAR 1970 ANO IS STILL ACTIVE. APFPCXIMATE CCOPRINATES: N-45110, W-51793 \&HFADI TO N-44635, W-50254 (TERMINUS). REFERENCE DRAWINGS: H-2-4450I SHT 5.

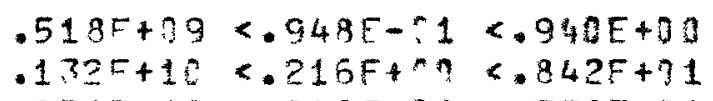

SEMTWORKS FOND. FLACFD IN USF JUN 1953 AND REMOVER FROM SERVICE SEP 1969. APPROXIMATE COCREINATES: N-42550, W-50740, N-42520, W-49840. RFFFRFNCE JRAWTNES: $\mathrm{H}-2-32523, \mathrm{H}-2-4110$.

$.1: 3 F+13<.338 F+03 \quad .794 F+32.514 E+31.523 E+n 1<.120 F+01<.385 E+03<.915 E+00$

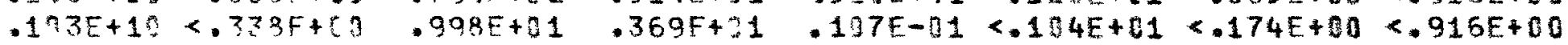

201 NCRTH E-FONC. DLACFO IN USF SFF 1944 AND REMOVEN FRON SEPVTCF JIIN 195?. AFOQOXIMATF COORDINATFS: N-54250, W-52900, N-5340D, W-62331. DFFFPARE TOAWTACS: H-2-32524.

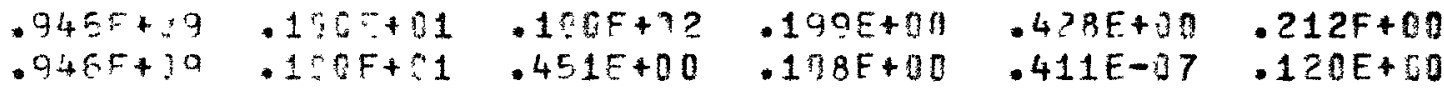

2OO NORTH R-PONC. PLACEO IN UEF SEP 1944 AND REMOVED FROM SERVICE JU:: 1952. AFROEYTMTF CONDIINATES: N-54275, w-60325, N-53600, $h-53800$. REFETENCF DFAUINES: H-2-32524. 
SUM*ARY IF CURFENT, TOTAL, DECAYCD ACTIVITY DISCHARGED TC PONDS AND DITCHES WTTHIN THF SHFMICAL SFRARATIJN ARFA CONTROL $70 N F$ THFCUGH 9/30 1973

$\begin{array}{lllllllll} & \text { VOLUMF } & \text { DU } & \text { OETA } & S R-9 i & R U-106 & C S-137 & C O-60 & U \\ \text { DISPOSAL SITE } & \text { (L) } & \text { (GM) } & \text { (CI) } & \text { (CI) } & \text { (CI) } & \text { (CI) } & \text { (CI) } & \text { (KGM) }\end{array}$

$216-5-10$

$1973 \sin$ TOT SUM TOTIFCAY

$216-5-1$

$197^{\circ} \mathrm{SIM}$ TOT TUM TOTDECAY

$216-5-16$

$1973 \mathrm{SUM}$ TOT CUM TOTMECAY

$216-S-17$

REDRX CHFM SEWER IITCH. PLAGFO IN USF FFE 1954 AND REMOVEN FRON SERVICE

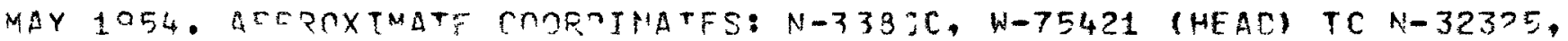
W-771:6. DEEFP-NCE TIRANINSS: H-2-325?5.

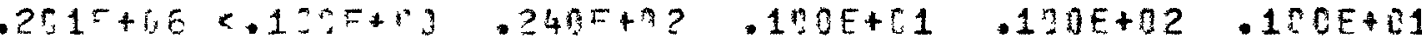
$.221 F+5<.1 .0+.3 .249 F+31.623 E+.00 .171 E-144.642 E+(0)$

PFDOY CHEM SENFP JTTCH. OLACFA IN USE MAY 1954 ANJ IS STILL ACTIVE. ADPREXIYATE COOPRINATFS: N-32693,W-7707: N-31750,W-76640.

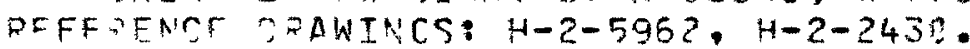

$149^{2}+17<.399 E-12<.595 F-0 ?$

$<.152 E+01$

$317 r+10<0279+50$

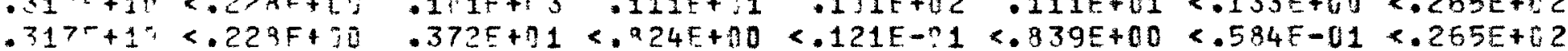

PFANX PCN NP.?. PLACEN IN USF SFP 1956 AND IS INATTIVE APPROXIMATF COORTINATE: N-21609, N-9114\% TO N-3292J, W-82193 TON-32920, W-9E910 TO

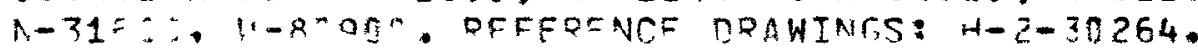

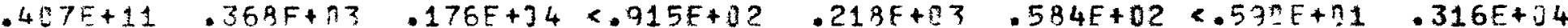

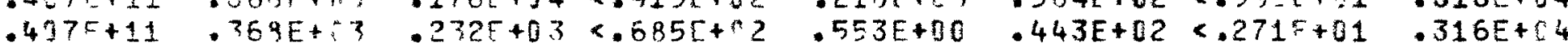

PENCX FNNP NC.1. JLACEO IN USF NOV 1951 ANT REMOVEM FROM SERVICF APP 1954.

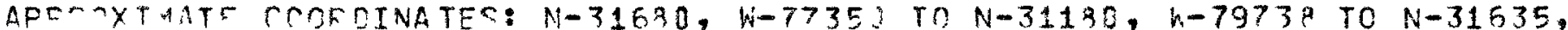
W-70789 TO N-329=9, $w-78265$ T0 N-32959, h-77350. PEFERENCE DQAHINGS: $H=2-2594$ 
SUMMARY IF CUFPENT, TOTAL, \& DECAYED ACTIVITY DISCHARGED TC PONDS AND DITCHES WTTHIN THE CHFMTSAL SEPARATION AREA CONTROL ZCNE THROUGH O/3E 1973

\section{DISPOSAL SITE}

VOLUME (L)

$$
\text { PU }
$$$$
\text { (GM) }
$$$$
\text { RETA }
$$

(CI)
RU-106

(CI)

$c s-137$
(cI)

Co-60 (CI)

$U$
(KGM)

$216-5-19$

1973 SIN

TOT SUM

TOTOECAY

$21 G-T-1$

1973 SUM

TOT SUM

TOTDECAY

$216-T-4$

1973 SUM

TOT EUY

TOTMFCAY

$216-U-9$

$216-1]-1$

1977 SUM

TOT SU:

TOTPECAY

222-S LA3 FOND. ELACED IN JSE FEQ 1952 AND IS STILL ACTIVE. APFROXIMATE CCORTINATES: N-3220, w-7291, N-31840, W-72650. REFERENCE DRAMINGS: $H-2-3476 ?$.

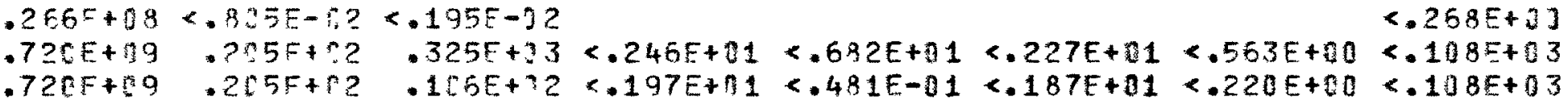

221-T (HFAD FNO) ITTCH. FLACED IN USF NOV 1944 ANC IS STILL ACTIVE. APFRCXTMATF COCR INATES: N-44455, W-7305: (HEAD) TO N-44570, W-7305. TO $\mathrm{N}-4495 \mathrm{E}, \mathrm{W}-7^{-} \mathrm{E} 2 \mathrm{2}$. (TEQMINUS). REFERFNCE DQAWINGS: H-2-5101, M-2964 SHT11.

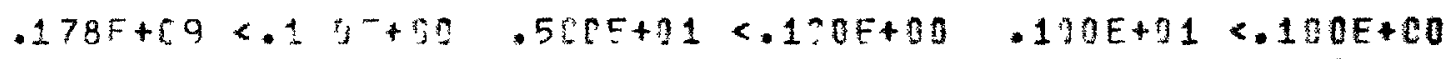

$T$ ELANT FOND. PLACFO IN ISE NOV 1944 AND IS STILL ACTIVF. APFROXIMATE CONPDINATFS: $N-44314, W-75630$ TO N-46150, W-75970 TO N-46150, W-77150 TO

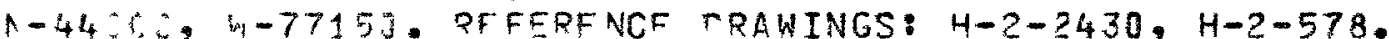

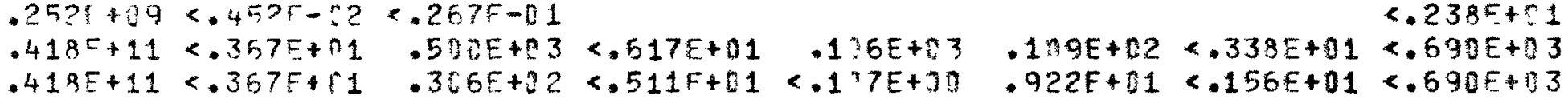

U FNNE OVFRFLS, FITCH. PLACEN IN USF MAR 1952 AND REMOVEC FRCM SERVICE An? 1954. AFFROXIMATF COJRDINATES: N-35750, $\mathrm{W}-73050$ (HEAC) TC N-359JI, W-77? ITERMTNUSI. DEFERENCE DRAWINRS: H-2-2433.

1 FCMD AID DITRHFS. TLACED IN USE MAR 1952 ANO JS STILL ACTIVE. APFRTYTMATE CONRTIMATES: M-37201, W-7901:N-35900, W-77000. "ZFFFAMCE MOAWINTS: H-2-243*, SK-2-1:83.

$.122 r+10<.795 r+01<.6255+00$

$<.172 E+91$ - $21 F+12$ - $111=+4$ $.375 F+34$ 
TABLF 1-A

SUMMARY OF CUFFFNT, TOTAL, DECAYFO ACTIVITY DISCHARGED WITHIN THE CHF ICAL SEPARATTON AREA COATROL 7ONE THROUGH G/30 1973

VOLUME FU TETA

DISFOSAL SITF

(L)

(GM)

META

(CI)

$S R-90$

(CI)

PU-116

(CI)

cS-137

(CI)

$(0-6)$

(CI)

41

(KGM)

$U-233$ (GM)

$216-v-11$

U FOND CUFRFLON FOND. OLACFD IN USE MAR 1952 ANE IS NDT USEO. 
SUMMARY OF CURRENT, TOTAL, DECAYFD ACTIVITY DISCHARGED TO CRIBS WITHIN THE CHEMTCAL CFPARATIONS APFA CONTROL TINE THROUGH 9/30 1973

\section{DISPOSAL SITE \\ $216-A-3$ \\ 1973 SUM \\ TOT SUM \\ TOTIECAY}

VCLUME DU

(C)
DETA

(CI)

\section{$5 Q-99$}

(CI)
RU -136

(CI)
CS -137
(CI)

\section{CO-60 \\ (CI)}

U

(KGM)
$U-233$

(GM)
$216-A-4$

1973 SUM

TOT SUY

TOTPECAY

21E-A-5

1973 SUM

TOT SUM

TOINECAY

$216-A-5$

1973 S114

TDT SUY

TOTNECAY
233-A SILITA GFL WASTES. PLACEN IN USE FFP 1956 AND IS INACTIVE.

DFPRAXTMATE CCOPTINATFS: N-40530, W-48540. RFFEOENCE DRAWINGSH-2-56050, $H-2-5.5 ; 40$.

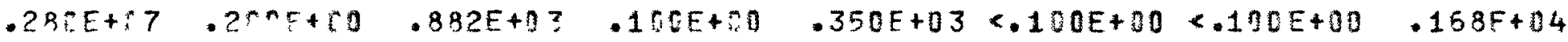

LAT AN7 STACK TRATN. PLAPEJ IN USE DEC 1955 AND REMOVED FROM SERVICE TEC 1959. APFPOXIMATE COORDINATES: N-39515, W-48158. REFERENCE DRAWINGS: $H-3-560=H-2-56049$.

$\begin{array}{llllllll}.521 E+i 7 & .14 n E+13 & .964 F+03 & .100 E+02 & .430 E+03 & .150 E+02 & .100 E+01 & .399 E+03 \\ .521 F+i 7 & .140 E+13 & .332 E+02 & .566 E+11 & .542 E-02 & .103 E+02 & .114 E+00 & .399 E+03\end{array}$

FPNCTSS CCND. PLACEO IN USE NOY 1955 ANJ PEMOVEO FROM SFRVICE NCV 1951. AFPROXIMATE COCRCINATFS: N-39510, W-48756. REFFRENCE ORAWINGS: H-2-56050, $\mathrm{H}-2-5 \mathrm{~F}^{\circ}+4 \mathrm{a}$.

$.163 F+10 \quad \cdot 55 F+-2 \quad .341 F+34 \quad .900 E+12 \quad \cdot 150 E+33 \quad \cdot 250 E+02 \quad \cdot 110 F+03 \quad \cdot 253 E+03$

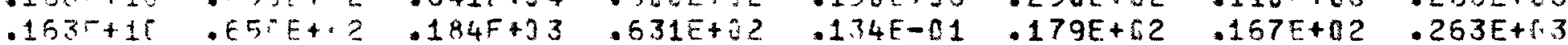

FUPFX CTFAM TOND. PLACED IU USE NOV 1955 AND REMOVED FROM SERVICE 1AN 1970. AFRSCXTAATF COOPJIIATES: N-3939O, W-47000. REFFRFNCE ORAWTMGS: $H-2-56 \% 1 F, H-2-5 E 715$.

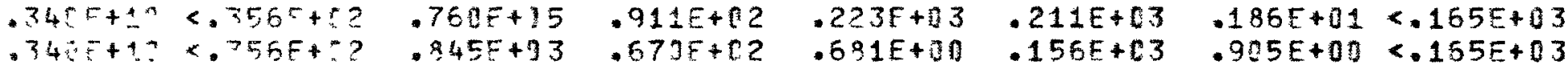


SUMMARY OF CLPFENT, TOTAL, \& DECAYEJ ACTTYITY DISCHARGED TC CRIBS WITHIN THE THFMICAL SFPARATTONS ARFA CONTPOL TONE THRCUGH $9 / 3$ : 1973

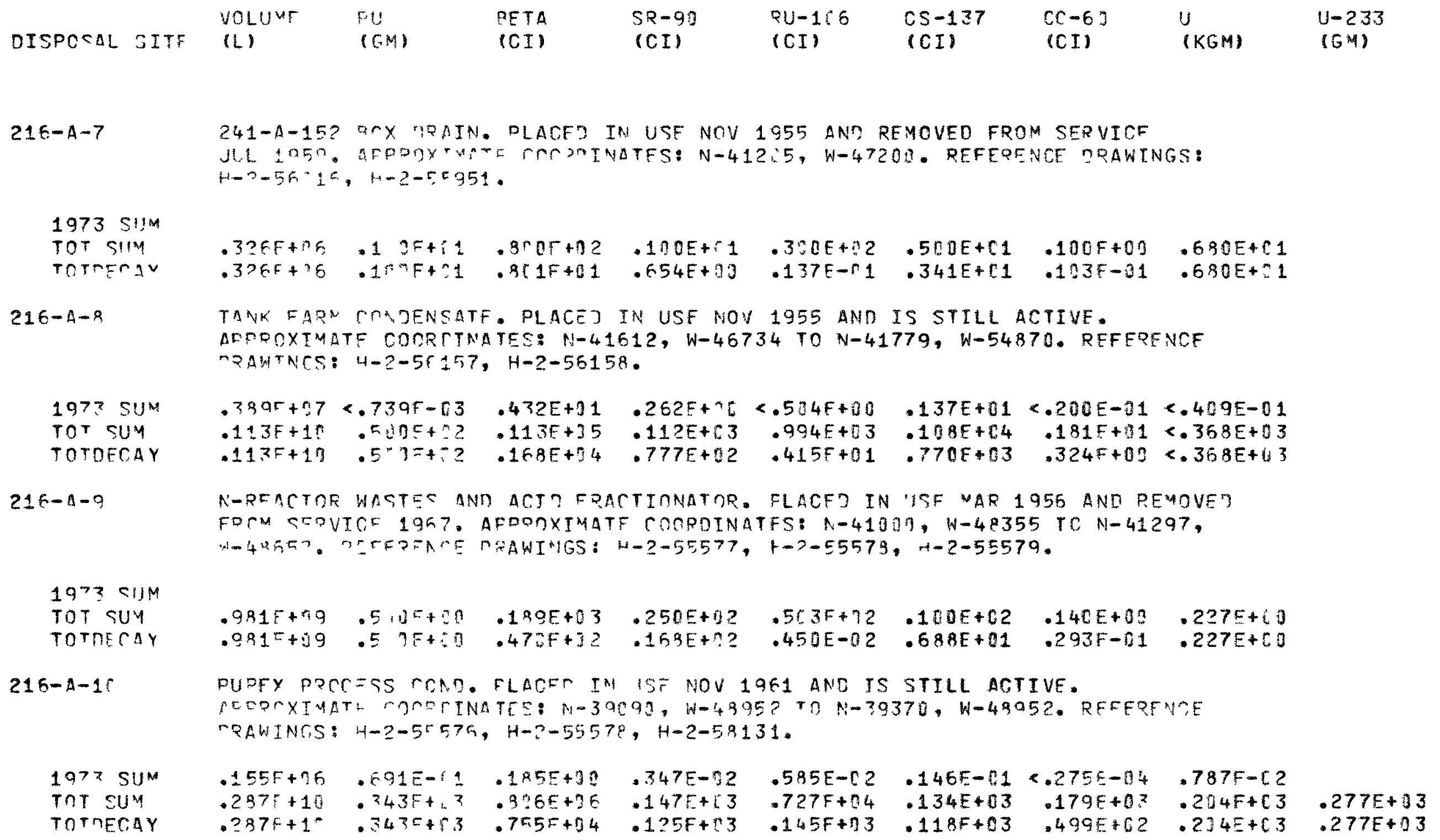

$.347 E-32$

$.147 \mathrm{~F}+\mathrm{L} 3$ $.287 F+1$

$.3435+73$

$.75 .5 F+34$

$.595 E-C 2$ $.727 F+04$ $.145 F+13$

$.146 F-81$ $.134 E+03$

. $118 F+03$

$2.2756-04$ $.179 E+0$ ? $.499 E+62$

$.787 F-C 2$ $.204 F+C 3$ $.234 E+C 3$ 
SUMMARY OF CURGENT, TOTAL, \& DFCAYFD ACTIVITY DISCHARGED TC CRIAS WITHIN THE THFNTCAL SEPAFATTCAS ARFA CONTROL ZONF THROUGH 9/30 1973

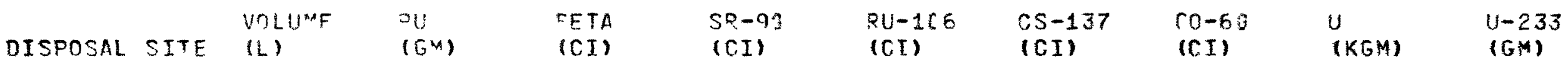

216-A-21 AMMONTA SCFUAZER ANR LAR WASTFS. PLACFO IN USE OCT 1957 AND REMOVFD FROM

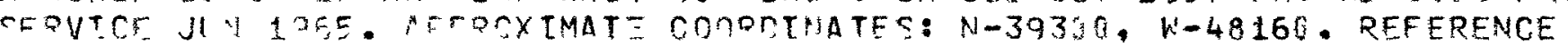
Donbitire: $4-2-57442, \mathrm{H}-2-57$ B 32 .

\begin{abstract}
1973 SUM TOT SIM
\end{abstract} TOTAECAY

$216-1-? 4$

$197^{2} \mathrm{SuM}$ T) बizy

tormecay

$21 E-A-27$

1973 SUM TOT SUM TOTPECAY

$216-A-29$

1973 sun TOT SUA

TOTDECAY

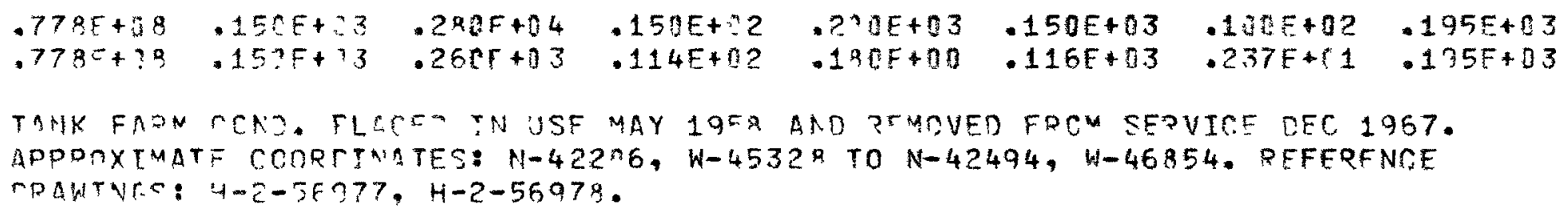

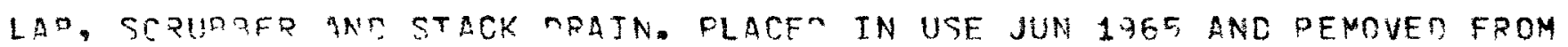

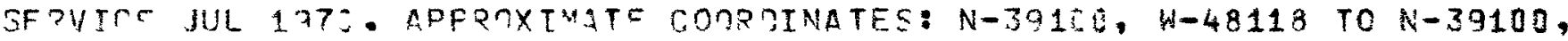
H-4.3312. DFFFRFNCF TPAWTNGS: $4-2-575: 9,1-2-57539$.

$.237 F+.8 \quad .955 E+112 \quad .258 E+04 \quad .413 F+02 \quad .632 E+32 \quad .526 E+82<.257 E+01 \quad .630 E+02$ $.232 f+19 \quad .055 F+[2 \quad .269 F+03 \quad .372 F+32 \quad .171 E+11 \quad .479 E+02<.151 E+31 \quad .680 E+02$

$-1685+93$ $168 E+03$
2R3-A ANT UNH TANK WASTES. PLACED IN USE DFC 1958 AND RENDVED FRCM SERVICF

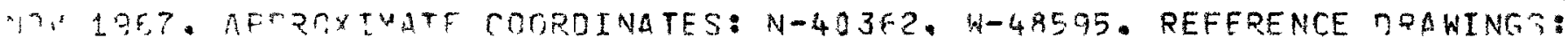
$H-2-57517$.

\section{$.7505+05$}

$.3325+.5$ 
SUMMAPY TF CUREFNT, TOTAL, \& DECAYED ACTIVITY DISCHARGED TC CRIBS WITHIN THE THEMTSAL SFFAFATTONS AREA CONTROL TONF THRCUGH $9 / 301973$

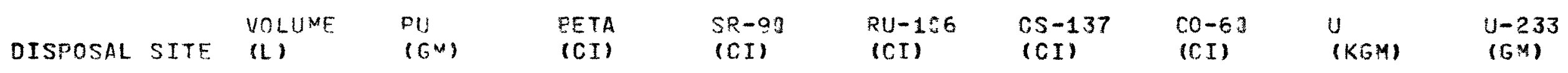

$216-A-3$

$$
\begin{aligned}
& 1973 \text { SUM } \\
& \text { TOT SUM } \\
& \text { TOTrECAY }
\end{aligned}
$$

$21 E-A-3 F R$

1973 SUM TOT SUM TOTRECAY

$216-A-27$

$216-A-39$

$216-3-5$

1973 SUM TOT SII TOTRECAY

CUPFX STFAM CONF ELACFO IN USF JAN 1961 AND IS STTLL ACTTVE. AFFROXTMATE

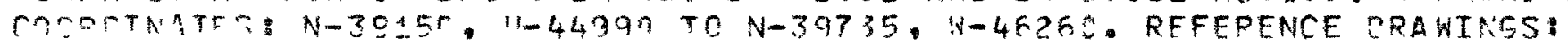
$H-2-577 ?, H-2-57719$.

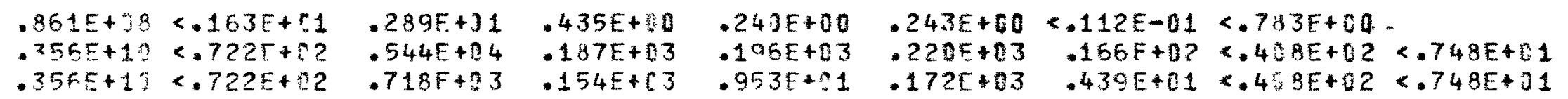

AMMRAIA SCPUABER WASTES. DLACFO TN USE MAP 1966 ANJ IS INACTIVE.

APPROXIMATE CONPCINATES: N-33500, W-48525 TO N-39000, W-48525. REFERFNCE ORAWTNGS: $4-2-50,875, H-2-59129$.

$.945+.0 .177 E+13 \quad .475 E+05 \quad .546 E+C 3 \quad .653 E+34<.556 E+C 3<.514 E+12$ $0.94: 5+920.1775+13$ $.631 \mathrm{~F}+34$ $5=C E+13$ $.121 E+04<.516 E+03<.344 E+02$

$119 E+13$ $.119 E+03$

-TFAM COMN. NOT JSFT. AOPROXTMATF COOROINATFS: N-3995E, W-45816 TO N-40157, W-45440. RFFEQENCE RRAWINGS: H-2-57719, H-2-62876, H-2-57729, $1-2-52977$

FPOCESS COND. NCT USEN. APPPOXTMATE SOORDINATFS: N-38971, W-49352 TO $\mathrm{N}-30471, \mathrm{~W}-49352$. REFEPENCE DRANINGS: H-2-62876, H-2-62875, $\mathrm{H}-2-62877$.

2?:-P AND 5-6 WASTFS. DLACED IN USE APR 1945 AND REMOVED FROM SERVICE COT 1947. APPQOXTMATF CNORIINATFS: N-43490, W-52855. REFERFNCE DRAWINGS $+-?-1\} ? 1, H-2-1123$.

$\begin{array}{llllll}.3765+9 & .428 E+4 & .380 E+34 & .756 E+? 2 & .153 F+03 & .907 E+62 \\ .35 F E+9 & .429 F+44 & .161 E+03 & .387 E+72 & .128 E-05 & .431 E+12\end{array}$


SUMNAPY AF CURFENT, TOTAL, $\triangle$ DECAYFD ACTIVITY NISCHARGFO TC CRIBS WTIHEN THE CHEMICAL SFDARATIOAS AREA CONTROL TOVE THROUGH $9 / 301973$

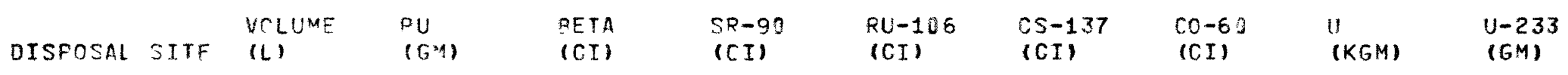

$216-8-10 P$ 1973 SUM
TOT SUM TOTSFCAY $216-9-11$

1973 sum TOT SUM TOTSFCAY $216-9-12$

1973 SIM TOT SUM TOTREAY

$216-3-14$

- PLANT R-i3 WASTE. PLACED IN USE JUN 1969 AND IS STILL ACTIVE. APFRRXIMATE COCRTINATES: N-43195, h-53943. REFERENGE DRAWINGS: H-2-32522, $\mu-2-1549$.

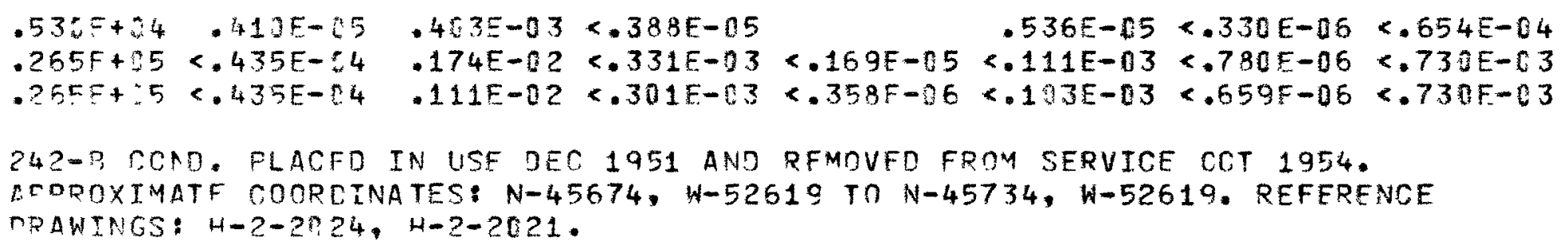

242-? SCND. PLACFD IN USE DEC 1951 AND RFMDVFD FROM SERVICE CCT 1954. ICOROXIMATF COORCINATES: N-45674, W-52619 TN N-45734, W-52619. REFERENCE RRAWINGS: $H-2-2 n 24, H-2-2021$.

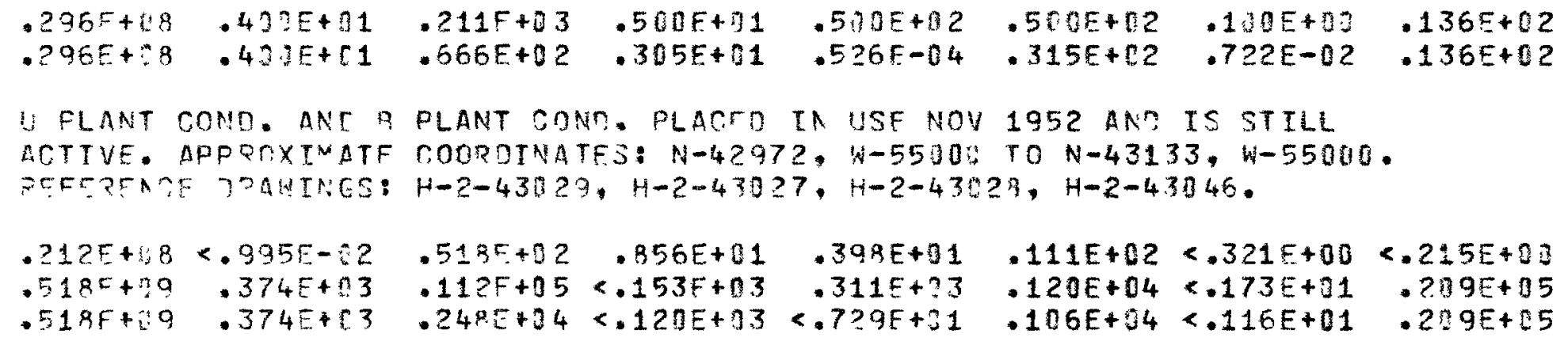

U PLANT CCAUFNGES WASTE. PLACES IN USE JAN 1956 AND REMOVEO FROM SEPVICE FF? 1955. $4 F F R$ IXTMATE COORDINATES: N-35855, W-53309. REFERENCE DRAWINGS: $H-2-29: 3, \mathrm{H}-2-29: 7$.

19735119 TOT SUM TOTRF.CAY

$.8715+37 \quad .252 E+22 \quad .140 E+06, .45$ $.371 E+: 7 \quad .25:+2 \quad .872 F+03 \quad .252 E+3$
$.595 F+15$ $.431 F+10$
$.2505+63$ $.168 E+13$
$.500 E+01 \cdot 218 E+03$ $.514 F+03 \quad .218 E+33$ 
SUAMARY DE CUREENT TOTAL, DECAYED ACTIVITY DISCHARGEO TO CRIBS WITHIN THE CHEATCAL SEPARATTONS AREA CONTROL ZOHE THROUGH $9 / 301973$

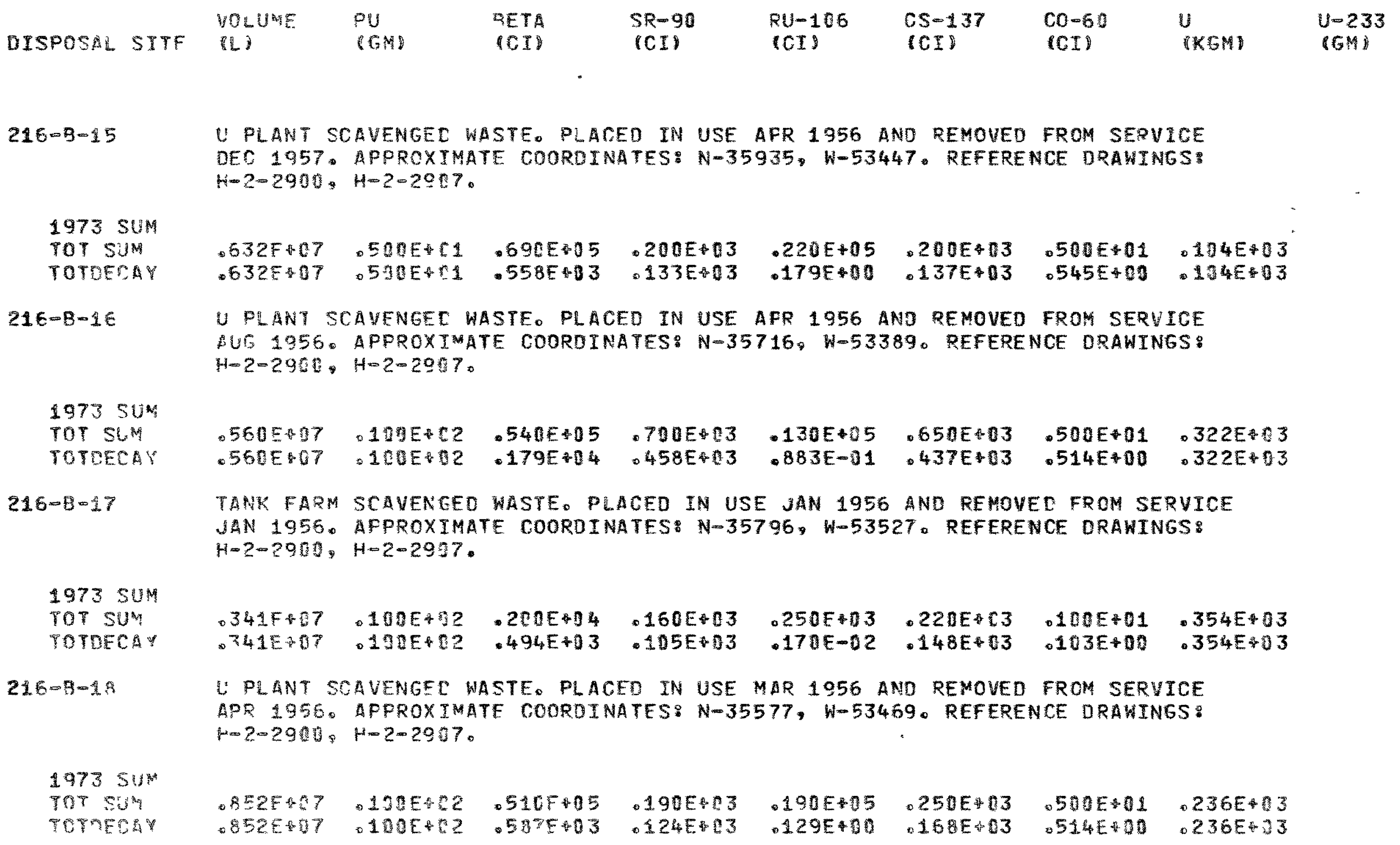




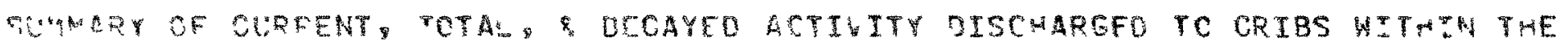
CHEWTAL EEDARATTOAS AREA CCATROL TONE THROUGH Q/301973

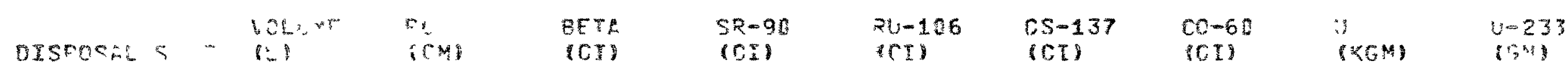

$216-9-1=$

1973. SUM TOT SUP

TOTHFis:

$216-8=\cdots$

19735 $\operatorname{TnT} 33^{*}$

POTESA 1

$216-9-44$

$19738)^{3}$ Tot S.M

TPTEET

$216-8-45$

1977 w

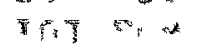

hing

6 FLAIT ASO TAK FARM SCAVENGES WASTE. PLACEC IN USE FEB 1957 AND REMOUE: FKOH SEPUICE CCT 1957. APPROXIMATE COOROTMATES: N-35657. W-53617. REFEPENCF 2RA WIACS: H-2-290, $\mathrm{H}-2-297$.

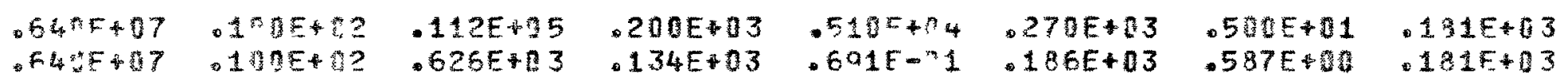

$\therefore$ P. RNT SCAVENGE WASTE. PLACED IN USE NCU 1954 ANA REMOVEO FROM SERVTSE NAV 1954. APPQOXIMATF CONROINATES: N-46332, W-53355. REFERENCE BRAWTGS: $1-2-2533,4-2-25 P^{2} 5$

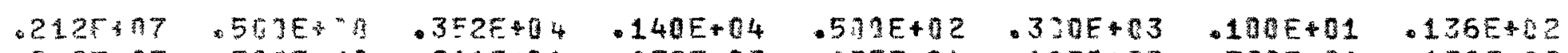
$.212 F+07$.5.

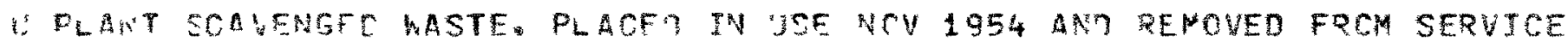
MR 1955. APPRCX IAATE COORJT NATES: N-46417, h-53355. REFERENCE DRANINTS: $1-2=25 n \pi ;=2-2605$.

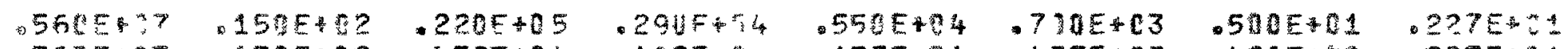

U DLAPT SCAUEMEEC WASTE. PLACED IN USE APR 1955 ANT RFMCVED FROM SFQVIPE SW 1955 . AFFPOXIMATE COBRUTNATES: -46502 , W-53355. CEFERENCE DRAIINGS: $f=2-2643,4-2=26 \neq 5$.

\begin{tabular}{|c|c|c|c|c|}
\hline 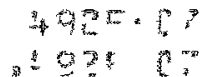 & 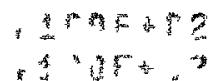 & $\begin{array}{l}.530 E+15 \\
.549 E+14\end{array}$ & $\begin{array}{l}.280 E+E_{14} \\
0179 E+14\end{array}$ & $\begin{array}{r}.170 E+15 \\
.579 E-11\end{array}$ \\
\hline
\end{tabular}


SUMAARY DF GUPRENT, TOTAL, DECAYED ACTIVITY DISCHARGED TC CRIBS WITHIN THE CHFMICAL SEFAPATIONS AREA GONTROL ZONE THROUGH $9 / 301973$

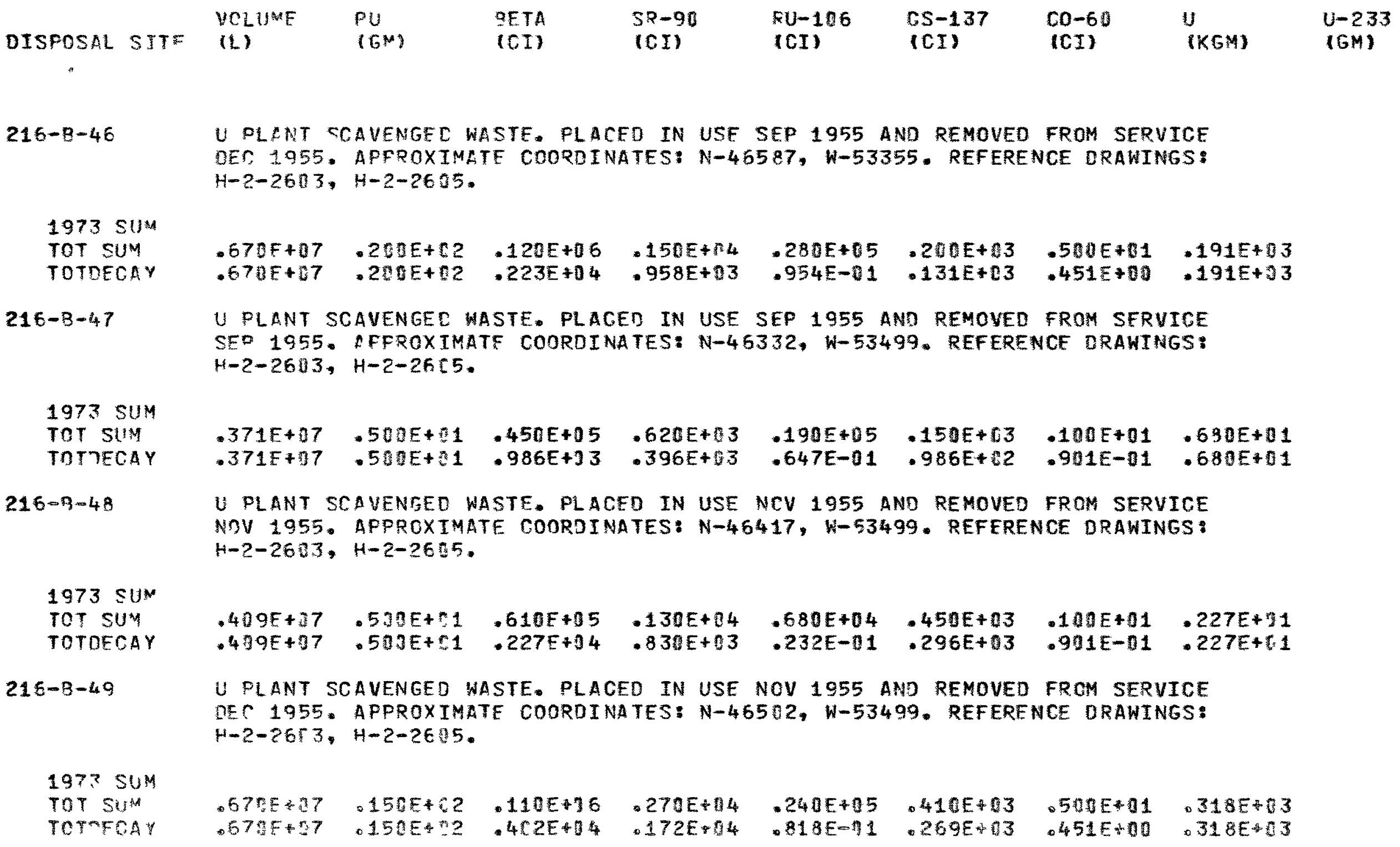


SUMMARY OF CUPRENT, TOTAL, DECAYED ACTIVITY DISCHARGEO TC CRIBS WITHIN THE CHEMICAL SEFARATIONS AREA CONTROL ZONE THROUGH 9/30 1973

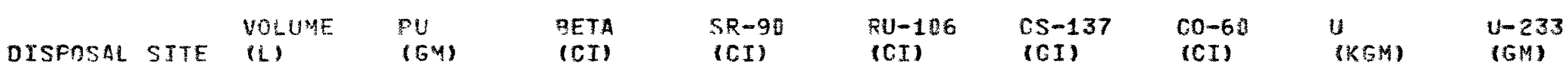

$216-9-5+3$

$1973 \mathrm{SuM}$ TOT SUM TOTDFCAY

$216-9-55$

1973 SUM TOT SUM

TOTOECAY

$216-9-57$

$1973 \mathrm{SHM}$ TOT SU: TOTDECAY

$216-C-1$

1973 SUM

TOT SUM

TOTDECAY

ITS-1 WASTE PLACED IN USE JAN 1965 AND IS STILL ACTIVE. APPROXIMATE CCORDINATES: $N-465.87, W=53499$. REFERENCE DRAWINGS: $H-2-2603, H-2-2605$.

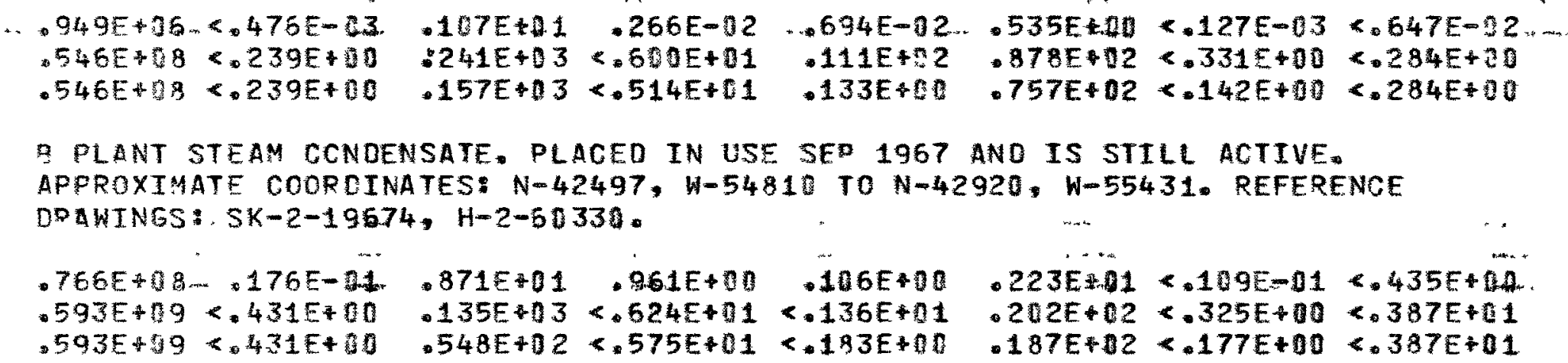

9 PLANT STEAM CCNDENSATE. PLACED IN USE SE 1967 AND IS STILL ACTIVE. APPROXIMATE COORCINATES: N-42497, $W-54810$ TO N-42920, W-55431. REFERENCE DOAWTNGS: $5 K-2-19674, H-2-60330$.

\begin{tabular}{|c|c|c|c|c|c|}
\hline $\begin{array}{l}766 E+08-176 \\
593 E+09<.431 \\
593 E+19<.431\end{array}$ & $\begin{array}{l}.871 E+01 \\
.135 E+03 \\
.548 E+02\end{array}$ & $\begin{array}{r}.961 E+00 \\
<.524 E+11 \\
\times .575 E+01\end{array}$ & $\begin{array}{r}106 E+00 \\
<.136 E+01 \\
<.193 E+00\end{array}$ & $\begin{array}{l}E+12 \\
E+62 \\
E+12\end{array}$ & $\begin{array}{l}E-01<.435 E+0 A \\
E+00<.387 E+1 \\
E+00<.387 E+01\end{array}$ \\
\hline
\end{tabular}

ITS-2 WASTE. PLACED IN USE FEB 1968 AND IS STILL ACTIVE APPROXIMATE COSRIINATES: $N-4 E 160, W-53775$ TO N-46360, W-53775. REFERENCE DRAWINGS $H-2-6240$.

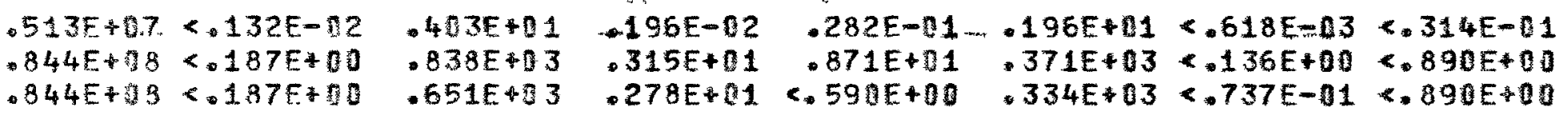

2.1-C PRCCESS CONDENSATE. PLACED IN USE JAN 1953 AND REMCVED FRON SERVICE JUN 1957. AFFPDXIMATE COORDINATES: $N-42069, N-50235$. REFERENCE DRAWINGS $\mathrm{H}-2-4037, \mathrm{H}-2-32523$.

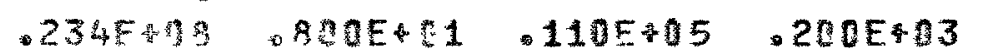

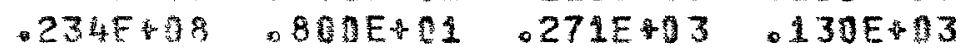

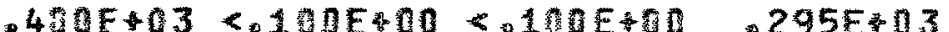
$.234 E-02<.672 E-01<.103 E-01$. $295 E+3$ 
SUAMARY OF CURRENT, TOTAL, DECAYEO ACTIVITY DTSCHARGED TC CRTSS WITHIN THE CHAMTCAL SEPARATTONS AREA CONTROL ZONE THROUGH $9 / 301973$

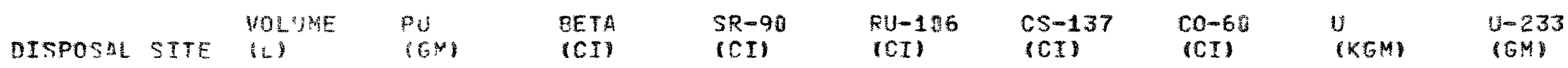

$216-c-3$

1973 S:3** TOT SQMA

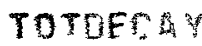

$216-\mathrm{C}-\mathrm{S}$

rot sul

TOTHECA

$216=\mathrm{C}-7$

1973 SUM

TOT SUM

TOTOECAY

$216-6-16$

271-R CHEMICAL WASTE. FLACED IN USE JAN 1953 ANO REMOVED FRON SERVTCE MAP 1954. $\triangle F P R C X I A A T E$ CODRDINATES: N-42055, W-50390, REFERENCE DRAWINGS: $H-2-43, H-2-32523$,

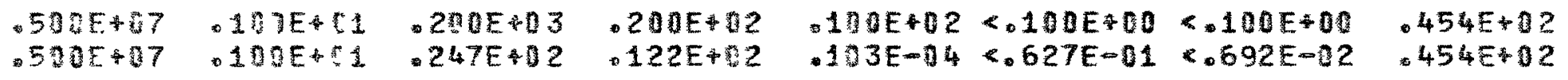

201-C FIGH SALT WASTE FLACED IN USE MAR 1955 AND REMOVEC FRCM SERUTCE JUN 1955. AFFRCXIMATE CODRDINATES: N-42030, W-50360. REFERENCE DRAWTNGS: $\mathrm{H}-2-4010, \mathrm{H}-2-4425$

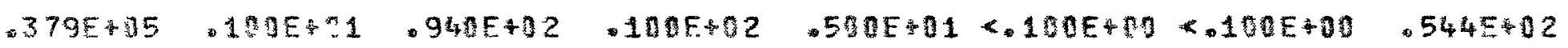

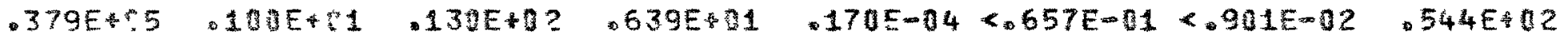

209-F CRITICAL MASS LAB. PLACEN IN USE JAN 196ः AND IS STILL ACTIVE. AFPROXIMATE COOREINATES: $\mathrm{N}-42000, W=50672$. REFFRENCE DRAWINGS: $\mathrm{H}=2-44336$, $H-2-32523$.

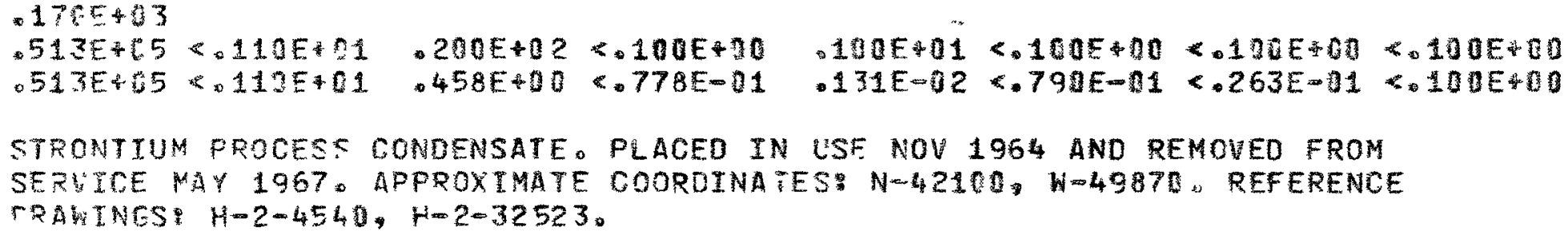


SUMMARY AF CURPENT, TOTAL, \& DECAYFD ACTIVITY DISCHARGED TC CRIBS WITHIN THE CHEMICAL SEPARATIONS AREA CONTROL ZONE THROUGH 9/30 1973

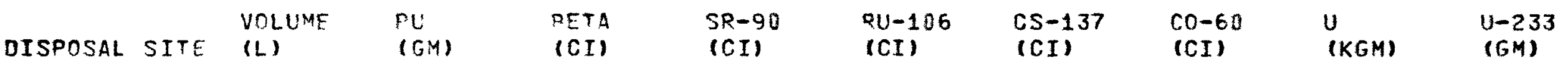

$216-N-2$

\section{SUM} TOT SUM

TOTIFCAY

$216-N-3$

1973 SUM TOT SUM

TOTDECAY

$216-N-5$

1973 SUN TOT SUM TOREECAY

$216-N-7$

1973 SUM TOT SUM TOTDECAY
212-N TASTN ANT SLUDGE CLEAN-OUT. PLACFD IN USE MAR 1947 AND REMOVED FROM SFRVICE AND 1947. APFRCXIMATE CCORDINATES: N-55487, W-65788. REFERENCE ERAWTRES: $H-2-32524$.

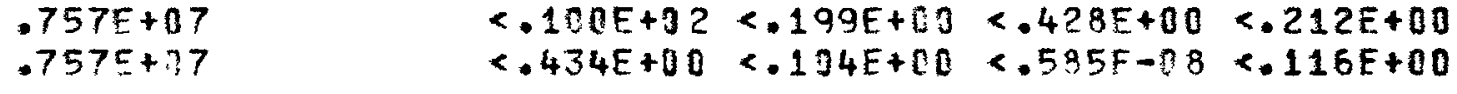

212-N BASIN AND SLUDGE CLEAN-OUT. PLACEO IN USE MAY 1952 AND REMOVEO FROM SFRVICE JUN 1952. AFFROXIMATE COORDINATES: N-55455, W-65839. REFERENCE TRAWING: $H-2-32524$.

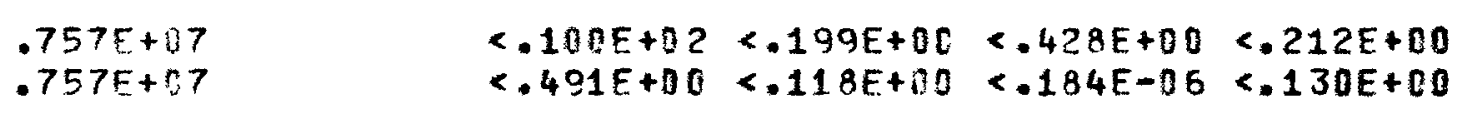

$<.100 E+02<.199 E+30<.428 E+00<.212 E+00$

$.757 E+07$

$<.491 F+00<.118 E+00<.184 F-06<.130 E+00$ 
SUMMARY DF CURRENT, TOTAL, \& DECAYFD ACTIVITY DISCHARGED TC CRIBS WITHIN THE CHFMICAL SEPARATIONS AREA CONTROL ZONE THROUGH $9 / 301973$

DISPOSAL SITE

$216-51 \& 2$

$1973 \mathrm{SUM}$ TOT SUM TOTIFRAY

$216-s-3$

1973 SUM TOT SUM TOTDECAY

$216-5-5$

$1973 \mathrm{SUM}$ TOT SUM TOTOFCAY

$216-5-5$

1973 SUM TOT SUY TOTRFCAY
VOLUME (L)

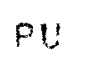

(GM)

RETA

$S R-90$

(CI)
२U-1:6
(CI)

$00-60$

(CI)

$U$

(KGM)
$U-233$

(GM)

REDOX PROCESS CCNDENSATE. DLACFD IN USE JAN 1952 AND REMOVED FROM SERVICE JMN 1955. ARPQAXTAATF COORTINATES: N-35419, W-75177 TO N-35444, W-75214. REFERENCF DRAWINCC: H-2-1313, H-2-1774, H-2-1776.

$\begin{array}{llllllll}.160 E+09 & .120 F+04 & .75 C E+06 & .300 E+04 & .300 E+04 & .250 E+04 & .100 E+02 & .227 E+04 \\ .160 E+r 9 & 1235+.14 & .754 E+04 & .180 E+04 & .767 E-02 & .162 E+04 & .843 E+30 & .227 E+04\end{array}$

241-S TK-101 ANT 104 CONHENSATF. PLACEO IN USE AUG 1953 AND RFYOVED FROM SERVICE AUG 195F. AFPROXIMATE COOROINATES: N-36000, W-75500. REFERENCE RRAWINFS: $\mathrm{H}-2-30574, \mathrm{H}-2-1813$.

$\begin{array}{llllllll}.420 F+07 & .507 F+E & .270 E+03 & .100 E+01 & .550 E+02 & .530 E+02 & .100 E+01 & .381 E+00 \\ .420 E+07 & .500 E+C B & .637 E+02 & .629 E+70 & .135 E-03 & .324 E+02 & .836 E-01 & .381 E+00\end{array}$

STFAY CONOFNSATE. PLACED IN USE MAR 1954 AND PEMOVEO FRON SERVICE MAR 1957. ACORCXIMATE COORDINATES: N-32740, W-77000. REFERENCE DRAWINGS: H-?-E963, H-2-50 5 .

$\begin{array}{llllllll}.410 E+10 & .580 E+: 3 & .960 F+03 & .130 E+03 & .3010+02 & .600 E+02<.100 E+00 & .272 E+C 3 \\ .410 F+10 & .580 E+.3 & .240 F+03 & .822 E+02 & .894 E-04 & .390 E+12 & <.790 E-02 & .272 E+03\end{array}$

RETOX STEA CONDENSATE. PLACED IN USE NOV 1954 AND IS INACTIVE. APPROXIMATF COORCINATES: N-33250, W-77850. REFERENCE DRAWINGS: H-2-2594, $4-2-2595$.
$.447 F+10$
$.473 E+i 3$
$.525 E+04$
$.412 E+33$
$.133 E+03$
$.212 E+03<.294 E+01<.272 E+03$ $.447 E+13$
$.473 E+03$
$.979 E+33$
$.310 E+03$
$.736 E+0 \%$
$.170 E+03<.130 E+01<.272 E+03$ 
SUMMARY OF CURRFNT, TOTAL, \& OECAYED ACTIVITY DTSCHARGED TC CRIBS WITHIN THE CHEMICAL SEPARATIONS AREA CONTROL ZONE THROUGH $9 / 301973$

DISPOSAL SI
$\begin{aligned} & 215-5-7 \\ & 1973 \text { SUM } \\ & \text { TOT SUM }\end{aligned}$

TOTRECAY

$216-5-9$

1973 SUM TOT SUM

TOTDECAY

$216-5-18$

1973 SUM

TOT SUM

TOTDECAY

$216-5-20$

1973 SUM TOT SUY TOTDECAY

$\begin{array}{lllllllll}\text { VOLUME } & \text { PU } & \text { RETA } & S R-9 R & R U-1 C 6 & C S-137 & C O-6) & U & U-233 \\ (L) & \text { (GM) } & \text { (CI) } & \text { (CI) } & \text { (CI) } & \text { (CI) } & \text { (CI) } & \text { (KGM) } & \text { (GM) }\end{array}$

PROCESS COND. PLACED IN USE JAN 1956 ANR RFMOVEO FROM SERVICE JUL 1965. APPROXIYATE COORETNATES: N-35152, W-74510. REFERENCE ORAWINGS: H-2-30135.

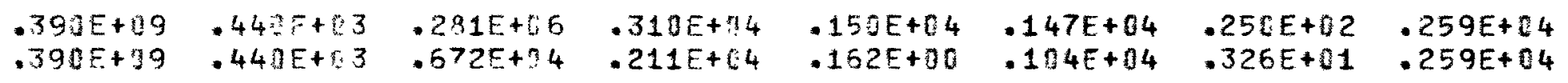

PROCESS COND. FLACED IN USE JUL 1965 AND REMOVED FROM SERVICE JAN 1969. APPROXIMATE CONREINATES: N-36150, W-7455. REFERENCE DRAWINGS: H-2-32363, $H-2-32362$.

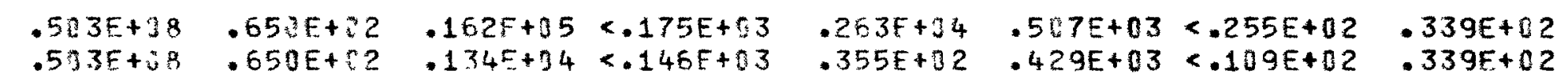


SUMMARY DF CURRENT, TOTAL, DFCAYED ACTIVITY OISCHARGED TO CRIBS WITHIN THE RHFNICAL SEPARATIONS AREA CONTROL TONE THROUGH $9 / 301973$

\begin{abstract}
DISPOSAL SITE
$216-5-21$

1973 SUA TOT SUM
\end{abstract}

VOLUME PU

(L)

(GM)

TETA

(CI)

$S R-90$

(CI)

RU-1C6

(CI)
CS-137
(CI)

$\mathrm{CO}-60$
(CI)

$U$
(KGM)

$U-233$

(GM)

241-SX COMJ. PLACED IN USE NCV 1954 ANO IS INACTIVE. APPROXIMATE

CNCRTINATFS: N-3E923, ;-75403. REFERENCE RPAMINSS: H-2-39549, H-2-39574. TOTDECAY

$216-5-22$

1973 SUM T2T SUY TOTRECAY

$216-9-23$

$197^{2} \mathrm{SU}^{\mathrm{M}}$ TOT SUM

TOTIECAY

$216-T-3$

1973 SUM TOT $\operatorname{tun}^{4}$ TOTIECAY

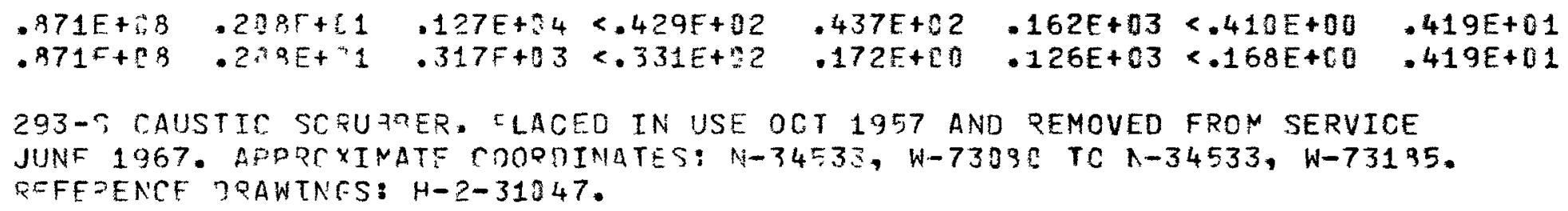

224-T, 5 AND 6 WASTFS. PLACED IN USE JUN 1945 AND REMOVED FRCM SERVICE AUG 1946. APPROXIMATE COORIINATES: N-43335, W-74250. REFERENCE DRAWINGS: $\mathrm{H}-2-353, \mathrm{H}-2-951$.

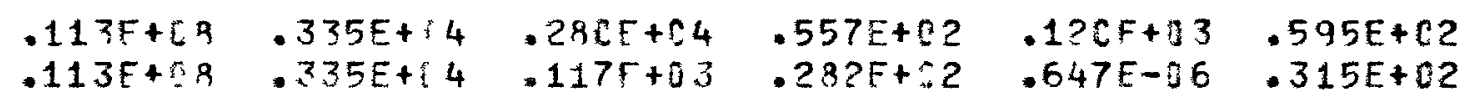


SUMMARY OF CUPRENT, TOTAL, DFCAYFD ACTIVITY DISCHARGED TO CRIBS WITHIN THE CHFMICAL STPAPATIONS AREA CONTROL ZONE THRCUGH $9 / 301973$

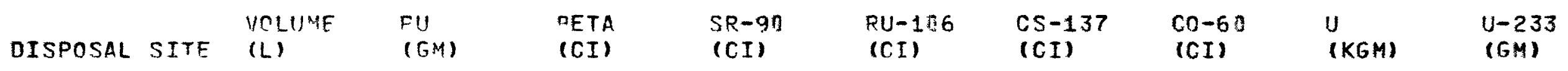

$216-T-6$

1973 SUM TOT SUM

TOTOECAY

$21 E-T-7$

1973 SUM TOT SUM TITDECAY

$21 \epsilon-T-8$

1973 SUM TOT SUM TOTOECAY

$216-T-19$

1973 SUM TOT SUY TOTNECAY
P24-T 5 AMN 6 WASTES. PLACED IN USE AUS 1945 AND REMOVED FROM SERVICE CCT 1947. BFPPOXINATE COORTINATES: N-43315, W-74453 TO N-43315, W-74525. PFFFDENCF DRANINCS: H-2-353, H-2-951.

$\begin{array}{llllllll}.450 F+8 & .390 E+03 & .180 E+05 & .360 E+03 & .670 E+03 & .320 E+03 & .5 E 0 E+71 & .227 E+02 \\ .450 F+09 & .393 E+13 & .505 E+03 & .188 E+33 & .752 F-15 & .163 E+03 & .153 E+07 & .227 F+02\end{array}$

224-T 5-E AND SECCNC CYCLF. PLACED IN USE APR 1943 AND REMOVFO FROM SERVICE NOV 1955. APFROXTMATF COORDINATES: N-43285, W-76000 TO N-43285, W-7F356. RFFERENCF DRAKINGS: H-2-57R, HW-72192 \#1. $\begin{array}{llllllll}.110 E+99 & .130 F+23 & .310 E+\eta 4 & .6 C 0 E+02 & .100 E+03 & .500 E+02 & .160 E+01 & .910 E+01 \\ .110 E+39 & .135 E+n 3 & .135 E+33 & .364 E+02 & .127 E-03 & .313 E+02 & .715 E-01 & .910 E+01\end{array}$

222-T LAO WASTES. PLACFD IN USE MAY 1950 AND REMOVED FROM SEFVICE SEP 1951. AFPRNXIMATF CONROINATFS: N-43545, H-7295]. REFERENCE DRAWINGS: H-2-3E 3 .

$.580 E+06$.50F+21 .1CEF+03 . 100E+R1 .500E+01 . 100E+01<.100E+00 .450E+11

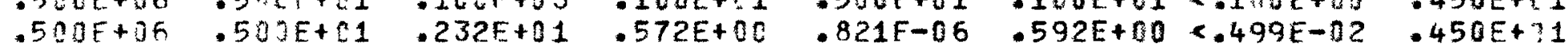

224-T, 242-T, 5-E, SFCMII CYYCLF WASTE EVAP. PLACED IN USE SFF 1951 AND IS STILL ACTTVE. AFFROXIMATE COORDINATFS: N-41270, W-756CO. REFERFNCE TRAWINGS: $H-P-3: F, H-2-3019$.

\begin{tabular}{|c|c|c|c|c|c|c|c|}
\hline $\begin{array}{l}.158 F+38 \\
.419 F+69 \\
.419 F+9\end{array}$ & $\begin{array}{l}.282 F-12 \\
.143 E+12 \\
.143 F+12\end{array}$ & $\begin{array}{l}.423 E+11 \\
.454 E+.14 \\
.579 E+13\end{array}$ & $\begin{array}{l}.460 F+110 \\
.620 E+12 \\
.412 E+02\end{array}$ & $\begin{array}{r}.142 F+00 \\
.100 E+04 \\
<.283 E+00\end{array}$ & $\begin{array}{l}.148 E+01 \\
.370 E+03 \\
.256 E+03\end{array}$ & $\begin{array}{r}<.133 E-02 \\
.105 E+01 \\
<.13 \hat{A} E+00\end{array}$ & $\begin{array}{r}147 E+1 \\
\text { ? } 945 E+01\end{array}$ \\
\hline
\end{tabular}


SUMMARY OF CURRENT, TOTAL, \& DECAYFO ACTIVITY DISCHARGED TC CRIBS WITHIN THE CHEMTSAL SEPARATTOAS AREA CONTROL 7ONE THROUGH $9 / 301973$

\begin{tabular}{|c|c|c|c|c|c|c|c|c|c|}
\hline DISPOSAL SITE & $\begin{array}{l}\text { VOLUME } \\
\text { (L) }\end{array}$ & $\begin{array}{l}P U \\
(G M)\end{array}$ & $\begin{array}{l}\text { PETA } \\
\text { (CI) }\end{array}$ & $\begin{array}{l}S R-9 D \\
\text { (CI) }\end{array}$ & $\begin{array}{l}R U-1: 6 \\
(C I)\end{array}$ & $\begin{array}{l}C S-137 \\
(C I)\end{array}$ & $\begin{array}{l}(0-60) \\
\text { (CI) }\end{array}$ & $\begin{array}{l}U \\
\text { (KGM) }\end{array}$ & $\begin{array}{l}U-233 \\
(G M)\end{array}$ \\
\hline
\end{tabular}

$216-T-20$

197.7 SUM

TOT SUM

TOTIECAY

$216-T-2 \epsilon$

1973 SUM

TOT SUY

TOTDECAY

$215-T-27$

1973 SUM

TOT SUM

TOTMECAY

$216-T-? 8$

1973 CUM

TOT SUY

TOTIECAY
241-TX-155 DIYGOSICN BOX. PLACED IN USE NOV 1952 AND REMOVED FROM SERVICE NDV 1952. AFEQ?XTMATF CONOITNATFS: N-41390, W-74723. REFERENCE DRAWINGS: $H-2-4451$ P SHT -3 .
$1995+1.5$
$.500 E+02$
$.994 E+10$
$.214 E+01$
$.106 F+B 1$
$.199 F+15$
$.590 E+60$
$.921 F-06 \quad .651 E+00$
$.500 E+C 1$ $.500+01$

SCAVFNGE WASTES. PLACED IN USF AUG 1955 AND PEMOVED FROM SERVICE NOV 1956. AFPROXIMATE COORDINATES: N-42275, W-75330. REFERENCE DRAWINGS: $\mu-2-2733, \mu-2-2735$.
$.1275+9$
$.597 E+82$
$.290 E+05$
$.670 E+103$
$.260 F+04$
$993 E-62,110 E+E 3$
$.100 E+01$
$.150 E+[3$
$.120 F+38 \quad .590 E+02 \quad \cdot 1 C 9 F+04$
$428 E+3$
$112 E+03$
$.952 E-01$
$.150 E+03$

RFOONTAMINATION, SCAVENGED WASTE 300 AREA. PLACEO IN USE SEP 1965 AND REYOVED FROM SERVICE NOV 1965. APPROXIMATE COORDINATES: N-42360, W-75330. GFFFPENTE DOANINCS: H-2-2733, H-Z-2735.

$\begin{array}{llllllll}.719 F+[7 & .130 E+02 & .36 j E+04 & .140 E+03 & .150 E+24 & .100 E+03 & .100 E+01 & .726 E+[1 \\ .719 E+07 & .137=+.2 & .309 E+03 & .114 E+[3 & .546 E+1 & .827 E+02 & .337 E+00 & .726 E+31\end{array}$

CECONTAMINATION AND 330 ARFA. PLACED IN USE FEP 1960 AND REMOVED FROM SERVICF DER 19EE. APPROXIMATF COORDINATES: N-42445, W-75330. REFERENCE ORAWINES: H-?-27:3, H-2-2735.

$\begin{array}{llllllll}.423 F+39 & .750 E+.2 & .585 E+35 & .200 E+[3 & .150 E+04 & .350 E+03 & .530 E+01 & .391 E+03 \\ .423 E+0.00 & .700 E+2 & .139 E+04 & .161 E+03 & .242 E+01 & .285 E+03 & .160 E+01 & .391 E+03\end{array}$


SUMMAY OF CUEFENT, TOTAL, DFCAYED ACTIVITY DISCHARGED TC CRIES WTTHIN THE CHE TOAL MEFAEATTOHS AREA CONTQOL ZONE THROUGH 9/3H 1973

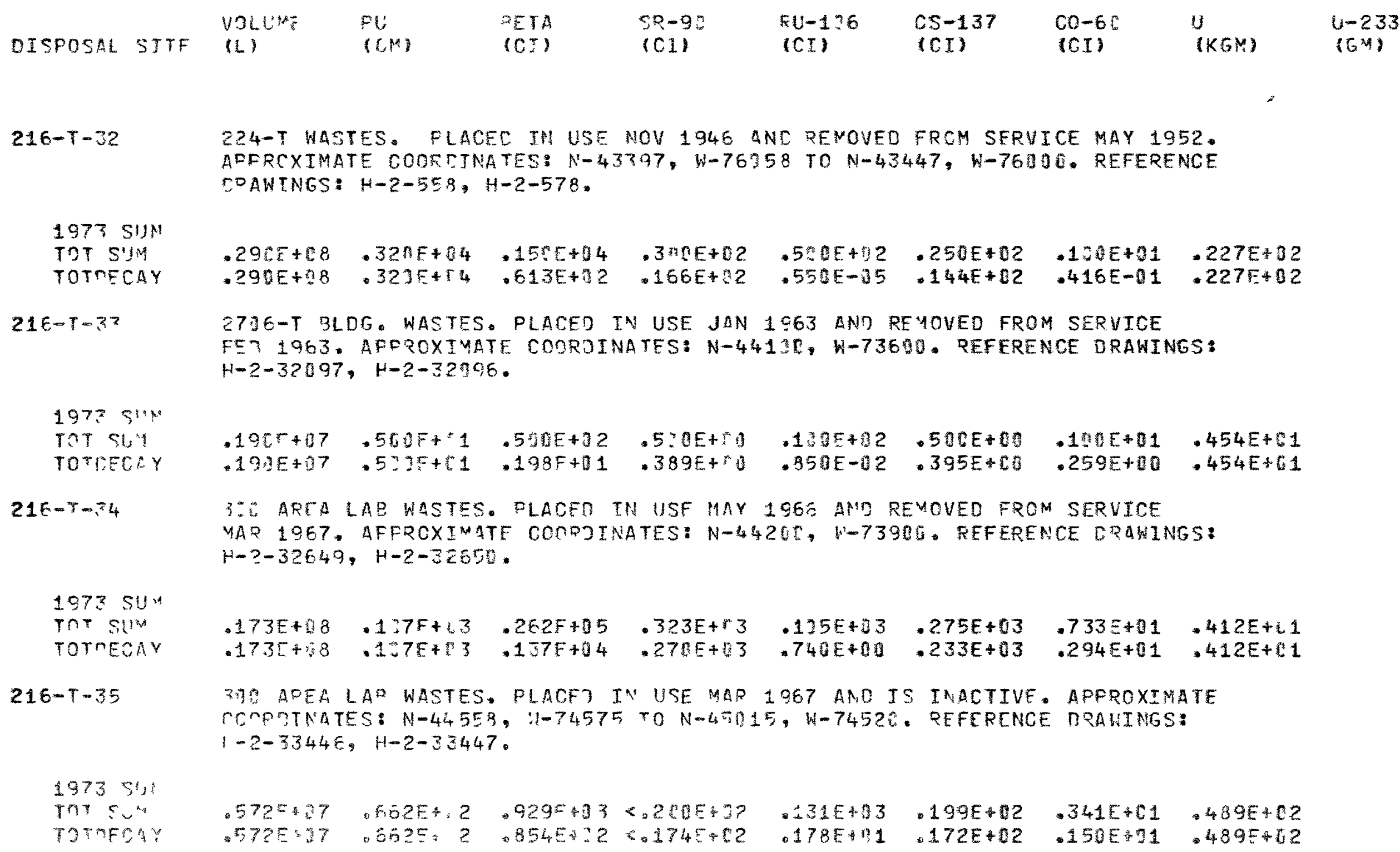


SUMAAPY OF CURRENT, TOTAL, R DECAYFD ACTIVITY DISCHARGED TO CRIBS WITHIN THE CHFMICAL SEPARATIONS AREA CONTROL ZONE THROUGH $9 / 301973$

DISPOSAL SITE

$\begin{array}{lllllllll}\text { VOLUME } & P U & \text { BETA } & S R-90 & \text { PU-1C6 } & C S-137 & C O-60 & U & U-233 \\ (L) & (G M) & \text { (CI) } & (C I) & (C I) & (C I) & (C I) & (K G M) & (G M)\end{array}$

$216-T-36$

1973 SUM TOT SUM TOTDECAY

$215-U-182$

1973 SUM TOT SUM TOTOECAY

$216-U-7$

1973 SUM TOT SUM TOTEECAY

$216-U-4 A$

1973 SUM Tor SUM TOTIECAY

221-T DECON. FACILITY WASTE. PLACED IN USE MAY 1967 AND IS INACTIVE. APDROXIMATE CONFEINATES: N-43093, W-76000 TO N-43093, W-76155. REFERFNCE DRAWINGS: $\mathrm{H}-2-33472$.

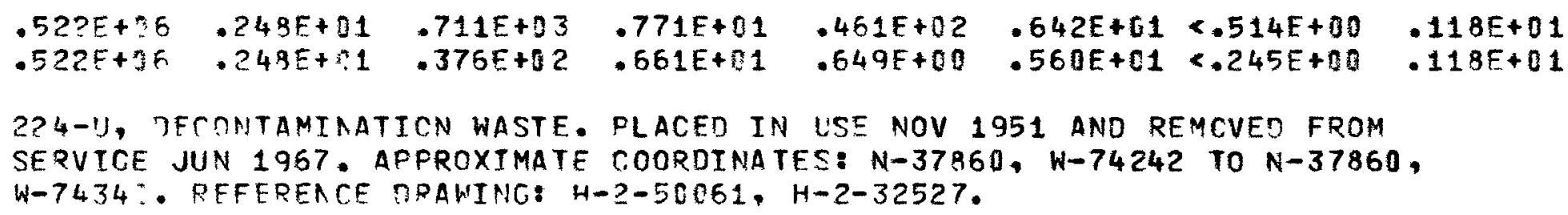

224-U, TECONTAMIAATICN WASTE. PLACED IN USE NOV 1951 AND REMCVEO FROM SERVIGE JUN 1967. APPROXIMATE COORDINATES: N-37860, W-74242 TO N-37860, W-7434:. REFERENCE BPAWING: H-2-50061, H-2-32527.

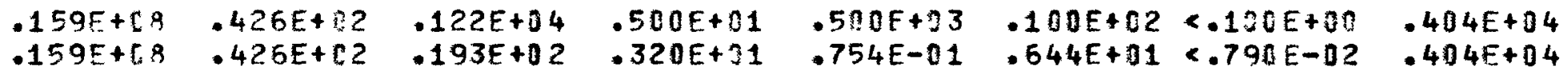

2??-U WASTE. FLACED IN USE JUL 1955 AND EFMOVED FROM SERVICE JUL 1970. APFROXIMATF ROOF[INATES: N-38215, W-7321E. REFFRENCE DRAWINGS: H-2-43081, $S K-2-3735$.

$.545 E+06<.903 E-[2.530 F+01<.330 E-01<.105 E+01.369 E+00$ $.545 F+06<.95 E-r 2 \quad .635 E+00<.242 E-01<.153 E-01 \quad .273 E+00$ 
SUMMAPY DF CUREFNT, TOTAL, \& DECAYED ACTIVITY DISCHARGFD TC CPIBS WITHIN THE CHFMITAL SEPARATIONS AREA CONTROL TONE THROUGH 9/30 1973

$\begin{array}{lllllllll} & \text { VOLUMF } & \text { PU } & \text { PETA } & \text { SR-93 } & \text { PU-1EG } & C S-137 & C O-60 & U \\ \text { DISPOSAL SITE } & \text { (L) } & \text { (GM) } & \text { (CI) } & \text { (CI) } & \text { (CI) } & \text { (CI) } & \text { (CI) } & \text { (KGM) }\end{array}$

$216-U-4 B$

1973 SUM TOT SUM

TOTNECAY

$21 E-U-8$

1973 SUM TOT SUM TOTDECAY

$215-U-12$

1973 SUM TOT SUM

TOTNECAY

$216-u-13$

1973 SUM TOT SUM TOTDECAY
222-U WASTE. PLACED IN USE JAN 1960 AND FEMOVED FROM SERVICE JUL 1970. APFPCXIMATE CODRCINATES: N-39243, W-7310R. RFFERENSE DRAWINGS: SK-2-3337, $\mathrm{H}-2-34762$.

$\begin{array}{lllll}.330 E+05 & .543 E-t 1 & .243 E+31 & .300 E-02 & .334 E+00 \\ .33 n F+05 & .547 E-01 & .577 E+00 & .251 E-02 & .291 E+00\end{array}$

FRDCESS TOND. ANC STACK DRAIN. PLACED IN USE JUN 1952 AND REMOVED FROM SERVICF MAR 1960. APFROXIMATE COORDINATES: N-36860, W-73100. REFERENCE DRAWTNGS: $\mathrm{H}-2-43: 29, \mathrm{H}-2-31321$.

$.379 F+09 \quad .370 E+[3 \quad .270 E+04<.100 E+00 \quad .230 E+03<.100 E+00<.100 E+00 \quad .240 E+05$ $.379 E+09.370 E+[3 \quad .284 E+01<.654 E-11.144 E-02<.672 E-01<.103 E-01 \quad .240 E+05$

UCZ RDOCESS COND. AND STACK DRAIN. PLACEC IN USE APR 1960 AND IS INACTIVE. APDECXIMATF COORTINATES: N-35350, W-7310L. RFFERENCE DRAWINGS: H-2-31321, $\mu-2-32527$.

$.1335+09 \cdot 15 n E+\cdots \quad .853 E+03 \quad .104 E+03 \quad .800 E+02<.100 E+00<-106 E+00 \quad .181 E+34$ $.137 F+09.1: 0 E+1 \quad .175 F+33 \quad .949 E+2.270 E+00<.827 E-01<.337 E-01 \quad .131 E+04$ 241-UR STFAM CLEAIING FIT. PLACER IN USE MAR 1952 AND REMOVED FROM SERVICE MAQ 1955. AFFROXIMATE COORDINATES: N-38200, W-76070. REFERENCE DRAWINGS: $1-2-32527$.

$.1145+35<.13 E+25$

$.114 \tau+05<.1 C O E+10$

$.500 F+02<.130 E+10$

$.130 E+02<.1 C D E+00<.100 E+00$

$.454 E+00$ $.286 F+00<.639 E-81$

$.171 E-04<.657 E-01<.901 E-02$

$.454 \mathrm{~F}+00$ 
SUMMARY OF CURRENT, TOTAL, \& DECAYFD ACTIVITY DISCHARGEO TO CRIBS WITHIN THE CHEMICAL SFFARATIONS AREA CONTROL ZONE THROUGH $9 / 301973$

DISPOSAL SI
216-7-1A
1973-SUM
TOT SUM TOTDECAY

$216-2-3$

1973.SUM TIT SUM TOTDECAY

$216-7-4$

1973 SUM TOT SUM TOTDECAY

$21 E-7-5$

1972 SIJM TIT SUM TITITRAY

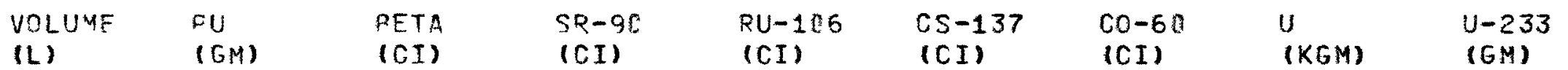

CVERFLOW FROM $21,2,3$. PLACFD IN USE JUN 1949 AND REMOVEO FROM SERVICF MAP 1959. AFPOOXTMATF COOPNINATES: N-39223, $W-765 \% 1$ TO N-39435, h-76815. REFERENCE DRAWINCS: H-2-16459, H-2-32528, H-2-24923.

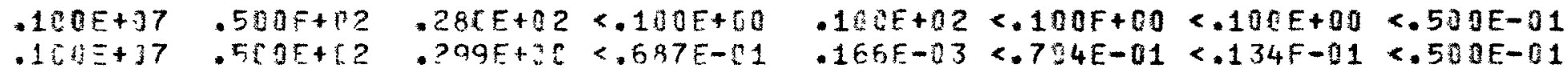
2-6 WASTES. FLACED IN USE JUN 1952 AND REMOVED FROM SERVICE MAR 1959. APPROXIMATE CODPDINATES: N-39435, W-76461. REFERENCE DRAWINGS: H-2-12292, $H-2->4923$.

$.178 \mathrm{~F}+09.570 \mathrm{E}+i 4.105 F+03<.100 \mathrm{~B}+0.400 E+02<.100 E+00<.100 E+00<.500 E-01$ $.178 E+09.570 F+04.325 E+00<.687 E-01.749 E-03<.704 E-01<.134 E-01<.500 E-01$

231-7 LAD WASTF. DLACFO IN USE JUN 1945 ANS RENOVF FROM SERVICE JUN 1945. APORCXIMAT: COOPCINATES: $N-40875$, W-76475. RFFFRENCE ORAWINGS: H-2-511, $H-2-32528$.

$\begin{array}{lllll}<.110 F+05 & .200 E+.1 & .26 E E+01<.100 E+00 & .100 F+01<.100 E+00<.100 E+00<.500 E-01 \\ <.110 E+.5 & .200 F+.1 & .233 E+00<.499 E-61 & .344 E-18<.522 E-01<.2+1 E-02<.500 E-01\end{array}$

231-Z FROCESS WDSTES. FLACEN IN USF JUN 1945 AND REMOVED FROM SERVICE FEB 1947. APPRIX MATF COORTINATES: $N-41920, W-76673$ TO $N-40985, W-76670$.

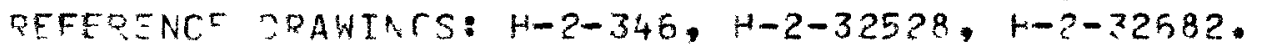
$.310 E+8 \quad-34 C E+3$
$.250 E+33$
$.500 E+01$
$.100 E+23$
- 10DE+02
$.500 E+00<.500 E-01$
$.310 E+" 8 \cdot 340 F+3 \quad \cdot 1 E 3 E+32$
$.253 E+1$
$.641 E-06 \quad .532 E+01$
$.131 E-01<.500 E-01$ 
SUMMAPY OF CUPFENT, TOTAL, DECAYFD ACTIVITY DISCHARGED TC CRIBS WITHIN THE GHEMICAL SFPARATIONS AREA CONTROL TONE THRCUGH $9 / 301973$

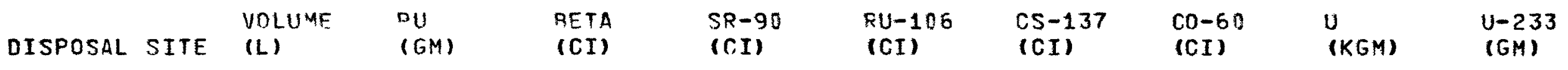

$216-Z-10$

1973 SUM TOT SUM TOTDECAY

$216-7-12$

1973 SUM TOT SUM TOTIECAY

$216-7-16$

1973 SUM TOT SUM TOTAECAY

216-Z - 17

1973 SUM TOT SUM TOTOECAY

231-Z PROCESS AND LABORATORY WASTES. PLACEO IN USE FEB 1945 AND REMOVED FQOM SERVTRE JUN 1945. APPROXIMATE COORDINATES N-40804, $\mathbf{W - 7 6 5 3 5 . ~}$ REFE?FNCF TFAWINES: H-2-32528, H-2-32682.

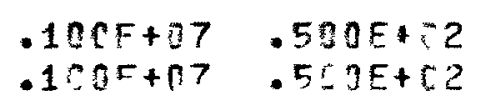

234-5 FPCRESS ANC LAP WASTE. PLACED IN LSE MAR 1959 AND IS STILL ACTIVE. AFCPOXIMATF COORCINATES: N-39400, W-77200 TO N-39480, W-77209. REFERENCE TRAWTNRS: H-2-?0997, H-2-2?995, H-2-70988, H-2-32528.

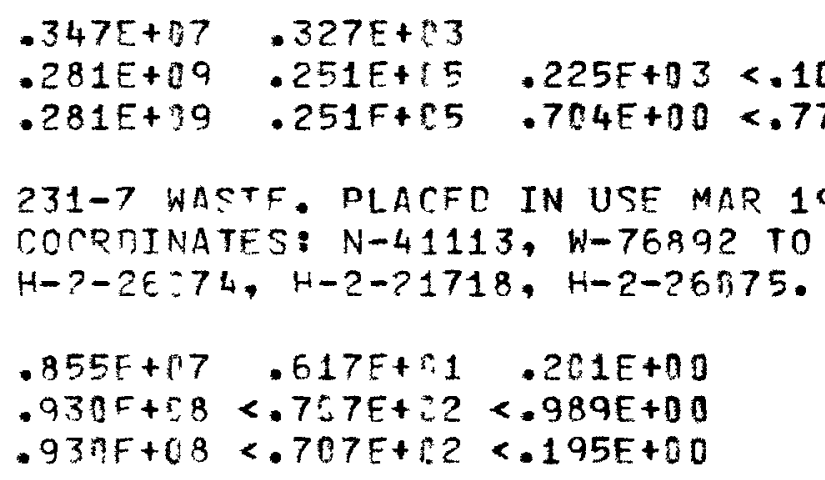

231-7 WASTE. PLACED IN USE FEP 1967 AND REMOVED FROM SERVICE FFB 1968. APPRNXTMATE COORCINATES: $N-40553, W-76372$ TO N-40563, W-76435. RFFFOFNCE DRAWINES: H-2-32632, H-2-32528. 
SUMMARY OF CUFPENT, TOTAL, \& DECAYED ACTIVITY DISCHARGED TO SPECIFIG RETENTION CRIBS WITHIN THE CHEMICAL SEPARATIONS CONTROL TONE THROUGH 9/30 1973

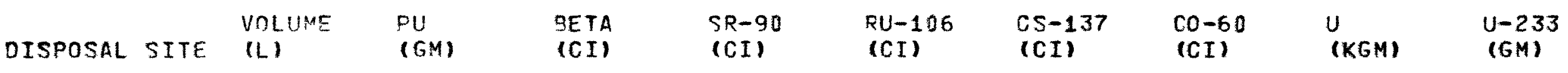

$216-A-1$

1973 SUM TOT SUY

TOTDFCAY

$216-A-2$

1973 SUM TOT SUY

TOTRECAY

$216-A-18$

1973 SUM TOT SUM TOTOECAY

$216-A-19$

1973 SUM TOT SUY TOTOECAY

STAPT-UP HASTES. PLACED IN USE NOV 1955 AND REMOVEO FROM SERVICE DEC 1955. APFREXIMATF COORTINATES: N-41330, W-47150. REFERENCE DRAWINGS: H-2-56016.

$\begin{array}{llllll}.984 E+05<.120 F+00 & .100 E+01<.100 E+00<.100 E+00<.100 E+00<.100 E+00 & .154 E+03 \\ .984 E+75<.100 E+00 & .263 F+00<.639 E-01<.341 E-06<.657 E-01<.901 E-02 & .154 E+03\end{array}$

PUREX ORGANTC. FLACED IN USE MAY 1956 AND REMOVEO FROM SERVICE JAN 1963. APDRCXIMATF CO3RCINATES: N-39515, W-4827\%. REFERFNCE DRAWINGS: H-2-56050, $H-2-56449$.

$.230 E+C 6 \quad .13] E+3 \quad .590 E+03 \quad .200 E+31 \quad \cdot 150 E+03 \quad \cdot 300 E+01.100 E+01 \quad .781 E+02$ $.230 E+06 \quad .138 E+[3 \quad .773 E+31 \quad .14 C E+61 \quad .969 E-02 \quad .215 E+81 \quad .149 E+00 \quad .791 E+02$

START-UP WASTES. PLACED IN USE NOV 1955 AND REMOVEN FROM SERVICE DEC 1955. AFPPDXIMATF CODRCINATES: N-41850, W-47000. REFERENCE DRAWINGS: H-2-55900, $H-2-56119$.

$.438 F+06<.100 F+00 \quad .100 F+02<.100 E+70<.100 E+00<.100 E+00<.100 E+00 \quad .141 E+04$ $.488 E+15<.139 E+C 0.272 F+00<.639 E-01<.341 F-06<.657 E-01<.901 E-02.141 F+04$

START-UP WASTES. FLACED IN USE NOV 1955 AND REMOVEJ FROM SERVICE DEC 1955. APDRCXIMATE COTRCINATES: N-41900, W-46680. REFERENCE DRAWINGS H-2-56521.

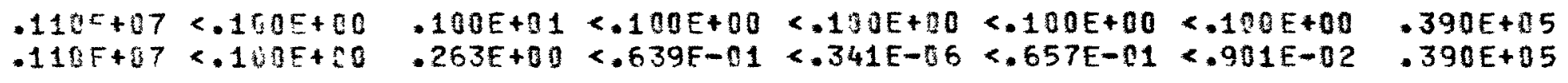


SUMMARY OF CURRENT, TOTAL, \& DECAYED ACTIVITY DISCHARGED TC SFECIFIC RETENTION CRIBS WJTHIN THE CHEMICAL SEFARATIONS CONTROL PONE THROUGH 9/30 1973

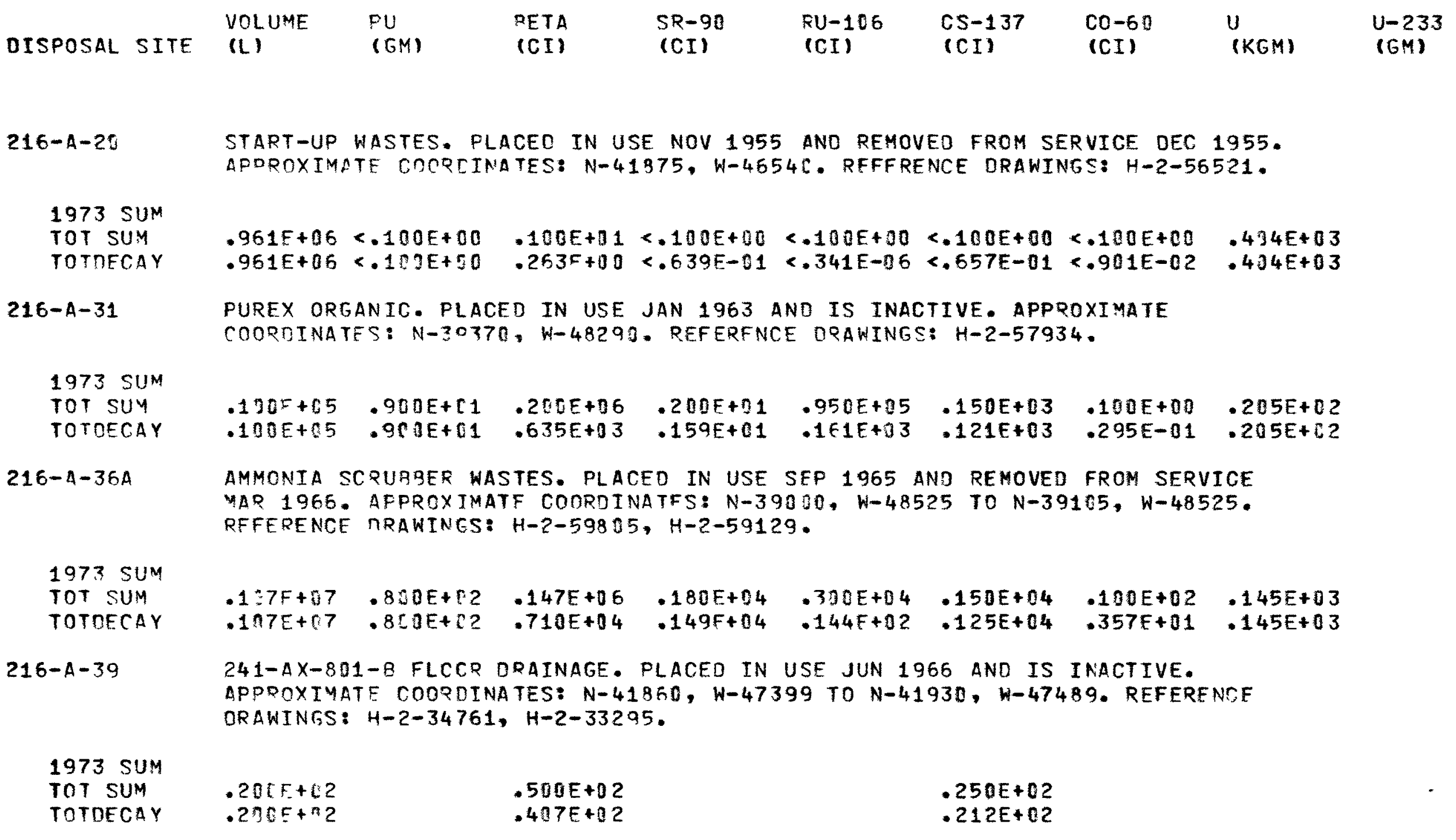


SUMMARY OF CURFENT, TOTAL, DECAYED ACTIVITY DISCHARGED TO SPECIFIC RETENTION CRIBS WITHIN THE CHEMICAL SEPARATIONS CONTROL 7ONE THROUGH $9 / 301973$

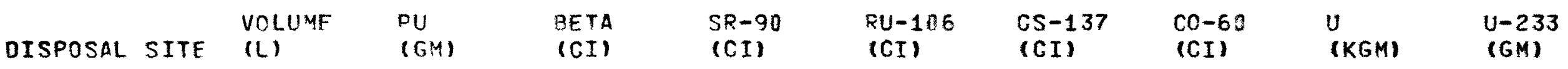

$215-A-4 C$

1973 SUM TOT SUM

TOTEECAY

$216-9-26$

1973 SUM TOT SUM

TOTDECAY

$216-2-21$

1973 SUM TOT SUY

TOTDECAY

$216-P-22$

1973 SUM TOT SUM TOTDECAY
244-AR EMEOTENCY COIL DIVERSION. PLACEO IN USE JAN 1968 AND IS INACTIVE. AFFROXIMATE COORDINATES: N-41519,W-4820G TO N-41858, W-48404. REFERENCE DRAWINCS: $H-2-51979, H-2-63083, H-2-63084$.

$.946 E+16$

$.946 F+.65$

221-U SCAVFNGFE TEP SUFFRNATANT WASTE. PLACER. IN USE ALG 1956 AND REMOVES FROM SFRVICE SEF 1956. APPROXIMATE COORDINATES: $\mathrm{N}-35540, \mathrm{~W}-54190$ TO N-3597, $\mathrm{H}-\mathrm{T} 394 \mathrm{C}$. PFFERFNCE TRAWINGS: $\mathrm{H}-2-3203, \mathrm{H}-2-3204, \mathrm{H}-2-3502 \mathrm{~B}$.

$\begin{array}{llllllll}.468 E+07 & .13 \eta E+01 & .315 E+05 & .790 E+03 & .998 E+04 & .151 E+[4 & .440 E+81 & .352 E+C 3 \\ .468 F+17 & .137 F+01 & .299 E+04 & .517 E+C 3 & .678 E-01 & .101 E+04 & .453 E+00 & .352 E+03\end{array}$

221-U SGAVFNGER TBP SUPERNATANT WASTF. PLACED IN USE SEF 1956 ANM REMOVED FROM SEPVICE DCT 1956. APPROXIMATE COOPCINATES: N-35540, W-54335 TO $\mathrm{A}-3597^{\circ}, \mathrm{H}-5435 \mathrm{5}$. RFFERFNCE DRAWINGS: $\mathrm{H}-2-32^{n} 3, \mathrm{H}-2-3294, \mathrm{H}-2-35020$.

$.467 \equiv+07 \quad .103 E+c 2 \quad .465 E+05 \quad .739 E+03 \quad .147 E+05 \quad .372 E+03 \quad .650 E+01 \quad .675 E+03$ $.467 E+a 7 \quad .103 E+02 \quad .147 E+14 \quad .484 E+03 \quad .999 E-01 \quad .250 E+03 \quad .668 E+00 \quad .675 E+03$

221-U SGAVENGED TPF SUPERNATANT WASTE. PLACED IN USE OCT 1956 AND REMDVED FROM SFRVICF OCT 1956. APPROXIMATE COORDINATES: $N-35540, W-54420$ TO $\mathrm{N}-35979, \mathrm{H}-54171$. REFERENCE DRAWINGS: $\mathrm{H}-2-321, \mathrm{H}-2-3204, \mathrm{H}-2-35020$.

$\begin{array}{llllllll}.474 E+07 & .260 E+01 & .952 E+05 & .438 E+03 & .311 E+05 & .450 E+02 & .134 E+02 & .418 E+03 \\ .474 E+07 & .260 E+01 & .634 E+03 & .267 F+03 & .215 E+00 & .303 E+02 & .133 E+01 & .418 E+03\end{array}$


SUMMARY OF CUPRENT, TOTAL, DECAYED ACTIVITY DISCHARGED TO SFECIFIC RETENTION CRIBS WITHIN THF CHEMICAL SEPARATIONS CONTROL ZONE THROUGH 9/30 1973

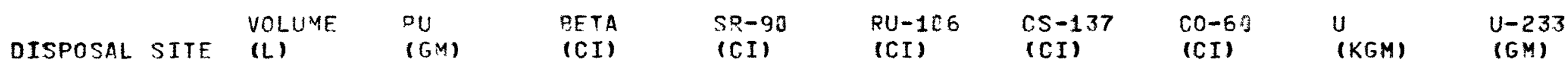

$216-8-23$

197? SUM TOT SUM

TOTDECAY

$215-3-24$

1973 SUM

TOT SUM

TOTDFCAY

$216-9-25$

1973 SUM

TOT SUM

TOTNECAY

$216-R-26$

1973 SUM TOT SUM

TOTDECAY
221-U SCAVENGED TBP SUPERNATANT WASTF. PLACED IN USE OCT 1956 AND REMOVED FROM SFRVICF OCT 1956. APPROXIMATE COORDINATES: N-35300, W-54244 TO $\mathrm{N}-3533: \mathrm{W}-54744$. REFERFNCE DRAWINGS: $4-2-3232, \mathrm{H}-2-35020$.

$\begin{array}{llllllll}.452 F+7 & .180 E+1 & .473 E+05 & .145 E+03 & .150 E+05 & .112 E+03 & .670 E+01 & .156 F+03 \\ .452 E+.77 & .180 F+.1 & .355 E+33 & .949 E+02 & .102 E+30 & .753 E+02 & .699 E+00 & .156 E+03\end{array}$

221-U SCAVFNGEN TBP SUPFRNATANT WASTE. PLACED IN USE OCT 1956 AND REMOVED FROM SERVICE NOV 1956. APPROXIMATE COORDINATES: N-35200, $\mathrm{W}-54244$ TO $N-3525, W-54744$. REFERENCE DRAWINGS: H-2-3232, H-?-3502R.

$\begin{array}{llllllll}.47 E E+07 & .77 J E+01 & .730 E+05 & .181 E+03 & .231 E+35 & .129 E+03 & .103 E+02 & .246 E+03 \\ .470 E+07 & .770 E+01 & .436 E+03 & .118 E+03 & .157 E+00 & .867 E+02 & .106 E+01 & .246 E+03\end{array}$

221-U SCAVFNGED TBF SUPERNATANT WASTE. PLACED IN USE NOV 1956 AND REMOVED

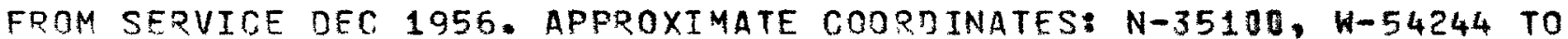
$\mathrm{N}-35135, \mathrm{~W}-54744$. REFERENCE DRAWINGS: H-2-3232, H-2-35028.

$\begin{array}{llllllll}.376 E+57 & .200 E+C 1 & .492 E+35 & .205 E+03 & .156 E+75 & .560 E+02 & .63 E E+01 & .153 E+33 \\ .37 E E+77 & .203 F+01 & .362 E+03 & .134 E+03 & .106 E+30 & .377 E+02 & .710 E+00 & .153 E+03\end{array}$

221-U SCAVENGED TBP SUPERNATANT WASTE. PLACED IN USE DEC 1956 AND REMOVED FRGM SFRVICE FEP 1957. APPOOXIMATE CODRDINATES: N-3500O, W-54244 TO $\mathrm{N}-350 \mathrm{CO}, \mathrm{W}-54744$. REFERENCE DRAWINGS: $\mathrm{H}-2-3232, \mathrm{H}-2-3502 \mathrm{C}$.

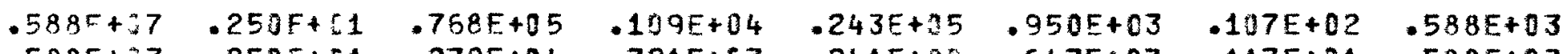
$.588 E+.7 \quad .250 E+01 \quad .272 E+04 \quad .721 E+63 \quad .241 E+070.647 E+03 \quad .117 E+01 \quad .588 E+03$ 
SUMMARY OF CURFENT, TOTAL, IECAYED ACTIVITY DISCHARGED TC SPECIFIC RETENTION CRIBS WITHIN THE CHEMICAL SEPARATIONS CONTROL ZONE THROUGH 9/30 1973

\begin{tabular}{|c|c|c|c|c|c|}
\hline$F$ & $\begin{array}{l}\text { VOLUME } \\
\text { (L) }\end{array}$ & $\begin{array}{l}P U \\
(G M)\end{array}$ & $\begin{array}{l}\text { RETA } \\
\text { (CI) }\end{array}$ & $\begin{array}{l}S R-90 \\
\text { (CI) }\end{array}$ & $\begin{array}{l}2 U-106 \\
(C I)\end{array}$ \\
\hline
\end{tabular}

221-U SCAVENGEN TBP SUFERNATANT WASTE. PLACED IN USE FEB 1957 AND REMOVED FOMM SFRVICF AF? 1957. APPROXIMATE COOPDINATES: $N-349$ r, W-54244 TO $\mathrm{N}-34 \mathrm{ang}, \mathrm{h}-54744$. REFERFNCE DRAWINCS: H-2-3232, H-2-3502W.

1973 SUM TOT SUM TOTOECAY

$216-8-28$

1973 SUM TOT SUM TOTRECAY

$216-3-29$

1973 SUM TOT SUM FOTNFCAY

$216-R-3 ?$

1973 SUM TOT SUM TOTDECAY

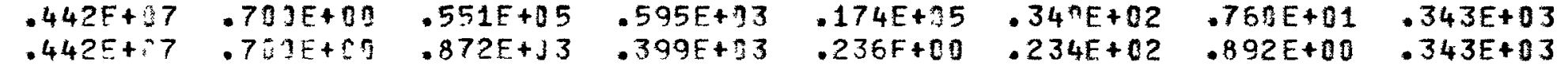

221-U SCAVENGEO TBF SUPERNATANT WASTF. PLACED IN USE APR 1957 AND REMOVED FROM SERVICE JUN 1957. APPROXIMATE CODPDINATES: N-34800, $\mathbf{N}-54244$ TO $\mathrm{N}-34803, \mathrm{~W}-54744$. REFEQENCE DRAWINGS: H-2-3232, H-2-35020.

$\begin{array}{llllllll}.505 E+17 & .563 E+01 & .165 E+05 & .112 E+03 & .522 E+04 & .230 E+02 & .230 E+01 & .300 E+03 \\ .505 E+.7 & .56 J E+01 & .190 E+03 & .751 E+02 & .706 E-01 & .158 E+02 & .270 E+00 & .300 E+03\end{array}$

221-U SCAVENGED TBP SUFERNATANT WASTE. PLACEN IN USE JUN 1957 AND REMOVED FROM SERVICE JUL 1957. APPROXIMATE COORDINATES: N-35972, $N-54900$ TO $\mathrm{N}-35972, \mathrm{~W}-5540 \mathrm{n}$. REFERFNCE BRAWINGS H-z-3336, H-2-3337, H-2-35020.

$\begin{array}{llllllll}.484 E+17 & .110 E+11 & .510 E+05 & .192 E+03 & .161 E+05 & .590 E+02 & .710 E+01 & .344 E+03 \\ .484 E+37 & .110 E+11 & .364 E+03 & .129 E+03 & .219 F+00 & .406 E+02 & .833 E+00 & .344 E+03\end{array}$

221-U SCAVENGEN TPF SUPERNATANT WASTE. PLACED IN USE JUL 1957 AND REMOVED EROM SFPUTCE JUL 1957. AFPROXIMATE COORDINATES: N-35847, W-54900 TO $N-35847, \mathrm{H}-55430$. PEFERENCE DRAWINGS: H-2-3336, H-2-3337, H-2-35020.

$\begin{array}{llllllll}.479 E+137 & .210 E+01 & .160 E+05 & .600 E+03 & .389 E+04 & .337 E+04 & .170 E+01 & .880 E+02 \\ .478 E+07 & .210 E+01 & .526 E+04 & .472 E+03 & .527 E-E 1 & .232 E+04 & .199 E+00 & .890 E+02\end{array}$


SUMMARY OF CURRENT, TOTAL, DECAYFD ACTIVITY DISCHARGED TC SPECIFIC RETENTION CRIBS WITHIN THE CHEMICAL SEFARATIONS CONTROL TONE THROUGH $9 / 301973$

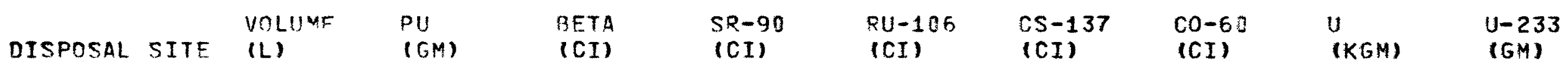

$216-3-31$

1973 SUM TOT SUM

TOTOECAY

$216-3-32$

1973 SUM

TOT SUM

TOTDFCAY

$216-3-33$

1973 SUM TOT SUM

TOTRFCAY

$216-B-34$

1973 SUM TOT SUY

TOTRFCAY
221-U SCAVENGED TBP SUPERNATANT WASTE. PLACED IN USE JUL 1957 ANO RFMOVED FROM SERVICF AUE 1957. AFPROXIMATE COOROINATES: N-35722, W-54903 TO $\mathrm{N}-35722, \mathrm{~W}-55400$. REFERENCE DRAWINGS: $\mathrm{H}-2-3336, \mathrm{H}-2-3337, \mathrm{H}-2-35020$.

$\begin{array}{llllllll}.474 E+37 & .520 E+01 & .192 E+05 & .209 E+03 & .6 n 9 E+174 & .280 E+02 & .270 E+01 & .122 E+03 \\ .474 E+57 & .52 n E+01 & .328 E+03 & .14 B E+.13 & .825 E-01 & .193 E+02 & .317 E+00 & .122 E+03\end{array}$

221-U SCAVENGEC TBF SUFFrNATANT WASTF. PLACEO IN USE AUG 1957 AND REMOVED FROM SERVICF SEF 1957. APPQOXTMATE COOROINATES: $N-35597, W-54900$ TO $\mathrm{N}-35597, \mathrm{~W}-5540 \mathrm{C}$. REFFDENCE DRAWINGS: $\mathrm{H}-2-3336, \mathrm{H}-2-3337, \mathrm{H}-2-35020$.

$\begin{array}{llllllll}.477 E+07 & .26 J E+01 & .120 E+05 & .255 E+03 & .379 E+04 & .126 E+03 & .170 E+01 & .110 E+02 \\ .477 E+.77 & .260 E+01 & .514 E+03 & .171 E+03 & .513 E-01 & .867 E+02 & .199 E+00 & .110 E+02\end{array}$

221-U SCAVFNGFD TBP SUPERNATANT WASTE. PLACED IN USE SEP 1957 AND REMOVED FRJM SERVITF OCT 1957. APPROXIMATE COORIINATES: N-35472, W-54900 TO $\mathrm{N}-35472, \mathrm{~W}-55420$. REFEPENCE DRAWTNGS: H-2-3336, H-2-3337, H-2-35020.

$\begin{array}{llllllll}.474 F+17 & .118 F+: 2 & .102 E+05 & .410 E+02 & .324 E+04 & .273 E+03 & .140 E+01 & .200 E+02 \\ .474 E+77 & .118 E+: 2 & .420 E+03 & .275 E+02 & .439 F-01 & .188 E+03 & .164 E+00 & .200 E+02\end{array}$

2?1-U SCAVFNGF TPF SUPEZNATANT WASTE. PLACED IN USE OCT 1957 AND REMOVED

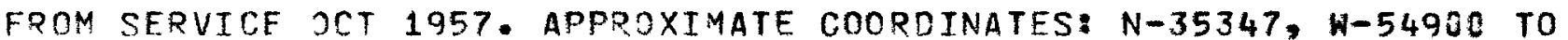
$\mathrm{N}-35347, \mathrm{H}-5540 \mathrm{C}$. PEFERENCE DRAWINGS: H-2-3336, H-2-3337, H-2-35020.

$\begin{array}{llllllll}.497 E+07 & .573 E+01 & .436 E+04 & .410 E+02 & .138 E+04 & .170 E+02 & .600 E+00 & .850 E+02 \\ .4875+07 & .570 E+01 & .798 F+02 & .275 E+02 & .187 E-01 & .117 E+C 2 & .774 E-01 & .850 E+02\end{array}$


SUMMARY OF CURPFNT, TOTAL, \& DECAYED ACTIVITY DISCHARGED TC SPECIFIC RETENTION CRIBS WITHIN THE CHEMICAL SEPARATIONS CONTROL ZONE THROUGH 9/30 1973

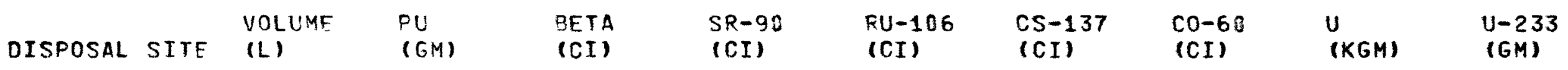

$216-B-35$

1973 SUM TOT SUM TOTDECAY

$216-9-36$

1973 SUM TOT SUM TOTDECAY

$21 E-n-37$

1973 SUM TOT SUM TOTDECAY

$216-9-38$

$1973 \mathrm{SUM}^{\mathrm{M}}$ TOT SUM TOTDECAY
FIRST CYCLE SUPERNATANT. PLACED IN USE FEB 1954 AND REMOVED FROM SERVICE MAP 1954. APP?OXIMATE COOROINATES: N-45273, W-53850 TO N-45273, W-54102. REFFRENCF. DRANINGS: H-2-2431, SK-2-240

$\begin{array}{llllllll}.106 E+07 & .120 F+01 & .179 E+04 & .235 E+03 & .229 E+03 & .427 E+03 & .300 E-01 & .167 E+02 \\ .106 E+07 & .120 E+01 & .819 E+03 & .146 E+03 & .391 E-03 & .274 E+03 & .237 E-02 & .167 E+02\end{array}$

FIRST GYCLE SUPERNATANT. PLACED IN USE MAR 1954 AND REMOVED FROM SERVICE APR 1954. AFPROXIMATE COORDINATES: N-45323, W-53850 TO N-45323, W-54102. PFFFRENCF DFAWINCS; H-2-2431, SK-2-2428.

$.194 E+E 7 \quad .800 E+00 \quad .350 E+04 \quad .485 E+03 \quad .471 E+03 \quad .773 E+03 \quad .700 E-01 \quad .150 E+02$ $.194 E+07 \quad .800 F+00 \quad .156 E+04 \quad .302 E+03 \quad .805 E-03 \quad .496 E+03 \quad .553 E-02 \quad .160 E+02$

FVAFRRATCR BOTTCMS. FLACED IN USE AUR 1954 AND REMOVED FROM SERVICE AUG 1954. AFPRDXIMATE COOROINATES: N-45413, W-53850 TO N-45413, W-54102. REFFRENCF DRAWINES: H-2-2431, SK-2-2408.

$.432 F+07 \quad .200 E+01 \quad .7 n 4 E+174 \quad .160 E+02 \quad .590 E+03 \quad .310 E+C 4 \quad .100 E+01 \quad .363 E+01$ $.432 E+07 \quad .200 E+01 \quad .384 E+74 \quad .997 E+C 1 \quad .855 E-73 \quad .199 E+04 \quad .790 E-01.363 E+01$

FIRST CYCLE SUPFRNATANT. PLACED IN USE JUL 1954 ANO REMOVED FROM SERVICE JUL 1954. APPQOXIMATE COOROINATES: N-45503, W-53850 TO N-45503, N-54102. RFFERENCE DRAWINGS: H-2-2431, SK-2-2408.

$\begin{array}{llllllll}.143 E+07 & .120 E+01 & .582 E+04 & .185 E+04 & .560 E+03 & .508 E+03<.600 E-01 & .424 E+02 \\ .143 F+07 & .120 E+81 & .293 E+04 & .115 E+34 & .957 E-03 & .326 E+03<.474 E-02 & .424 E+02\end{array}$


SUMMARY OF SURRENT, TOTAL, DECAYED ACTIVITY DISCHARGED TC SPECIFIC RETENTION CRIBS WITHIN THE CHEMICAL SEPARATIONS CONTROL ZONE THROUGH 9/30 1973

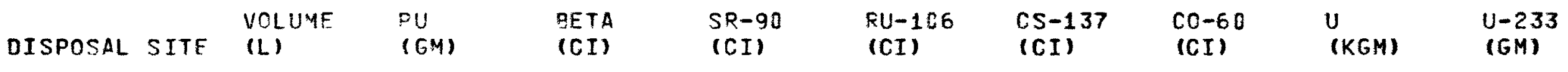

$216-9-39$

1973 SUM

TOT SUM

TOTDFCAY

$216-8-4 C$

1973 SUM

TOT SUM

TOTOECAY

$216-8-41$

1973 SUM TOT SUM

TOTDECAY

$216-8-42$

1973 SUM TOT SUM TOTDECAY
FIRST CYCLE SUPERNATANT. PLACED IN USE DEC 1953 AND REMOVED FROM SERVICE NOV 1954. AFFRCXIMATE COORIINATES: N-45593, W-53850 TO N-45593, W-54102. REFERENCE DRANINGS: H-2-2431, SK-2-2408.

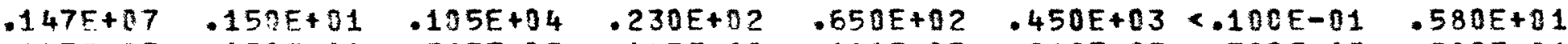

FIRET CYCLF SUPERNATANT. PLACEN IN USE AFR 1954 AND REMOVED FROM SERVICE JUL 1954. APPROXIMATF COOROINATES: N-45683, W-53850 TO N-45683, W-54102. REFERENGF DRAWINGS: $\mathrm{H}-2-2431$, SK-2-24n3.

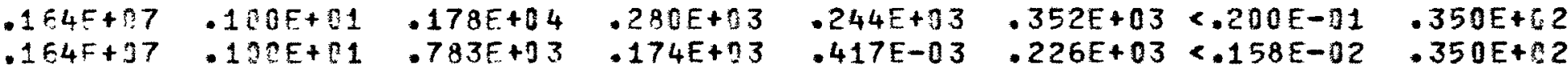

FTRST CYCLE SUPERNATANT. PLACEO IN USE NCV 1954 AND REMOVED FROM SERVICE NOV 1954. APPROXIMATE COORDINATES: N-45773, W-53850 TO N-45773, W-54102. RFFERENCF DRAWINCS: H-2-2431, SK-2-2408.

$\begin{array}{lllllll}.144 F+27 & .300 E+C 0 & .210 E+14 & .470 E+02 & .131 E+03 & .990 E+83<.100 E-91 & .750 E+01 \\ .144 F+07 & .300 E+00 & .116 E+04 & .293 E+C 2 & .224 E-03 & .571 E+03<.790 E-03 & .750 E+01\end{array}$

U FLANT SCAVENGEC WASTE. PLACED IN USE FEP 1955 AND REMOVED FROM SERVICE MAR 1955. APPROXIMATE COOROINATES: N-45273, $N-54152$ TO N-45273, W-54404. PEFERENCE DRAWINGS: $\mathrm{H}-2-2431$, SK-2-2408.

$\begin{array}{llllllll}.150 E+07 & .100 E+02 & .575 E+04 & .110 E+04 & .150 E+04 & .96 E E+02 & .100 E+02 & .680 E+03 \\ .150 E+07 & .100 E+02 & .153 E+04 & .702 E+03 & .511 E-02 & .631 E+02 & .901 E+00 & .680 E+03\end{array}$


SUMMARY OF CURFENT, TOTAL, $\$$ DECAYED ACTIVITY JISCHARGED TO SPECIFIC RETENTION GRIBS WITHIN THE CHEMICAL SEPARATIONS CONTROL TONE THROUGH 9/30 1973

$\begin{array}{llllllllll} & \text { VOLUME } & \text { PU } & \text { EETA } & \text { SR-90 } & R U-106 & C S-137 & C 0-60 & U \\ \text { DISPOSAL SITE } & \text { (L) } & \text { (GM) } & \text { (CI) } & \text { (CI) } & \text { (CI) } & \text { (CI) } & \text { (CI) } & \text { (KGM) } & \text { (GM) }\end{array}$

$216-8-52$

1973 SUM TOT SUM TOTDERAY

$216-9-53 A$

1973 SUM TOT SUM TOTDECAY

$216-B-53 P$

1973 SUM TOT SUM TOTDECAY

$216-8-54$

$1973 \mathrm{SUM}$ TOT SUM TOTOECAY

SCAVENGFO WASTE. PLACED IN USF DEC 1957 AND REMOVED FROM SERVICE JAN 1958. APPOCXIMATF COOREINATES: N-35415, W-5417\% TO N-35415, W-5475\%. REFERENCE DRAWINGS: $4-2-3232, H-2-3336, H-2-352^{2} 2$.

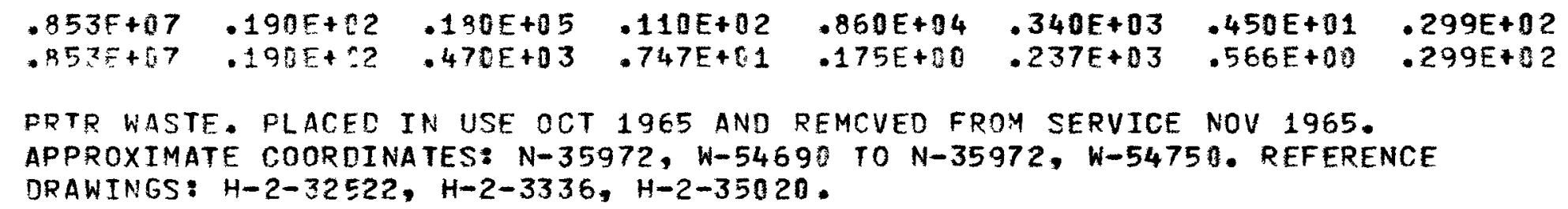

300 AREA WASTE. FLACED IN USE MAR 1963 AND REMOVED FROM SERVICE OCT 1963. APFPOXIMATF COORCINATES: N-35772, W-5455: TO N-35772, W-54750. REFERENCE DRAWINGS: $\mathrm{H}-2-32 \leq 22, \mathrm{H}-2-3336, \mathrm{H}-2-35020$.

$\begin{array}{llllllll}.999 F+06 & .500 E+81 & .240 E+04 & .100 E+00 & .100 E+02 & .100 E+00 & .100 E+00 & .907 E+01 \\ .999 F+16 & .500 E+01 & .237 E+02 & .797 E-01 & .195 E-01 & .808 E-01 & .297 E-01 & .907 E+01\end{array}$


SUMMARY OF CUPRENT, TOTAL, \& DECAYED ACTIVITY DISCHARGED TO SPECIFIC RETENTION CRIBS WITHIN THE CHENICAL SEPARATIONS CONTROL ZONE THROUGH $9 / 301973$

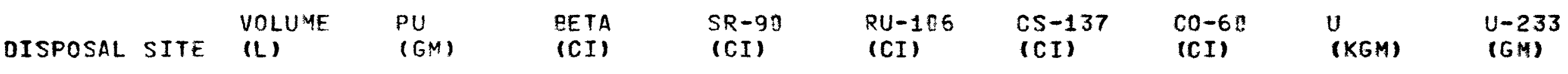

$216-B-56$

$216-8-58$

1973 SUM TOT SUY TOTOECAY

$216-B-59$

1973 SUM TOT SUM TOTOECAY

216-8-6?

1973 SUM TOT SUM

TOTDECAY

$216-C-4$

1973 SUM TOT SUM TOTIFCAY

221-9 ORGANIC GRIB. NOT USED. APPROXIMATE COORDINATES: N-42885, W-52600 TO $\mathrm{N}-42955, \mathrm{~W}-5262 \mathrm{H}$. REFERENCE DRAWINGS: SK-2-19674, H-2-60329.

300 AREA WASTE. FLACED IN USE NOV 1965 AND REMOVED FROM SERVICE JUN 1967. APPROXIMATF COOREINATES: N-35672, W-54550 TO N-35672, W-54750. REFERENCE DRAWINGS: $\mathrm{H}-2-3336, \mathrm{H}-2-33400$.

$\begin{array}{llllllll}.413 E+06 & .673 F+01 & .593 E+12 & .101 E+02 & .720 E+71 & .770 E+01 & .240 E+11 & .912 E+01 \\ .413 E+06 & .670 E+[1 & .307 E+32 & .845 E+01 & .605 E-01 & .653 E+01 & .999 E+00 & .912 E+01\end{array}$

221-8 FMERGENCY COIL DIVERSION. PLACED IN USE MAR 1968 AND IS INACTIVE. APPROXIMATF COORCINATES: N-43001, W-52787 TO N-43284, W-52504. REFERENCE DRAWINGS: $H-2-60310$.
$.477 F+06$
$.500 E+01 \quad \cdot 500 E-01$
$.266 E+00 \quad .439 E-01$
200E-01
$177 E-01$
$.477 E+176$

221-3 CFLL ERAIN CLEANOUT. PLACED IN USE NOV 1967 AND REMOVED FROM SERVICE NOV 1967. APPRCXIMATF COOROINATES: N-42573, W-54179 TO N-42583, W-54178. REFERENCE DRAWINES: $\mathrm{H}-2-60311, \mathrm{H}-2-34303$.

\section{$.189 E+05 \quad .800 E-21 \cdot 155 E+04$}

$800 \mathrm{E}+01$

$.717 E+03$

$.189 E+25 \quad .820 F-1 \quad .447 E+12$

$.693 E+01$

$.717 E+03$

27E-C OPGANIC WASTE. PLACED IN USE JUL 1.55 AND REMOVED FROM SFRVICE MAY 1955. AFPROXIMATE COOROINATES: N-42060, W-50430. REFERENCE DRAWINGS: $H-2-431 i, H-2-4425$.

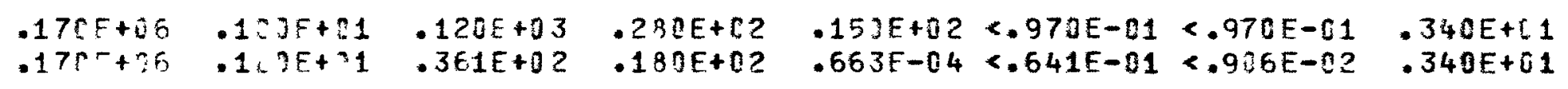


SUMMARY OF CUPRENT, TOTAL, \& DECAYED ACTIVITY DISCHARGED TC SPECIFIC RETENTION CRIBS WITHIN THE GHEMICAL SEPARATIONS CONTROL ZONE THROUGH 9/30 1973

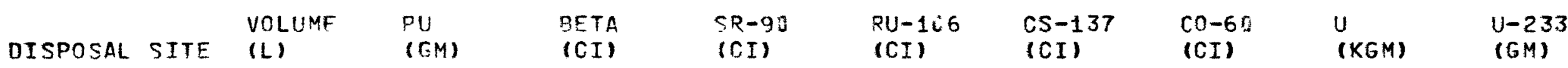

$216-c-6$

1973 SUM TOT SUM

TOTDECAY

$216-5-9$

1973 SUM TOT SUM

TOTDECAY

$216-5-12$

1973 SUM TOT SUM

TOTDECAY

$216-5-13$

1973 SUM

TOT SUM

TOTDECAY
241-CX CONO. PLACED IN USE SEP 1955 AND REMOVED FROM SERVICE SEP 1964. APPRCXIMATF. COORDINATES: N-42015, W-5006E. REFERENCE DRAWINGS: H-2-4425, $H-2-3552 ?$.
$.530 F+06<.100 E+00 \quad .200 E+04.650 E+02 \quad .250 E+02<.100 E+00<.100 E+00 \quad .454 E-01$ $.530 E+06<.100 E+70 \quad .914 E+02.438 E+02 \quad .339 E-02<.638 E-01<.124 E-01 \quad .454 E-01$

START-UF WASTES. FLACEO IN USE NOV 1951 AND REMOVEJ FROM SERVICE FEB 1952. APPROXIMATF COORIINATES: N-35350, W-75300. REFERF.NCE DRAWINGS: $M-26,0$. $5 A 22, \mathrm{H}-2-32525$.

$.102 F+28 \quad .20) E+10.150 E+04 \quad .100 E+1.1 \quad .500 E+02 \quad .120 E+02<.100 E+00.195 E+03$ $.100 E+09 \quad .200 F+01 \quad .159 E+02 \quad .586 E+00 \quad .162 E-04 \quad .727 E+01<.569 E-02 \quad .195 E+03$

291-S STACK WASH. PLACED IN USE JUL 1954 AND REMOVED FROM SERVICE JUL 1954. APPROXIMATF COORDINATES: N-349E5, W-73384. REFERENCE DRAWINGS: $4-2-2436, H-2-32525$.

$.681 E+C 5$. $17 n E+01.550 E+01.100 E+01.190 E+01.100 E+[1<.100 E+00 \quad .499 E+01$ $.591 F+85 \quad .170 E+11 \quad .249 E+01 \quad .623 E+20 \quad .171 E-05 \quad .642 E+00<.790 E-02 \quad .499 E+01$

RFDCX ORGANIC. PLACEC IN USE FEB 1951 ANC IS INACTIVE. APPROXIMATE COOPNINATES: N-34610, W-74620. REFERENCE DRAWINCS: H-2-5385, H-2-5391.

$.500 F+37 \quad .830 E+C 1 \quad .530 E+33 \quad .430 E-01 \quad .100 E+03$

$.500 E+107$

$.800+01$

$127 E+32$

$.310 E-01$

$.293 E+100$

$.500 E+01<.100 E+00<.910 E+02$

$.410 E+11<.316 E-01<.910 E+02$ 
SUMMAPY OF CURFENT, TOTAL, \& DECAYED ACTIVITY DISCHARGED TC SPECIFIC RETENTION CRIBS WITHIN THE CHENICAL SEPARATIONS CONTROL ZONE THROUGH 9/30 1973

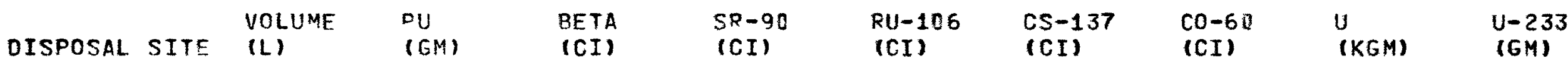

$216-T-5$

1973 SUM TOT SUM

TOTNECAY

$216-T-12$

1973 SUM TOT SUM

TOTDECAY

$216-T-14$

1973 SUM TOT SUM

TOTDECAY

$216-T-15$

1973 SUM

TOT SUM

TOTDECAY
SECOND CYCLE WASTE. PLACFD IN USE MAY 1955 AND REMOVED FROM SERVICE

MAY 1955. AFPOOXTMATE COOROINATES: N-43500, W-76200 TO N-43550, W-76200. RFFEPEACE NRAWINES: H-2-2430, H-2-44510 SHT-3.

$\begin{array}{llllllll}.260 E+07 & .180 E+03 & .208 E+03 & .100 E+01 & .300 E+32 & .700 E+02 & .500 E+01 & .454 E+01 \\ .263 E+07 & .180 F+03 & .900 E+02 & .639 E+00 & .102 E-03 & .460 E+02 & .451 E+00 & .454 E+01\end{array}$

RETFNTIOA BASIN SLUNGE. PLACED IN USE NOV 1954 AND REMCVED FROM SERVICE NOV 1954. APPQOXIMATE CODRDINATES: N-43560, W-75140. REFERENCE DRAWINGS: $\mathrm{H}-2-2430, \mathrm{H}-2-4451 \mathrm{C}$.

$.500 F+07 \quad .13 C E+[1 \quad .550 E+02 \quad .500 E+01 \quad .100 E+02 \quad .100 E+02 \quad .200 E+01 \quad .454 E+02$

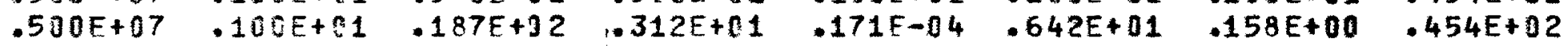

FIRST CYILE SUPERNATANT. PLACED IN USE JAN 1954 ANN REMOVED FROM SERVICE JAN 1954. APPROXIMATE COOPDINATES: N-43780, W-75275 TO N-44030, W-75275. RFFF DFAF TOAUINCS: SK-2-2409,"H-2-35349.

$\begin{array}{llllllll}.130 E+17 & .8875+[0 & .985 E+33 & .600 E+01 & .150 E+02 & .4 E 9 E+03 & .150 E+01 & .303 E+02 \\ .126 E+37 & .993 E+30 & .586 E+03 & .374 E+01 & .256 E-04 & .301 E+03 & .119 E+00 & .303 E+02\end{array}$

FIRST CVCLE SUPFRNATANT. PLACED IN USE FEB 1954 ANO REMOVFO FROM SERVICE FFA 1954. AFPRCXIMATE COORDINATES: N-437ER, W-75185 TO N-440C0, W-75185. REFERENCF DRAWINGS: SK-2-2439, H-2-36849.

$\begin{array}{llllllll}.100 E+47 & .940 E+C 0 & .207 E+04 & .210 E+32 & .120 E+02 & .104 E+04 & .120 E+01 & .272 E+02 \\ .12[E+77 & .947 E+00 & .130 E+04 & . .131 E+32 & .205 E-04 & .665 E+03 & .948 E-01 & .272 E+02\end{array}$


SUMMARY OF CURRENT, TOTAL, DEGAYFD ACTIVITY DISCHARGED TC SPECIFIC RETENTION CRIBS WITHIN THE CHEMICAL SEPARATIONS CONTROL ZONE THRDUGH 9/30 1973

$\begin{array}{lllllllll} & \text { VOLUME } & \text { PU } & \text { RETA } & \text { SR-90 } & R U-166 & \text { CS-137 } & \text { CO-60 } & U \\ \text { DISPOSAL SITE } & \text { (L) } & \text { (GM) } & \text { (CI) } & \text { (CI) } & \text { (CI) } & \text { (CI) } & \text { (CI) } & \text { (KGM) }\end{array}$

$216-T-16$

1973 SUM TOT SUM

TOTDECAY

$216-T-17$

1973 SUM TOT SUM

TOTDECAY

$216-T-18$

1973 SUM TOT SUM TOTDECAY

$216-T-21$

1973 SUM TOT SUM TOTRECAY
FIRST CYCLE SUPERNATANT. PLACED IN USE FEB 1954 ANJ REMOVED FROM SERVICE FE9 1954. APFROXIMATE COORDINATES: N-43760, W-75095 TO N-440CO, W-75095. REFEPENCF DRAWINCS: $\mathrm{SK}-2-2409, \mathrm{H}-2-36849$.

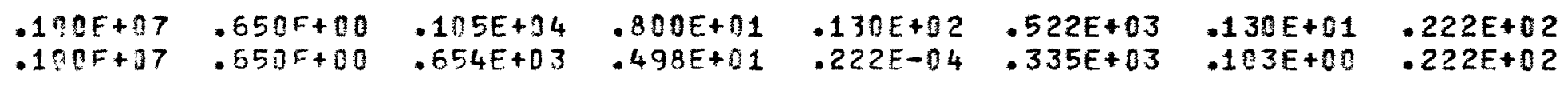

FIRST CYCLE SUPERNATANT. PLACEN IN USE FEB 1954 AND REMOVED FROM SERVICE JUN 1954. APPQOXIMATE COOROINATES: N-43760, W-75045 TO N-44000, W-75045. REFERENCE DPAWINES: SK-2-2439, H-2-36849.

$\begin{array}{llllllll}.735 E+06 & .530 E+00 & .774 E+03 & .300 E+01 & -100 E+02 & .373 E+03 & -100 E+01 & .203 E+02\end{array}$
$.785 E+06 \quad .530 E+00 \quad .464 E+33 \quad .187 E+01 \quad .171 E-34 \quad .239 E+03 \quad .790 E-01 \quad .203 E+02$

SCAVENGED WASTF. DLACED IN USF NOV 1953 AND PFMOVED FROM SERVICE NOV 1953. APPRCXIMATF COORCTNATES: N-42790, W-75300. REFERENCE DRAWINGS: $M-26.0$ SHT-11, H-2-2735, H-2-?733.

$\begin{array}{llllllll}.130 F+17 & .180 E+[4 & .840 E+03 & .700 E+01 & .200 E+03 & .570 E+02 & .100 E+02 & .272 E+02 \\ .100 E+: 7 & .187 E+74 & .791 F+32 & .426 E+11 & .172 E-03 & .358 E+02 & .692 E+00 & .272 E+02\end{array}$

FIRST FYCLE SUPERNATANT. PLACED IN USE JUN 1954 AND REMOVED FROM SERVICE AUG 1954. AFFROXIMATE COORDINATFS: N-41530, W-75450 TO N-41530, W-76690. REFEOFNIE DRAWINES: SK-2-24C9, H-2-36849.

$\begin{array}{llllllll}.450 E+06 & .100 E+C 1 & .821 E+04 & .800 E+01 & .620 E+02 & .400 E+03 & .200 E+01 & .100 E+01 \\ .450 E+15 & .120 E+.11 & .529 E+03 & .498 E+01 & .106 E-03 & .257 E+03 & .158 E+00 & .100 E+01\end{array}$


SUMMARY OF CURFENT, TOTAL, \& DECAYED ACTIVITY DISCHARGED TO SPECIFIC RETENTION CRIBS WITHIN THE CHEMICAL SEPARATIONS CONTROL ZONE THROUGH 9/30 1973

\begin{tabular}{|c|c|c|c|c|c|c|}
\hline OSAL SII & $\begin{array}{l}\text { VOLUME } \\
\text { (L) }\end{array}$ & $\begin{array}{l}F U \\
(G M)\end{array}$ & $\begin{array}{l}\text { BETA } \\
\text { (CI) }\end{array}$ & $\begin{array}{l}S R-90 \\
(C I)\end{array}$ & $\begin{array}{l}\mathrm{RU}-106 \\
(\mathrm{CI})\end{array}$ & $\begin{array}{l}U-233 \\
(G M)\end{array}$ \\
\hline
\end{tabular}

$216-T-22$

1973 SUM TOT SUM

TOTDECAY

$216-T-23$

1973 SUM TOT SUM

TOTNECAY

$216-T-? 4$

1973 SUM TOT SUM

TOTDFCAY

$216-T-25$

1973 SUM TOT SUM

TOTIECAY

FIRST CYCLE SUPERNATANT, PLACED IN USE JUL 1954 AND REMOVED FROM SERVICE AUG 1954. $\triangle F F P C X I M A T E$ CONRDINATES: N-41620, W-76451 TO N-41620, W-75690. REFERENCF TRAWINGS: SK-2-2409, H-2-36849.

$\begin{array}{llllllll}.153 E+07 & .203 E+1 & .394 E+04 & .510 E+02 & .300 E+02 & .185 E+04 & .100 E+01 & .200 E+01 \\ .153 E+37 & .200 E+.1 & .234 E+04 & .318 E+02 & .513 F-04 & .119 E+04 & .790 E-01 & .200 E+01\end{array}$ FIRST CYCLE SUPFENATANT. PLACED IN USE JUL 1954 AND REMOVED FROM SERVICE AUG 1954. APPROXIMATE COORDINATES: N-41710, W-75450 T0 N-41710, W-76690. REFERENCF DRAWINES: $5 K-2-2499, \mathrm{H}-2-36849$.

$\begin{array}{llllllll}.148 E+.7 & .130 E+31 & .350 E+04 & .410 E+02 & .260 E+12 & .133 E+04 & .100 E+01 & .100 E+01 \\ .148 E+77 & .100 E+01 & .169 F+04 & .255 E+02 & .444 E-04 & .854 E+03 & .790 E-01 & .100 E+01\end{array}$

ETPST EYCLE SUPERNATANT. PLACED IN USF ALG 1954 AND REMOVED FROM SERVICE AUG 1954. AFPQOXIMATE COORIINATES: N-41530, W-76453 TO N-41530, W-76690. REFFPENCF ORAWINFS: M-?89? SHT-15, SK-2-2439, H-2-36849.

$.153 F+U 7 \cdot 200 E+N 1 \cdot 435 E+04 \cdot 400 E+02 \quad \cdot 320 E+02.142 E+04.120 E+01.830 E+61$ $.153 E+.7 \quad .200 E+: 1.130 E+04 \quad .249 E+02 \quad .547 E-04 \quad .912 E+C 3 \quad .790 E-01 \quad .830 E+01$

FIRST CYCLE BOTTCMS. PLACET IN USE SEP 1 C54 AND REMOVED FROM SERVICE SEF 1954. AFPRAXIMATE CDORTINATES: N-41350, W-76570. REFERENCE DRAWINGS $M-289 ? S+T-15$

$.300 E+17.100 E+[1.180 E+05$

$30 \mathrm{C}+3$

$130+1$

$.11 \mathrm{E}+05$

$.400 E+B 1$

$100 E+03$

$.890 E+04<.100 E+00$

- $910 E+[0$

$.249 E+1$

$.171 E-03$

$.571 E+04<.790 E-02$

$.910 E+10$ 
SUMMARY OF CURFENT, TOTAL, DECAYED ACTIVITY DISCHARGED TC SFECIFIC RETENTION CRIBS WITHIN THF CHEMICAL SEFARATIONS CONTROL ZONE THROUGH 9/30 1973

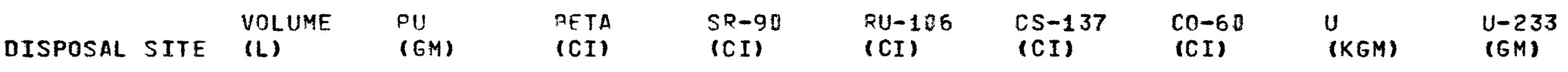

TX-154 DIVERSION OOX. FLACED IN USE JUL 1953 AND REMOVED FROM SERVICE JUL 1953. AFPROXIMATE COORDINATES: N-43880, W-7320\%. REFERENCE DRAWINGS: $H-2-32526$.

1973 SUM TOT SUM TOTNFCAY

$216-U-5$

1973 SUM TOT SUM TOTDECAY

$216-11-6$

1973 SUM TOT SUM TOTIECAY

$216-U-15$

$1973 \mathrm{SuM}$ TOT SUM TOTDECAY

$\begin{array}{llllllll}.190 E+5 & .180 E+01 & .500 F+03 & .400 E+02 & .500 E+02 & .400 E+02 & .100 E+01 & .454 E+01 \\ .190 E+.5 & .123 F+11 & .97 f E+02 & .243 E+02 & .429 E-04 & .251 E+02 & .692 E-01 & .454 E+01\end{array}$

COLD START-UF URANIUM. PLACFD IN USE MAR 1952 AND REMOVED FRCM SERVICE MAR 1952. AFPROXIMATE CODRDINATES: N-39027, W-72922. REFERENCE DRAWINGS: $H-2-32527$.

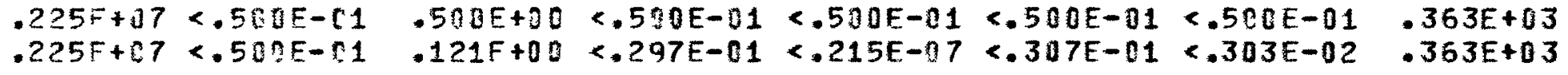

CCLD STAPT-UP URANTUM. PLACFO IN USE MAR 1952 ANO PEMOVEO FRCM SERVICE MAR 1952. AFPROXTMATE COOROINATES: $N-39042, W-73638$ TO N-39079, W-73103. RFFFPEATF TRAWTNES: H-2-32527.

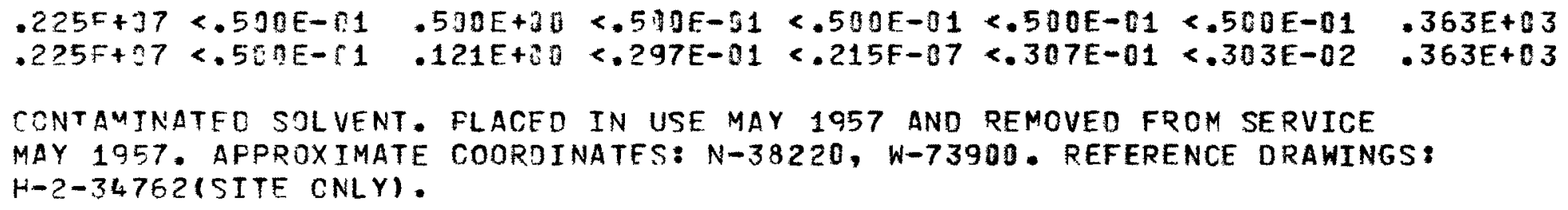


SUMMARY OF CURRENT, TOTAL, \& DECAYED ACTIVITY DISCHARGED TO SPECIFIC RETENTION CRIBS WITHIN THE CHEMICAL SEPARATIONS CONTROL ZONE THROUGH $9 / 301973$

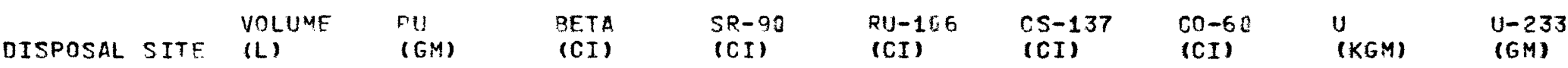

$216-7-1 \& 2$

1973 SUM TOT SUM

TOTDECAY

$216-7-1 A A$

1973 SuM

TOT SUM

TOTOECAY

$216-7-1 A$

1973 SUM TOT SUY

TOTDFCAY

$216-7-1 A C$

1973 SUM

TOT SUM

TOTDECAY
D-6 WASTES. PLACED IN USE JUN 1949 AND REMOVED FROM SERVICE MAY 1969. AFPPOXIMATF CORREINATES: N-39350, W-75550. REFERENCE DRAWINGS: H-2-16459, $H-2-32528$.

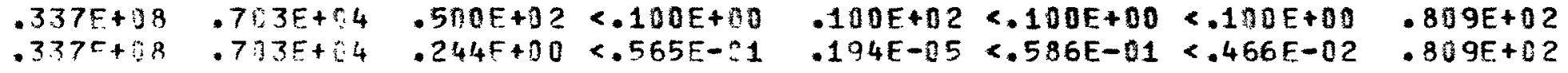

235 AND 242 WASTE. FLACEO IN USE JUN 1964 AND REMOVED FROM SERVICE MAY 1965. AFFROXIMATE CDORDINATES: N-39334, W-76601 TO N-39234, W-76601. REFERENCE DRAWINGS: H-2-16459, H-2-32529, H-2-24923, H-2-27503.

$.190 E+107.300 E+05 \quad .210 E+03<.100 E+00.173 E+03<.100 E+00<.100 E+00<.500 E-01$ $.190 E+.77 .300 E+05 \quad .106 E+01<.797 E-01.337 E+00<.808 E-01<.295 E-01<.500 E-01$

236 AN 242-7 WASTES. PLACED IN USE MAY 1966 AND REMOVED FROM SERVICE CCT 1967. AFFROXIMATE COOROINATES: N-39234, W-76601 TO N-39159, W-76601. REFFPFNCF JRAWINGS: H-2-275U3, H-2-32528,

$.190 F+07 \quad .166 E+05 \quad .105 E+03<.100 E+00 \quad .500 E+02<.100 E+00<.100 E+00<.500 E-01$ $.190 E+7.165 E+75 \quad .111 E+01<.837 E-01 \quad .337 E+00<.846 E-01<.384 E-01<.500 E-01$

236 AND 24?-Z. PLACED IN USE OCT 1967 AND REMOVED FROM SERVICE MAY 1969. APDOOXTYATF COORTINATES: N-39159, W-76601 TO N-39063, W-76601. REFERENCE DRAWINGS: $\mathrm{H}-2-27503, \mathrm{H}-2-3-2528$.
$.141 E+[7 \quad \cdot 108 E+05$
$.141 F+i 7 \quad 108 E+05$ 
TABLE $1-C$

PAGE 48

SUMMARY OF CURFENT, TOTAL, \& DECAYED ACTIVITY DISCHARGED TO SPECIFIC RETENTION CRIBS WITHIN THE CHEMICAL SEPARATIONS CONTROL ZONE THROUGH 9/30 1973

$\begin{array}{lllllllll} & \text { VOLUMF } & \text { PU } & \text { BETA } & \text { SR-90 } & R U-106 & C S-137 & C O-63 & U \\ \text { DISPOSAL SITE } & \text { (L) } & \text { (GM) } & \text { (CI) } & \text { (CI) } & \text { (CI) } & \text { (CI) } & \text { (CI) } & \text { (KGM) }\end{array}$

$216-7-18$

1973 SUM

TOT SUM

TOTIFCAY

236 ANO 242-7. FLACED IN USE APR 1969 ANC IS STILL ACTIVE. APPROXIMATE COORDINATES: $N-38670, W-76830$ TO N-38930, W-75960. REFERENCE DRAWINGS: $\mathrm{H}-2-26293, \mathrm{H}-2-26 \mathrm{n94}, \mathrm{H}-2-36551$.
- $365 E+06$
$.127 E+04$
$.386 F+07 \quad .229 E+05$
$.396 F+7 \cdot 229 E+35$ 
MONTHLY SUMMARY OF LTOUID WASTE IISCHARGED TO GROUNO FROM $1 / 1$ TO $9 / 301973$ AT

DISPOSAL SITE $216-A-B$ WASTE STREAM NESCRIPTIOACRIE

241-A TANK FARM CONDENSATE FROM 11/55 TO 5/58: A-AX FARM CONDENSATE FROM $4 / 66$ TO DATE AND AY FARM CONDENSATE FROM 4/71 TO DATE.

\begin{tabular}{|c|c|c|c|c|c|c|c|c|}
\hline 1973 & $\begin{array}{l}\text { VOLUME } \\
\text { (L) }\end{array}$ & $\begin{array}{l}P U \\
(G M)\end{array}$ & $\begin{array}{l}\text { CETA } \\
\text { (CI) }\end{array}$ & $\begin{array}{l}S R-90 \\
(C I)\end{array}$ & $\begin{array}{l}R U-106 \\
(C I)\end{array}$ & $\begin{array}{l}C S-137 \\
(C I)\end{array}$ & $\begin{array}{l}C O-60 \\
\text { (CI) }\end{array}$ & $\begin{array}{l}\text { U } \\
\text { (KGM) }\end{array}$ \\
\hline $\begin{array}{l}\text { JAN } \\
\text { FEE } \\
\text { MAR } \\
\text { APR } \\
\text { MAY } \\
\text { JUN } \\
\text { JUL } \\
\text { AUE } \\
\text { SEP } \\
\text { OCT } \\
\text { NOV } \\
\text { DEC } \\
\text { ADJ }\end{array}$ & $\begin{array}{l}.332 E+06 \\
.238 E+75 \\
.478 E+16 \\
.194 E+16 \\
.437 E+05 \\
.815 E+06 \\
.958 F+.35 \\
.328 E+06 \\
.535 E+75\end{array}$ & $\begin{array}{r}.812 E-74 \\
.44 E E-34 \\
.190 E-83 \\
.366 E-34 \\
.550 E-05 \\
<.100 E-03 \\
.25 E F-13 \\
<.810 E-\$ 5 \\
<.778 E-85\end{array}$ & $\begin{array}{l}.163 E+00 \\
.144 E+n \\
.827 E+80 \\
.156 E+00 \\
.247 E-01 \\
.993 E+100 \\
.121 F+01 \\
.586 E+00 \\
.216 E+00\end{array}$ & $\begin{array}{r}.124 E-01 \\
.593 E-12 \\
.753 E-01 \\
.128 E-01 \\
.329 E-04 \\
.420 E-01 \\
.192 E-01 \\
.784 E-01 \\
. .157 E-01\end{array}$ & $\begin{array}{r}.509 E-01 \\
.521 E-01 \\
.943 E-01 \\
.220 E-01 \\
.504 E-03 \\
.754 E-01 \\
.111 E+C 0 \\
<.944 E-01 \\
<.252 E-02\end{array}$ & $\begin{array}{l}.114 E-01 \\
.861 E-02 \\
.228 E+00 \\
.421 E-01 \\
.643 E-02 \\
.386 E+00 \\
.478 E+00 \\
.120 E+00 \\
.92 B E-01\end{array}$ & $\begin{array}{l}<.598 E-02 \\
<.735 E-02 \\
<.190 E-03 \\
<.560 E-04 \\
<.128 E-04 \\
<.189 E-03 \\
<.349 E-03 \\
<.568 E-02 \\
<.193 E-03\end{array}$ & $\begin{array}{l}<.349 E-02 \\
<.261 E-02 \\
<.492 E-02 \\
<.200 E-02 \\
<.441 E-03 \\
<.832 E-02 \\
<.104 E-01 \\
<.364 E-02 \\
<.514 E-02\end{array}$ \\
\hline $\begin{array}{l}\text { MONTHLY } \\
\text { MAXIMLIM } \\
\text { CONC }\end{array}$ & & $\begin{array}{l}\text { (UCI/ML) } \\
\text { MAR } \\
.24^{2} E-1.7\end{array}$ & $\begin{array}{l}\text { (UCT/ML) } \\
\text { AUG } \\
.179 E-\$ 2\end{array}$ & $\begin{array}{l}\text { (UCI/ML) } \\
\text { AUG } \\
.239 F-03\end{array}$ & $\begin{array}{l}(U C I / M L) \\
A U G \\
<.288 E-03\end{array}$ & $\begin{array}{l}\text { (UCI/ML) } \\
\text { JUL } \\
.499 E-03\end{array}$ & $\begin{aligned} & (U C I / M L) \\
& F E B \\
< & 309 E-64\end{aligned}$ & $\begin{array}{l}\text { (UCI/ML) } \\
\text { AUG } \\
\text { e.370E-08 }\end{array}$ \\
\hline $\begin{array}{l}\text { AVERAG } \\
\text { CONC }\end{array}$ & & $<.117 E-07$ & $.111 E-92$ & $.673 E-04$ & $<.129 E-03$ & $.352 E-03$ & $<.514 E-05$ & $<.350 E-08$ \\
\hline
\end{tabular}


MONTHLY SUMMARY OF LIQUID WASTE DISCHARGED TO GROUND FROM $1 / 1$ TO $9 / 301973$ AT DISPOSAL SITE 216-A-10 CRIE WASTE STREAM DESCQIPTION- PUREX F-CELL PROCESS CONDENSATE FROM NOVEMBER 1961 TO DATE

\begin{tabular}{|c|c|c|c|c|c|c|c|c|c|}
\hline 1973 & $\begin{array}{l}\text { VOLUME } \\
\text { (L) }\end{array}$ & $\begin{array}{l}P U \\
(G M)\end{array}$ & $\begin{array}{l}\text { BETA } \\
\text { (CI) }\end{array}$ & $\begin{array}{l}S R-90 \\
\text { (CI) }\end{array}$ & $\begin{array}{l}R U-106 \\
\text { (C I ) }\end{array}$ & $\begin{array}{l}\mathrm{CS}-137 \\
\text { (CI) }\end{array}$ & $\begin{array}{l}(0-60 \\
\text { (CI) }\end{array}$ & $\begin{array}{l}U \\
(K G M)\end{array}$ & $\begin{array}{l}U-233 \\
(G M)\end{array}$ \\
\hline $\begin{array}{l}\text { JAN } \\
\text { FEB } \\
\text { MAR } \\
\text { APR } \\
\text { MAY } \\
\text { JUN } \\
\text { JUL } \\
\text { AUG } \\
\text { SEP } \\
\text { OCT } \\
\text { NOV } \\
\text { DEC } \\
\text { AOJ }\end{array}$ & $\begin{array}{l}.151 E+15 \\
.114 E+05 \\
.333 E+75 \\
.114 E+05 \\
.265 E+-5 \\
.757 E+14 \\
.151 E+15 \\
.189 E+05 \\
.189 E+05\end{array}$ & $\begin{array}{l}.656 E-02 \\
.65 E F-C 2 \\
.370 F-02 \\
.655 E-02 \\
.130 E-01 \\
.364 E-02 \\
.94 C E-02 \\
.975 E-02 \\
.975 E-92\end{array}$ & $\begin{array}{l}.712 E-02 \\
.570 E-52 \\
.725 E-82 \\
.312 E-12 \\
.120 E-01 \\
.476 E-02 \\
.440 E-01 \\
.505 E-01 \\
.585 E-01\end{array}$ & $\begin{array}{l}.144 E-03 \\
.177 E-03 \\
.170 E-13 \\
.650 E-04 \\
.147 E-03 \\
.106 E-03 \\
.960 E-03 \\
.850 E-03 \\
.850 E-03\end{array}$ & $\begin{array}{l}.600 E-03 \\
.459 E-03 \\
.440 F-03 \\
.128 E-03 \\
.272 E-03 \\
.169 E-03 \\
.744 E-03 \\
.155 E-02 \\
.155 E-02\end{array}$ & $\begin{array}{l}.225 E-03 \\
.249 E-03 \\
.326 E-03 \\
.113 E-03 \\
.332 E-03 \\
.208 E-03 \\
.203 E-02 \\
.555 E-02 \\
.555 E-02\end{array}$ & $\begin{array}{l}<.180 E-05 \\
<.152 E-05 \\
\times .460 E-05 \\
<.105 E-05 \\
<.633 E-05 \\
<.118 E-05 \\
<.368 E-05 \\
<.360 E-05 \\
<.360 E-05\end{array}$ & $\begin{array}{l}.688 E-03 \\
.573 E-03 \\
.560 E-03 \\
.237 E-03 \\
.623 E-03 \\
.562 E-03 \\
.373 E-02 \\
.448 E-03 \\
.448 E-03\end{array}$ & \\
\hline $\begin{array}{l}73 \text { SUM } \\
\text { PRE SUM } \\
\text { TOT SUM }\end{array}$ & $\begin{array}{l}.155 E+5 \\
.287 E+10 \\
.287 E+1 n\end{array}$ & $\begin{array}{r}.691 E-11 \\
.343 E+03 \\
.343 E+83\end{array}$ & $\begin{array}{l}.185 F+10 \\
.806 F+16 \\
.806 E+06\end{array}$ & $\begin{array}{l}.347 E-02 \\
.147 E+03 \\
.147 E+03\end{array}$ & $\begin{array}{l}.585 E-02 \\
.727 E+E 4 \\
.727 E+04\end{array}$ & $\begin{array}{l}146 E-01 \\
.134 E+03 \\
.134 E+03\end{array}$ & $\begin{array}{r}<.275 E-04 \\
.179 E+103 \\
.179 E+03\end{array}$ & $\begin{array}{l}.787 E-02 \\
.204 E+03 \\
.204 E+03\end{array}$ & $\begin{array}{l}\cdot 277 E+03 \\
.277 E+03\end{array}$ \\
\hline $\begin{array}{l}73 \text { DECAY } \\
\text { PREDECAY } \\
\text { TOTDECAY }\end{array}$ & $\begin{array}{l}1555+16 \\
.287 E+10 \\
.287 E+10\end{array}$ & $\begin{array}{l}.691 E-11 \\
.343 E+03 \\
.343 E+03\end{array}$ & $\begin{array}{l}.152 E+C B \\
.755 E+14 \\
.755 E+04\end{array}$ & $\begin{array}{l}.345 E-52 \\
.125 E+03 \\
.125 F+03\end{array}$ & $\begin{array}{l}.4 c 3 E-02 \\
.145 E+03 \\
.145 E+03\end{array}$ & $\begin{array}{l}.145 E-01 \\
.118 E+03 \\
.118 E+03\end{array}$ & $\begin{array}{r}.263 E-04 \\
.499 E+02 \\
.499 E+02\end{array}$ & $\begin{array}{l}.787 E-02 \\
.204 E+03 \\
.204 E+03\end{array}$ & $\begin{array}{l}.277 E+03 \\
.277 E+03\end{array}$ \\
\hline $\begin{array}{l}\text { MONTHLY } \\
\text { MAXIMUM } \\
\text { CONC }\end{array}$ & & $\begin{array}{l}\text { (UCI/ML) } \\
\text { JUL } \\
-381 E-[4\end{array}$ & $\begin{array}{l}\text { (UCI/ML) } \\
\text { JUL } \\
.291 E-02\end{array}$ & $\begin{array}{l}\text { (UCI/ML) } \\
\text { JUL } \\
.634 E-04\end{array}$ & $\begin{array}{l}\text { (UCI/ML) } \\
\text { AUG } \\
.819 E-C 4\end{array}$ & $\begin{array}{l}\text { (UCI/ML) } \\
\text { AUG } \\
.293 E-03\end{array}$ & $\begin{array}{l}\text { (UCI/ML) } \\
\text { JUL } \\
\times .243 E-06\end{array}$ & $\begin{array}{l}\text { (UCI/ML) } \\
\text { JUL } \\
.820 E-07\end{array}$ & \\
\hline $\begin{array}{l}\text { AVERAGE } \\
\text { CONG }\end{array}$ & & $.273 E-C_{4}$ & $.119 E-02$ & $.224 E-04$ & $.377 E-04$ & $.940 E-104$ & $<.177 E-06$ & $.169 E-07$ & \\
\hline
\end{tabular}


MONTHLY SUMMARY OF LIQUIC WASTE DISCHARGED TO GROUND FROM $1 / 1$ TO $9 / 301973$ AT DISPOSAL SITE 216-A-30 CRIB WASTE STREAM JESCRIPTION- FUPEX COIL AND STEAM CONDENSATE FROM JANUARY 1961 TO DATE.

\begin{tabular}{|c|c|c|c|c|c|c|c|c|c|}
\hline 1973 & $\begin{array}{l}\text { VOLUME } \\
\text { (L) }\end{array}$ & $\begin{array}{l}\text { PU } \\
(G M)\end{array}$ & $\begin{array}{l}\text { BETA } \\
\text { (CI) }\end{array}$ & $\begin{array}{l}S R-90 \\
(C I)\end{array}$ & $\begin{array}{l}\mathrm{PU}-106 \\
(\mathrm{CI})\end{array}$ & $\begin{array}{l}\text { CS }-137 \\
\text { (CI) }\end{array}$ & $\begin{array}{l}\mathrm{CO}-60 \\
(\mathrm{CI})\end{array}$ & $\begin{array}{l}U \\
(K G M)\end{array}$ & $\begin{array}{l}U-233 \\
(G M)\end{array}$ \\
\hline $\begin{array}{l}\text { JAN } \\
\text { FER } \\
\text { MAR } \\
\text { APR } \\
\text { MAY } \\
\text { JUN } \\
\text { JUL } \\
\text { AUG } \\
\text { SEP } \\
\text { OCT } \\
\text { NOV } \\
\text { DEC } \\
\text { ADJ }\end{array}$ & $\begin{array}{l}.109 E+08 \\
.109 E+18 \\
.10 E E+6.8 \\
.994 E+07 \\
.914 E+07 \\
.930 E+37 \\
.819 E+07 \\
.809 E+57 \\
.900 E+07\end{array}$ & $\begin{array}{l}<.10 E F+00 \\
.892 E+ \\
<.1 .9 E+. \\
<.964 E-01 \\
<.903 E-01 \\
<.999 E-11 \\
<.798 E-01 \\
<.784 E-1 \\
<.860 E-11\end{array}$ & $\begin{array}{l}.271 E+00 \\
.135 E+11 \\
.244 F+30 \\
.113 E+00 \\
.154 E+00 \\
.130 E+00 \\
.154 E+100 \\
.132 E+70 \\
.335 E+100\end{array}$ & $\begin{array}{l}.989 E-01 \\
.691 E-01 \\
.592 E-01 \\
.173 E-01 \\
.228 E-01 \\
.228 E-01 \\
.286 E-01 \\
.261 F-01 \\
.107 E+00\end{array}$ & $\begin{array}{r}.266 F-01 \\
.102 E+00 \\
.25 C E-01 \\
.8 E 7 F-02 \\
.232 E-01 \\
.106 E-01 \\
.123 F-01 \\
.137 E-01 \\
.238 E-01\end{array}$ & $\begin{array}{l}.127 E-01 \\
.593 E-01 \\
.195 E-01 \\
.270 E-01 \\
.282 E-01 \\
.319 E-01 \\
.270 E-01 \\
.209 E-01 \\
.166 E-01\end{array}$ & $\begin{array}{l}<.994 E-03 \\
<.121 E-02 \\
<.171 E-02 \\
<.793 E-03 \\
.132 E-02 \\
<.158 E-02 \\
<.147 E-02 \\
<.868 E-03 \\
<.122 E-02\end{array}$ & $\begin{array}{r}<.636 E-01 \\
.124 E+00 \\
.613 E-01 \\
<.604 E-01 \\
.676 E-01 \\
<.516 E-01 \\
<.476 E-01 \\
<.449 E-01 \\
<.261 E+00\end{array}$ & \\
\hline $\begin{array}{l}73 \text { SUM } \\
\text { PRE SUM } \\
\text { TOT SUM }\end{array}$ & $\begin{array}{l}861 F+19 \\
.348 F+10 \\
.356 F+10\end{array}$ & $\begin{array}{l}<.163 F+31 \\
<.716 E+12 \\
<.722 E+12\end{array}$ & $\begin{array}{r}.289 E+01 \\
.544 E+04 \\
.544 E+C 4\end{array}$ & $\begin{array}{l}.435 F+00 \\
.187 E+03 \\
.187 E+03\end{array}$ & $\begin{array}{l}.240 E+00 \\
.196 E+03 \\
.196 E+03\end{array}$ & $\begin{array}{l}.243 E+00 \\
.220 E+03 \\
.220 E+03\end{array}$ & $\begin{array}{r}.112 E-01 \\
.166 E+02 \\
.166 E+02\end{array}$ & $\begin{array}{l}<.783 E+00 \\
<.401 E+02 \\
<.408 E+02\end{array}$ & $\begin{array}{l}<.748 E+01 \\
<.748 E+01\end{array}$ \\
\hline $\begin{array}{l}73 \text { NECAY } \\
\text { PREDECAY } \\
\text { TOTDECAY }\end{array}$ & $\begin{array}{l}951 F+8 \\
.348 F+10 \\
.356 F+10\end{array}$ & $\begin{array}{l}<.163 E+1 \\
<.706 F+2 \\
<.722 F+52\end{array}$ & $\begin{array}{l}.224 E+31 \\
.716 E+33 \\
.713 F+13\end{array}$ & $\begin{array}{l}.430 E+00 \\
.154 E+03 \\
.154 E+03\end{array}$ & $\begin{array}{l}.176 E+00 \\
.936 E+01 \\
.953 E+11\end{array}$ & $\begin{array}{l}.241 E+30 \\
.172 E+03 \\
.172 E+03\end{array}$ & $\begin{array}{r}\times 106 E-31 \\
.437 E+01 \\
.439 E+01\end{array}$ & $\begin{array}{l}<.783 E+00 \\
<.401 E+02 \\
<.408 E+02\end{array}$ & $\begin{array}{l}<.748 E+01 \\
<.748 E+01\end{array}$ \\
\hline $\begin{array}{l}\text { MONTHLY } \\
\text { MAXIMUM } \\
\text { CONC }\end{array}$ & & $\begin{array}{l}\text { (UCT/ML) } \\
F E P \\
.533 E-P 5\end{array}$ & $\begin{array}{l}\text { (UCI/ML) } \\
F E Q \\
.124 E-83\end{array}$ & $\begin{array}{l}(U C I / M L) \\
\text { SFP } \\
.119 E-04\end{array}$ & $\begin{array}{l}\text { (UCI/ML) } \\
F E Q \\
.941 E-35\end{array}$ & $\begin{array}{l}\text { (UCI/ML) } \\
\text { FEB } \\
.544 E-05\end{array}$ & $\begin{array}{l}\text { UUCI/MLI } \\
\text { JUL } \\
\times .179 E-36\end{array}$ & $\begin{array}{l}\text { (UCI/ML) } \\
\text { SEP } \\
<.968 E-04\end{array}$ & \\
\hline $\begin{array}{l}\text { AVERAGF } \\
\text { CONC }\end{array}$ & & $<.11 F F-: 5$ & $.335 E-64$ & $.50 .5 E-05$ & $.279 E-05$ & $.283 E-05$ & $<.130 E-06$ & $<.303 E-09$ & \\
\hline
\end{tabular}




\section{TABLE 5}

MONTHLY SUMMARY DF LIQUID WASTE DISCHARGED TO GROUND FROM 1/1 TO $9 / 301973$ AT DISPOSAL SITE $21 E-B-10 B$ CRIP

WASTE STREAM CESCRIPTIOA- P PLANT R-13 GHANGE HOUSE DECONTAMINATION SINK AND SHOWER WASTE FROM JUNE 1969 TO DATE.

1973

JAN

FEP

MAR

APR

MAY

JUN

JUL

AUG

SEP

OCT

NOV

DEC

ADJ

73 SUM PRF SUM . 212F+65

TOT SUM $.265 F+55$

73 DECAY $.530 F+\$ 4$

PREDECAY $\cdot 212 F+05$

TOTDECAY . 265F+ 55

MONTHLY

MAXIMLIM

CONC

AVERAGE

CONC

$$
\text { VOLUME }
$$

(L)

FU

(GM)

FETA

(CI)

$S R-90$

(CI)

$.456 F-34$

$.262 F-24<.389 E-05$

$.113 E-15$

- $36 \mathrm{RE}-\mathrm{C} 4$

$.272 E-3$

$.757 F+3$

- 11CE-05

$.602 E-66$

$.115 E-04$

$.148 E-76$

$.113 E-54$

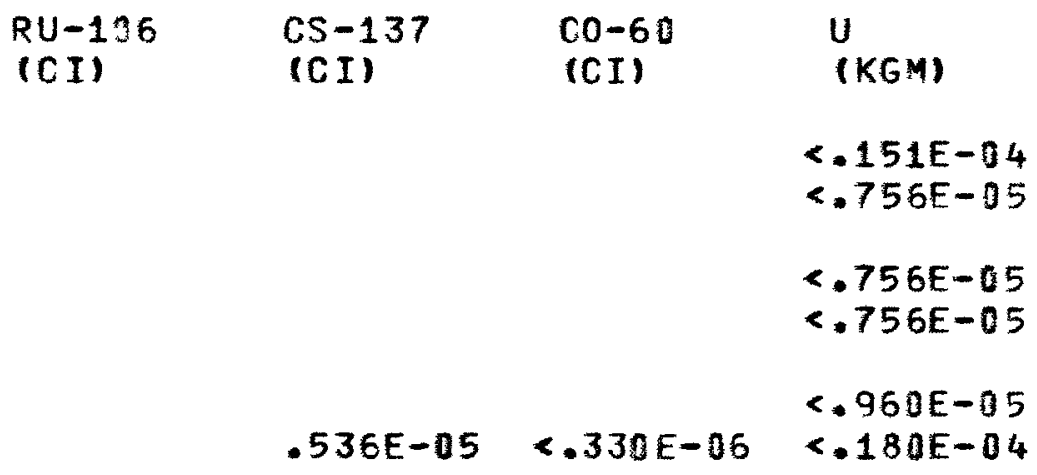

$<.960 E-05$

$<.180 E-04$

$.410 F-15 \quad .403 E-03<.388 E-05$

$<.394 E-34$

$<.435 E-34$

$.133 \mathrm{~F}-72$

$.174 \mathrm{E}-? 2$

$<.327 E-03$

<. 331E-03

$<.169 E-05$

$<.1695-05$

$.536 E-05$

$<.106 E-03$

$<.330 E-06$

$<.450 E-05$

<.780E-06

$.249 E-03$

$.856 E-03$

$111 \mathrm{~F}-0$ ?

$<.382 E-5$

$<.297 F-03$

$<.301 E-03$

<.358E- 06

$.534 E-05$

$<.325 E-05$

$<.334 E-06$

$<.659 E-06$

$<.103 E-63$

(UCI/ML) F.?

$<.513 E-05$

-9P?E-:7

MAY

$.359 E-13$

$.476 E-07 \quad .760 E-04 \quad 0.732 E-06$ $\begin{array}{lll}\text { (UCI/ML) } & (U C I / M L) & \text { (UCI/ML) } \\ \text { AUG } & \text { AUG } & \text { JAN } \\ .354 E-0 E & \times .218 E-06 & \times .566 E-38\end{array}$

$.101 E-05<.623 E-07<.411 E-08$
$<.654 E-94$

$<.665 E-03$

$<.73$ UE -0.3

<. $554 E-04$

$<.665 E-03$

$<.730 \mathrm{E}-63$ 
MONTHLY SUMMARY OF LIOUID WASTF DISCHARGFD TO GROUND FROM $1 / 1$ TO $9 / 301973$ AT DISPOSAL SITE 216-9-12 CRIE

WASTE STREAM DESCRIPTIOA- 221-U BUILDING PROCESS AND STEAM CONDENSATE FROM 1952 THRDUGH 1957. B PLANT CONSTRUCTION WATER FROM MAY 12,1967 TO OCTOBER 1967. 9 PLANT PROCESS CONDFNSATE FROM NOVEMBER 1967 TO DATE.

\begin{tabular}{|c|c|c|c|c|c|c|c|c|}
\hline 1973 & $\begin{array}{l}\text { VOLUME } \\
\text { (L) }\end{array}$ & $\begin{array}{l}D U \\
(G M)\end{array}$ & $\begin{array}{l}\text { EETA } \\
\text { (CI) }\end{array}$ & $\begin{array}{l}S R-90 \\
\text { (CI) }\end{array}$ & $\begin{array}{l}\mathrm{RU}-1 \mathrm{f} 6 \\
\text { (CI) }\end{array}$ & $\begin{array}{l}C S-137 \\
(C I)\end{array}$ & $\begin{array}{l}(0-50 \\
\text { (CI) }\end{array}$ & $\begin{array}{l}U \\
(K G M)\end{array}$ \\
\hline $\begin{array}{l}\text { JAN } \\
\text { FEB } \\
\text { MAR } \\
\text { APR } \\
\text { MAY } \\
\text { JUN } \\
\text { JUL } \\
\text { AUG } \\
\text { SEP } \\
\text { OCT } \\
\text { NOV } \\
\text { DEC } \\
\text { ADJ }\end{array}$ & $\begin{array}{l}.4 C 2 F+07 \\
.316 F+07 \\
.354 E+67 \\
.358 E+87 \\
.352 E+07 \\
.189 F+[7 \\
.584 E+.66 \\
.457 E+.66 \\
.458 E+76\end{array}$ & $\begin{array}{l}.31{ }^{3} E-73 \\
.135 E-12 \\
.262 E-12 \\
.100 E-02 \\
.200 E-02 \\
<.179 F-82 \\
.174 E-02 \\
.39 F F-12 \\
.136 F-37\end{array}$ & $\begin{array}{l}.157 E+r 2 \\
.712 E+01 \\
.957 E+.1 \\
.100 E+02 \\
.418 E+01 \\
.245 E+71 \\
.112 F+.1 \\
.9 n 5 E+70 \\
.712 E+80\end{array}$ & $\begin{array}{l}.295 E+01 \\
.945 E+00 \\
.229 E+01 \\
.181 E+01 \\
.157 E-02 \\
.265 E+00 \\
.130 E+00 \\
.125 F+00 \\
.360 E-01\end{array}$ & $\begin{array}{r}.6 C 5 F+C D \\
.516 E+C 0 \\
.109 E+01 \\
.817 E+00 \\
.158 E+10 \\
.436 F+00 \\
<.168 F+00 \\
<.134 F+00 \\
<.239 E-02\end{array}$ & $\begin{array}{l}.343 E+01 \\
.189 E+01 \\
.128 E+01 \\
.235 E+01 \\
.104 E+01 \\
.529 E+00 \\
.176 E+00 \\
.180 E+00 \\
.254 E+00\end{array}$ & $\begin{array}{r}<.710 F-01 \\
<.118 E+00 \\
.556 E-02 \\
.540 E-02 \\
.505 E-02 \\
.894 E-02 \\
<.180 E+00 \\
<.805 E-02 \\
<.197 E-03\end{array}$ & $\begin{array}{l}<.417 E-01 \\
<.318 E-01 \\
<.357 E-01 \\
<.361 E-01 \\
<.352 E-01 \\
<.191 E-01 \\
<.533 E-02 \\
<.553 E-02 \\
<.459 E-02\end{array}$ \\
\hline $\begin{array}{l}73 \text { SUM } \\
\text { PRE SUM } \\
\text { TOT SUM }\end{array}$ & $\begin{array}{l}.212 F+: 3 \\
.497+79 \\
.51 . F+[9\end{array}$ & $\begin{array}{r}\text { <. } 995 E-02 \\
.374 E+13 \\
.274 F+03\end{array}$ & $\begin{array}{l}.518 F+22 \\
.111 E+55 \\
.112 F+05\end{array}$ & $\begin{array}{r}.955 E+11 \\
<.144 E+73 \\
<.153 F+03\end{array}$ & $\begin{array}{l}\cdot 398 E+11 \\
.367 E+03 \\
.311^{5}+03\end{array}$ & $\begin{array}{l}.111 E+02 \\
.119 E+04 \\
.129 E+04\end{array}$ & $\begin{array}{l}<.321 E+00 \\
<.141 E+01 \\
<.173 F+21\end{array}$ & $\begin{array}{r}<.215 E+00 \\
.209 E+05 \\
.209 E+35\end{array}$ \\
\hline $\begin{array}{l}73 \text { DECAY } \\
\text { PREDFCAY } \\
\text { TOTDECAY }\end{array}$ & $\begin{array}{l}.212 E+09 \\
.497 E+29 \\
.518 F+39\end{array}$ & $\begin{array}{r}<.995 E-02 \\
.374 F+03 \\
.374 E+03\end{array}$ & $\begin{array}{l}.468 E+12 \\
.243 E+04 \\
.248 E+04\end{array}$ & $\begin{array}{r}\quad 844 E+C 1 \\
<-112 E+\$ 3 \\
<.129 F+\$ 3\end{array}$ & $\begin{array}{r}.283 E+01 \\
<.446 E+01 \\
<.729 F+.1\end{array}$ & $\begin{array}{l}.110 E+02 \\
.105 E+04 \\
.106 E+04\end{array}$ & $\begin{array}{l}<.362 E+00 \\
<.862 E+19 \\
<.116 E+01\end{array}$ & $\begin{array}{r}<.215 E+00 \\
.209 E+05 \\
.209 E+05\end{array}$ \\
\hline $\begin{array}{l}\text { MONTHLY } \\
\text { MAXIMUM } \\
\text { CONC }\end{array}$ & & $\begin{array}{l}\text { (UCI/ML) } \\
\text { JUL } \\
.11 \mathrm{mE}-06\end{array}$ & $\begin{array}{l}\text { (UCI/ML) } \\
\text { JAN } \\
.391 F-62\end{array}$ & $\begin{array}{l}\text { (UCI/ML) } \\
\text { JAN } \\
.73^{4} E-03\end{array}$ & $\begin{array}{l}\text { (UCI/ML) } \\
\text { MAR } \\
.36 E-C 3\end{array}$ & $\begin{array}{l}\text { (1)CI/MLI } \\
\text { JAN } \\
.853 E-03\end{array}$ & $\begin{array}{l}\text { (URI/ML) } \\
\text { JUL } \\
\times .172 E-03\end{array}$ & $\begin{array}{l}\text { (UCI/N) } \\
\text { JUL } \\
\text { e.361E-08 }\end{array}$ \\
\hline $\begin{array}{l}\text { AVERAGF } \\
\text { CONC }\end{array}$ & & <.? 8 $9 E-07$ & $.244 E-02$ & $.403 E-03$ & $.198 \mathrm{E}-03$ & $.525 E-03$ & $e .152 E-34$ & $<.337 E-08$ \\
\hline
\end{tabular}


MONTHLY SUMMARY OF LIOUIC WASTE DISCHARGEO TO GROUND FROM $1 / 1$ TO $9 / 301973$ AT DISPOSAL SITE 216-9-50 CRIP WASTE STREAM DESCRIPTIDN- IN-TANK SOLIDIFICATICN NO. 1 CONCENSATE FROM JANUARY 1965 TO DATE.

\begin{tabular}{|c|c|c|c|c|c|c|c|c|}
\hline 1973 & $\begin{array}{l}\text { VOLUME } \\
\text { (L) }\end{array}$ & $\begin{array}{l}P U \\
(G M)\end{array}$ & $\begin{array}{l}\text { EETA } \\
\text { (CI) }\end{array}$ & $\begin{array}{l}S R-90 \\
\text { (CI) }\end{array}$ & $\begin{array}{l}R U-106 \\
(C I)\end{array}$ & $\begin{array}{l}\mathrm{CS}-137 \\
\text { (CI) }\end{array}$ & $\begin{array}{l}\text { CO-60 } \\
\text { (CI) }\end{array}$ & $\begin{array}{l}U \\
\text { (KGM) }\end{array}$ \\
\hline $\begin{array}{l}\text { JAN } \\
\text { FEB } \\
\text { MAR } \\
\text { APR } \\
\text { MAY } \\
\text { JUN } \\
\text { JUL } \\
\text { AUG } \\
\text { SEP } \\
\text { OCT } \\
\text { NOV } \\
\text { DEC } \\
\text { ADJ }\end{array}$ & $\begin{array}{l}.658 E+05 \\
.210 E+66 \\
.227 F+76 \\
.181 E+16 \\
.191 E+.6 \\
.742 F+15\end{array}$ & $\begin{array}{l}<.92 \cap E-05 \\
<.479 E-04 \\
<.312 F-04 \\
<.42 R F-34 \\
<.589 E-14 \\
<.294 E-33\end{array}$ & $\begin{array}{l}.129 E+00 \\
.233 E+C 0 \\
.227 E+20 \\
.231 E+80 \\
.172 E+00 \\
.104 E+00\end{array}$ & $\begin{array}{l}.533 E-04 \\
.152 E-03 \\
.215 E-12 \\
.885 E-04 \\
.115 E-03 \\
.100 E-03\end{array}$ & $\begin{array}{l}.122 E-02 \\
.170 E-02 \\
.185 F-02 \\
.156 F-02 \\
.570 E-03 \\
.308 E-04\end{array}$ & $\begin{array}{l}.655 E-01 \\
.107 E+00 \\
.114 E+00 \\
.115 E+00 \\
.857 E-01 \\
.541 E-01\end{array}$ & $\begin{array}{r}.711 E-05 \\
<.212 E-04 \\
\times .242 E-04 \\
<.285 E-04 \\
\times .293 E-04 \\
<.169 E-04\end{array}$ & $\begin{array}{l}<.388 E-03 \\
<.122 E-02 \\
<.138 E-02 \\
<.115 E-02 \\
<.116 E-02 \\
<.118 E-02\end{array}$ \\
\hline $\begin{array}{l}73 \text { NECAY } \\
\text { PREDECAY } \\
\text { TOTOFCAY }\end{array}$ & $\begin{array}{l}.94 a F+.5 \\
.537 E+.8 \\
.54 E E+C 8\end{array}$ & $\begin{array}{l}<.165 F-62 \\
<.238 F+28 \\
<.230 F+00\end{array}$ & $\begin{array}{l}.104 E+01 \\
.156 E+03 \\
.157 E+03\end{array}$ & $\begin{array}{r}.263 F-02 \\
<.514 E+01 \\
<.514 E+01\end{array}$ & $\begin{array}{l}.473 F-02 \\
.129 E+00 \\
.133 E+C 3\end{array}$ & $\begin{array}{l}.528 E+00 \\
.751 E+02 \\
.757 E+02\end{array}$ & $\begin{array}{l}<.119 E-03 \\
<.142 E+00 \\
<.142 E+00\end{array}$ & $\begin{array}{l}<.647 E-02 \\
<.278 E+00 \\
<.294 E+00\end{array}$ \\
\hline $\begin{array}{l}\text { MONTHLY } \\
\text { MAXIMUM } \\
\text { CONC }\end{array}$ & & $\begin{aligned} & (U C I / M L) \\
J U N & \\
< & 243 E-36\end{aligned}$ & $\begin{array}{l}(U C I / M L) \\
\text { JAN } \\
.193 F-n 2\end{array}$ & $\begin{array}{l}\text { (UGI/ML) } \\
\text { MAR } \\
.951 E-05\end{array}$ & $\begin{array}{l}\text { (UCI/ML) } \\
\text { JAN } \\
.183 E-04\end{array}$ & $\begin{array}{l}\text { (UCI/ML) } \\
\text { JAN } \\
.980 E-03\end{array}$ & $\begin{array}{l}\text { (UCI /ML) } \\
\text { JUN } \\
<.227 E-06\end{array}$ & 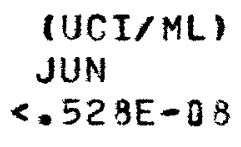 \\
\hline $\begin{array}{l}\text { AVEQAGE } \\
\text { CONC }\end{array}$ & & $<.3 .785-17$ & $.112 \mathrm{E}-[?$ & $.281 E-05$ & $.731 E-05$ & $.563 E-03$ & $<.134 E-06$ & $<.227 E-08$ \\
\hline
\end{tabular}


MONTHLY SUMMARY OF LIOUID WACTF TISCHARGFD TO GROUND FROM 1/1 TO 9/30 1973 AT DISPOSAL SITE 216-R-55 CRIR WASTE STREAM DFSGRIPTION- R DLANT STEAM CONDFNSATE FROM SEFTEMBER, 1967 TO DATE.

\section{3}

VOLUME

$$
\text { (L) }
$$

FU

$$
\text { (GM) }
$$

$.151 E+88$

$.113 E+[8$

- $131 E+08$

- $128 E+R$

- $131 E+08$

$.711 E+67$

- $189 F+07$

- $127 F+17$

$.889 E+106$
BETA

(CI)

$.177 E+21$

$.121 E+11$

$.142 F+01$

$.678 E+60$

$.159 E+1$

$.194 E+71$

$.585 E-51$

$.285 E-1$

$.383 E-02$

\section{$S R-90$}

(CI)

$.134 F+00$

- $215 E+00$

- $19 n+A R$

- $112 E+C D$

. $269 E+00$

- $124 E-01$

$.254 E-01$

. $385 E-02$

. $625 F-03$

\section{RU-106 \\ (CI)}

- $293 E-01$

- $277 E-01$

$.470 F-02$

-159E-01

-171E-01

<.5E2E-.12

- $432 E-02$

$\times .680 E-33$

$<.533 F-03$
CS -137

(CI)

$.732 E+00$

$.364 E+00$

$.387 E+00$

. $195 E+00$

$.996 \mathrm{E}-01$

-395E-0 3

$.399 E-03$

$.423 E-03$
$.455 E+0 G$
$C O-60$
$(C I)$

$<.134 \mathrm{E}-02$

$<.111 E-02$

$<.3 \cap 7 E-02$

$<.207 E-02$

$.207 E-02$

$<.765 E-03$

$<.253 E-03$

$<.119 E-03$

$<.907 E-04$
$U$

(KGY)

C.815E-01

<. 609E-01

$<.715 E-01$

<.881E-01

C.714E-01

<.381E-01

$<.102 E-01$

C. $804 E-02$

<.517E-02

NEC

$A D J$

\section{SUM \\ PRE SUN \\ TOT SUM \\ MONTHLY \\ MAXIMUM}

73 DECAY . 755F+m

PREDECAY . 516E+69

TOTDERAY . 593E+T9

CONC

AVERAGE

CONC

$.766 F+08$

$.516 E+09$

$593 F+r a$
- 17EF-01

$<.414 E+i$

<. $431 F+{ }^{\circ}$

. $17 \mathrm{EF}-\Pi 1$

$<.414 E+10$

$<.431 E+r$

(UCI/ML)

$A P Q$

$.31 F F-.7$

(UCI/ML)

JUN

$.871 E+C$

$.126 E+C 3$

$.135 \mathrm{~F}+03$

$.961 E+00$

$<.527 E+01$

<.524E+D1

$106 E+00$ $<.125 E+1$

$<.136 E+1$

$.778 F+11$

$.470 E+02$

$.548 E+02$

$.949 E+1$

$<.480 E+01$

$<.575 E+01$

$.735 F-01$

C.110E+D0

$<.183 E+\pi 0$

$.272 E-:^{2}$

(UCI/ML)

MAY

(UCI/ML)

FER

$.245 E-\Gamma 5$

$.206 F-84$

0

$.141 E-07$

$.126 F-04 \quad .138 F-05$
$.223 E+01$

- $183 E+02$

$.202 E+02$

$.221 E+D 1$

$.165 E+02$

$.187 E+02$

$<.109 E-01$

$<.314 E+00$

$<.325 F+30$

$<.132 E-01$

$<.167 E+00$

$<.177 \mathrm{E}+0 \mathrm{D}$

(UCI/ML)

JAN

$.433 E-04$

MAR

$<.235 E-36$

$A P R$

$<.229 E-38$

$-435 E+00$

<. $344 E+01$

c. 387E+01

$<.435 E+00$

<. $344 E+01$

$<.387 F+01$

$.292 E-04<.142 E-06<.189 E-08$ 
MONTHLY SUMMAPY OF LIOUID WASTE DISCHARGED TO GROUND FROM $1 / 1$ TO $9 / 301973$ AT DISPOSAL SITE TIF-E-ET CRIE WASTE STRTAM OESC FFTIOA- IN-TANK SOLIDIFICATICN ND. 2 CONDENSATE FROM FEBRUARY 1968 TO DATE.

\begin{tabular}{|c|c|c|c|c|c|c|c|c|}
\hline 1973 & $\begin{array}{l}\text { YOLUMF } \\
\text { (L) }\end{array}$ & $\begin{array}{l}\text { Pu } \\
(6 * 1)\end{array}$ & $\begin{array}{l}\text { TETA } \\
\text { (CI) }\end{array}$ & $\begin{array}{l}S R-90 \\
\text { (CI) }\end{array}$ & $\begin{array}{l}R U-106 \\
(C I)\end{array}$ & $\begin{array}{l}\operatorname{CS}-137 \\
(\mathrm{CI})\end{array}$ & $\begin{array}{l}(0-60 \\
\text { (CI) }\end{array}$ & $\begin{array}{l}U \\
\text { (KGM) }\end{array}$ \\
\hline $\begin{array}{l}\text { JAN } \\
\text { FEP } \\
\text { MAR } \\
\text { APP } \\
\text { MAY } \\
\text { JUN } \\
\text { JUL } \\
\text { AUS } \\
\text { SEP } \\
\text { OCT } \\
\text { NOV } \\
\text { DEC } \\
\text { ADU }\end{array}$ & $\begin{array}{l}.925 E+6 \\
.7 \$ 1 E+D 6 \\
.13 E F+7 \\
.107 E+[77 \\
.765 E+C 6 \\
.312 F+.06\end{array}$ & $\begin{array}{l}<.211 E-03 \\
<.905 E-04 \\
<.129 E-3 \\
<.147 E-3 \\
<.155 E-33 \\
<.577 E-3\end{array}$ & $\begin{array}{l}.201 E+10 \\
.624 E+30 \\
.511 E+70 \\
.219 E+81 \\
.334 E+00 \\
.791 F-21\end{array}$ & $\begin{array}{l}.467 E-03 \\
.522 E-03 \\
.585 E-03 \\
.140 E-03 \\
.147 E-33 \\
.118 E-03\end{array}$ & $\begin{array}{l}.352 E-02 \\
.657 E-02 \\
.402 F-02 \\
.119 E-01 \\
.1 E 5 E-02 \\
.484 E-03\end{array}$ & $\begin{array}{r}.982 E-01 \\
.315 E+00 \\
.295 E+00 \\
.105 E+01 \\
.164 E+00 \\
<.378 E-01\end{array}$ & $\begin{array}{l}<.166 E-03 \\
<.700 E-04 \\
<.168 E-03 \\
<.103 E-0.3 \\
<.627 E-04 \\
<.478 E-04\end{array}$ & $\begin{array}{l}<.533 E-02 \\
<.389 E-02 \\
<.739 E-02 \\
<.651 E-02 \\
<.445 E-02 \\
<.330 E-02\end{array}$ \\
\hline $\begin{array}{l}\text { MONTHLY } \\
\text { MAXI MUM } \\
\text { CONC }\end{array}$ & & $\begin{array}{l}\text { (UCI/ML) } \\
\text { JUN } \\
\text { R. } 114 F-75\end{array}$ & $\begin{array}{l}\text { (UCI/ML) } \\
A P R \\
=2 \cap 3 F-02\end{array}$ & $\begin{array}{l}\text { (UCI/ML) } \\
\text { FER } \\
.715 F-05\end{array}$ & $\begin{array}{l}\text { (URI/ML) } \\
\text { APR } \\
\text {. } 111 E-C 4\end{array}$ & $\begin{array}{l}\text { (UCI/ML) } \\
\text { APR } \\
.983 E-63^{\prime}\end{array}$ & $\begin{array}{l}\text { (UCI/ML) } \\
\text { JAN } \\
\text { \&.19E-BS }\end{array}$ & $\begin{array}{l}\text { (UCI/ML) } \\
\text { JUN } \\
<.352 F-08\end{array}$ \\
\hline $\begin{array}{l}\text { AVERAGE } \\
\text { CONC }\end{array}$ & & $<.158 E-07$ & $.784 E-03$ & $.382 E-176$ & $.549 E-c 5$ & $.382 E-03$ & $<.120 E-06$ & $=.204 E-08$ \\
\hline
\end{tabular}


MONTHLY SUMMARY OF LIQUID WASTE DISCHARGED TO GROUND FROM 1/1 TO 9/30 1973 AT DISPOSAL SITF 216-C-7 CRIP WASTE STREAM NESCRTPTION- CRITICAL MASS LABOPATDRY WASTES FROM 1960 TO DATE.

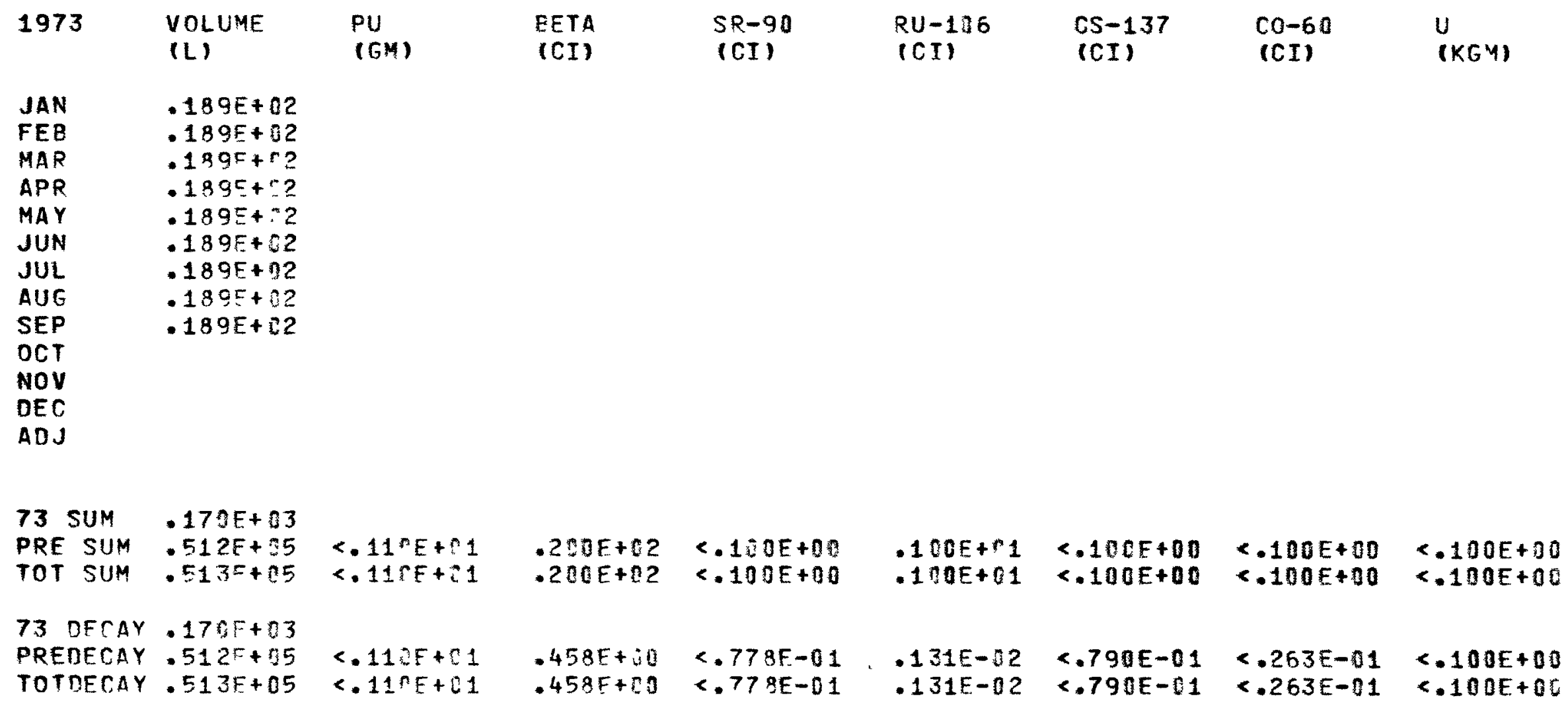

$<.100 E+00$ $<.11 \cap E+01$

(UCI/ML) (UCI/ML)

(UCI/ML)

(UCI/ML)

(UCI/ML)

(UCI/ML)

(UCI/ML) 
MONTHLY SUMMARY OF LIQUTD WASTE DISCHARGED TO GROUND FROM $1 / 1$ TO $9 / 301973$ AT DISPOSAL SITE 216-S-20 CQIE

WASTE STREAM TE SCPIPTIOA- RFDCX LAPORATORY ANO 207-SL RASIN WASTES FPON JANUARY, 1952 TO JANUARY 31,1969 . 222-S LABORATORY WASTES FRCM NCVEMBER 1972 TO OATE.

\begin{tabular}{|c|c|c|c|c|c|c|c|c|}
\hline 1973 & $\begin{array}{l}\text { YOLUME } \\
\text { (L) }\end{array}$ & $\begin{array}{l}\text { PU } \\
(64)\end{array}$ & $\begin{array}{l}\text { RETA } \\
\text { (CI) }\end{array}$ & $\begin{array}{l}S P-90 \\
\text { (CI) }\end{array}$ & $\begin{array}{l}R U-166 \\
\text { (CI) }\end{array}$ & $\begin{array}{l}C S-137 \\
(C I)\end{array}$ & $\begin{array}{l}(0-60 \\
(C I)\end{array}$ & $\begin{array}{l}U \\
(K G M)\end{array}$ \\
\hline $\begin{array}{l}\text { JAN } \\
\text { FEB } \\
\text { MAR } \\
\text { APR } \\
\text { MAY } \\
\text { JUN } \\
\text { JUL } \\
\text { AUG } \\
\text { SEP } \\
\text { OCT } \\
\text { NOV } \\
\text { DEC } \\
\text { ADJ }\end{array}$ & $\begin{array}{l}.717 F+.36 \\
.598 F+.6 \\
.478 F+16 \\
.598 E+16 \\
.598 E+16\end{array}$ & $\begin{array}{r}<126 E-r 3 \\
<.125-r 3 \\
.305 E-13 \\
<.19 E E-3 \\
<.157 E-53\end{array}$ & $\begin{array}{r}.242 E-14 \\
<.373 E-84 \\
<.166 E-04 \\
<.236 E-04 \\
<.336 E-04\end{array}$ & & & & & $\begin{array}{l}<.763 E-02 \\
<.597 F-02 \\
<.510 E-02 \\
<.355 E-02 \\
<.619 E-02\end{array}$ \\
\hline $\begin{array}{l}73 \text { SUM } \\
\text { PRE SUM } \\
\text { TOT SUM }\end{array}$ & $\begin{array}{l}.299 E+C 7 \\
.132 F+C 9 \\
.135 F+C 9\end{array}$ & $\begin{array}{r}<.902 E-13 \\
.171 E+13 \\
.171 F+n 3\end{array}$ & $\begin{array}{r}<.132 F-03 \\
.762 E+24 \\
.762 E+34\end{array}$ & $\begin{array}{l}.451 F+02 \\
.451 E+02\end{array}$ & $\begin{array}{l}-1 D D E+03 \\
.10 C E+13\end{array}$ & $\begin{array}{l}.110 E+03 \\
.110 E+03\end{array}$ & $\begin{array}{l}.141 E+C 1 \\
.141 E+01\end{array}$ & $\begin{array}{r}<.284 E-01 \\
.376 E+02 \\
.376 E+02\end{array}$ \\
\hline $\begin{array}{l}73 \text { DECAY } \\
\text { PREDECAY } \\
\text { TOTDECAY }\end{array}$ & $\begin{array}{l}.299 F+87 \\
.132 F+39 \\
.135 F+139\end{array}$ & $\begin{array}{r}r .902 F-03 \\
.171 E+13 \\
.171 F+03\end{array}$ & $\begin{array}{r}.714 E-04 \\
.256 E+33 \\
.256 E+33\end{array}$ & $\begin{array}{l}.344 E+02 \\
.344 E+02\end{array}$ & $\begin{array}{l}.308 F-31 \\
.388 E-01\end{array}$ & $\begin{array}{l}.835 E+02 \\
.835 E+02\end{array}$ & $\begin{array}{l}.282 E+01 \\
.282 E+0 D\end{array}$ & $\begin{array}{r}\cdot .284 E-01 \\
.376 E+12 \\
.376 E+02\end{array}$ \\
\hline $\begin{array}{l}\text { MONTHLY } \\
\text { MAXIMUM } \\
\text { CONC }\end{array}$ & & $\begin{array}{l}(U C I / M L) \\
M A D \\
.391 E-.7\end{array}$ & $\begin{array}{l}(U C I / M L) \\
F E P \\
<.624 F-07\end{array}$ & (UCI/ML) & (UCI/ML) & $(U C I / M L)$ & $(U C I / M L)$ & $\begin{array}{l}\text { (UCI/ML) } \\
\text { MAR }\end{array}$ \\
\hline $\begin{array}{l}\text { A VERAGE } \\
\text { CONC }\end{array}$ & & $<.185 E-77$ & $<.443 E-57$ & & & & & $<.317 E-08$ \\
\hline
\end{tabular}


MONTHLY SUMMARY TF LIQUID WASTE CISCHARGFI TO GROUND FROM $1 / 1$ TO $9 / 301973$ AT DISFOSAL SITF 216-T-19 CRIF WASTE STREAM NFSERIFTIOA- 242-T FUAPORATOR CONDENSATE FROM DECEMBER $19 E 4$ TO DATE.

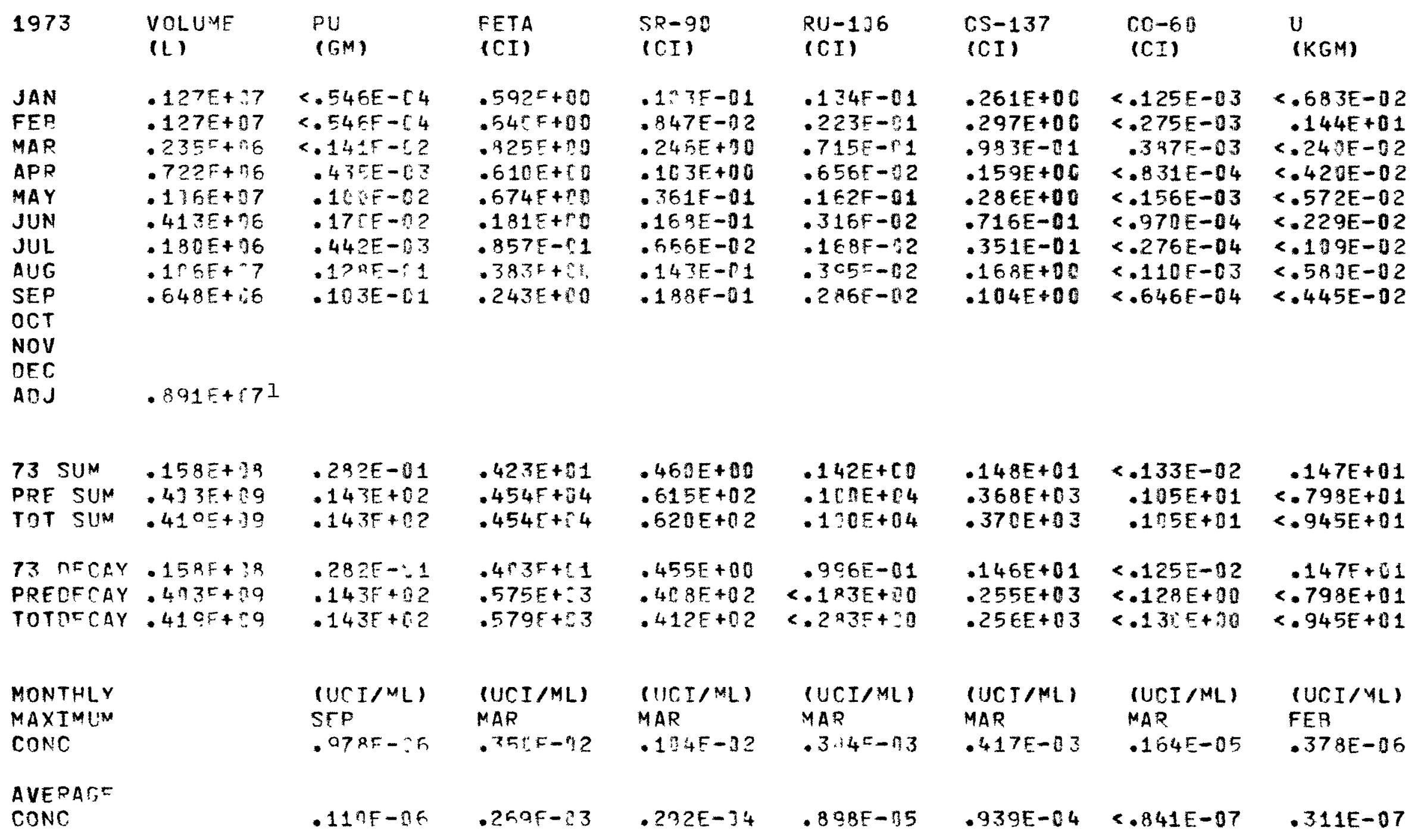

\footnotetext{
$I_{\text {STEAM CONDENSATE. }}$
} 
MONTHLY SUMMARY OF LIQUID WASTF CISCHARGED TO GROUND FROM $1 / 1$ TO $9 / 301973$ AT DISFOSAL SITE 213-Z-12 CRIE WASTE STRFAM JESCRIPTIOA- 234-E, 236, AND LAGORATORY WASTES (0-6) FROM MARCH 1959 TO DATE.

$\begin{array}{lllllllll}1973 & \text { VOLUME } & \text { PU } & \text { BETA } & S R-90 & R U-196 & C S-137 & C 0-60 & U \\ \text { (L) } & \text { (ITM) } & \text { (CI) } & \text { (CI) } & \text { (CI) } & \text { (CI) } & \text { (CI) } & \text { (KGM) }\end{array}$

$\begin{array}{lll}\text { JAN } & .5715+56 & .420 E+52 \\ \text { FEB } & .7525+06 & .520 E+02 \\ \text { MAP } & .122 E+.7 & .520 E+72 \\ \text { APR } & .820 F+56 & .890 F+02 \\ \text { MAY } & .121 E+.6 & .872 E+.22 \\ \text { JUN } & & \\ \text { JUL } & & \\ \text { AUG } & \\ \text { SEP } & \\ \text { OCT } & \\ \text { NOV } & \\ \text { DEC } & & \\ \text { AOJ } & & \end{array}$

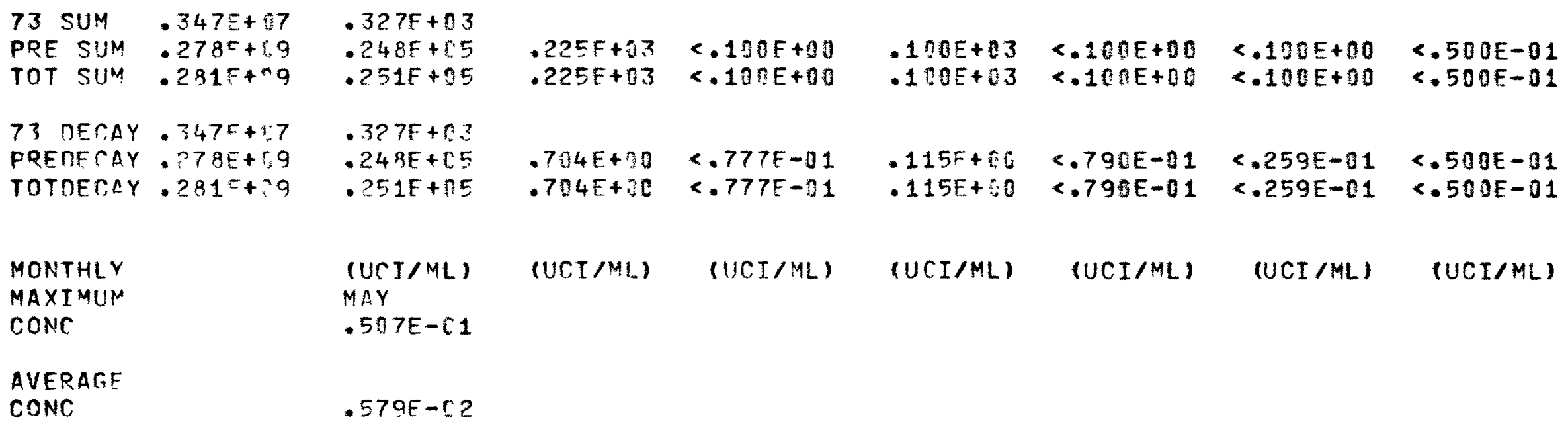


MONTHLY SUMMARY OF LIQUID WASTF DISCHARGED TO GROUND FRON $1 / 1$ TO $9 / 301973$ AT DISPOSAL SITE 21E-7-16 CRIE WASTE STRFAM DESCPTFTION- 231-7 BUILINAG WASTE FROM MARCH 1, 1958 TO DATE.

\begin{tabular}{|c|c|c|c|c|c|c|c|c|}
\hline 1973 & $\begin{array}{l}\text { VOLUME } \\
\text { (L) }\end{array}$ & $\begin{array}{l}P U \\
(G M)\end{array}$ & $\begin{array}{l}\text { BETA } \\
\text { (CI) }\end{array}$ & $\begin{array}{l}S R-90 \\
(C I)\end{array}$ & $\begin{array}{l}R U-106 \\
(C I)\end{array}$ & $\begin{array}{l}C S-137 \\
(G I)\end{array}$ & $\begin{array}{l}(\mathrm{CO}-60 \\
(\mathrm{CI})\end{array}$ & $\begin{array}{l}U \\
\text { (KGM) }\end{array}$ \\
\hline $\begin{array}{l}\text { JAN } \\
\text { FEB } \\
\text { MAR } \\
\text { APR } \\
\text { MAY } \\
\text { JUN } \\
\text { JUL } \\
\text { AUG } \\
\text { SEP } \\
\text { OCT } \\
\text { NOV } \\
\text { DEC } \\
\text { ADJ }\end{array}$ & $\begin{array}{l}.137 E+.7 \\
.150 E+77 \\
.137 E+37 \\
.137 F+77 \\
.134 E+77 \\
.454 E+06 \\
.327 F+56 \\
.3185+.5 \\
.526 E+75\end{array}$ & $\begin{array}{l}.431 E+01 \\
.80 E E+70 \\
.97 \times E+10 \\
.4 D E E-11 \\
.74 E E-1 \\
.161 E-21 \\
.258 E-32 \\
.771 E-2 \\
.798 E-02\end{array}$ & $\begin{array}{l}.139 E+30 \\
.265 E-01 \\
.293 F-1 \\
.184 E-C 2 \\
.361 E-02 \\
.914 E-3 \\
.121 E-83 \\
.352 F-03 \\
.107 E-03\end{array}$ & & & & & \\
\hline $\begin{array}{l}73 \text { SUM } \\
\text { PRE SUM } \\
\text { TOT SUM }\end{array}$ & $\begin{array}{l}.855 E+77 \\
.344 E+29 \\
.930 E+78\end{array}$ & $\begin{array}{r}.617 E+01 \\
<.645 E+02 \\
<.777 E+02\end{array}$ & $\begin{array}{r}.201 E+60 \\
<.787 E+00 \\
<.989 E+00\end{array}$ & & & & & \\
\hline $\begin{array}{l}73 \text { NECAY } \\
\text { PREDECAY } \\
\text { TOTDECAY }\end{array}$ & $\begin{array}{l}.955 F+.7 \\
.844 F+8 \\
.930 E+8\end{array}$ & $\begin{array}{r}.517 E+11 \\
<.645 E+32 \\
<.757 E+22\end{array}$ & $\begin{array}{r}.970 F-1 \\
<.994 E-\pi 1 \\
<.195 E+00\end{array}$ & & & & & \\
\hline $\begin{array}{l}\text { MONTHLY } \\
\text { MAXIMUM } \\
\text { CONC }\end{array}$ & & $\begin{array}{l}\text { (UCI/ML) } \\
\text { JAN } \\
.193 E-03\end{array}$ & $\begin{array}{l}\text { (UCI/ML) } \\
\text { JAN } \\
.101 F-03\end{array}$ & (UCI/ML) & $(U C I / M L)$ & (UCI/ML) & $(U C I / M L)$ & (UCI/ML) \\
\hline $\begin{array}{l}\text { AVERAGE } \\
\text { CONC }\end{array}$ & & $.443 E-04$ & $.235 E-34$ & & & & & \\
\hline
\end{tabular}


MONTHLY SUMMARY OF LTQUTD WASTF DISCHARSED TO GROUND FROM $1 / 1$ TO $9 / 301973$ AT DISPOSAL SITE $216-Z-18$ CRIP (SPECIFIC RETENTION) WASTE STPEAM DESCFIPTION- PLUTCNIUM RFCLAMATION WASTES (236-Z AND 242-Z) FRCM APRIL 4 , 1969 TO DATE.

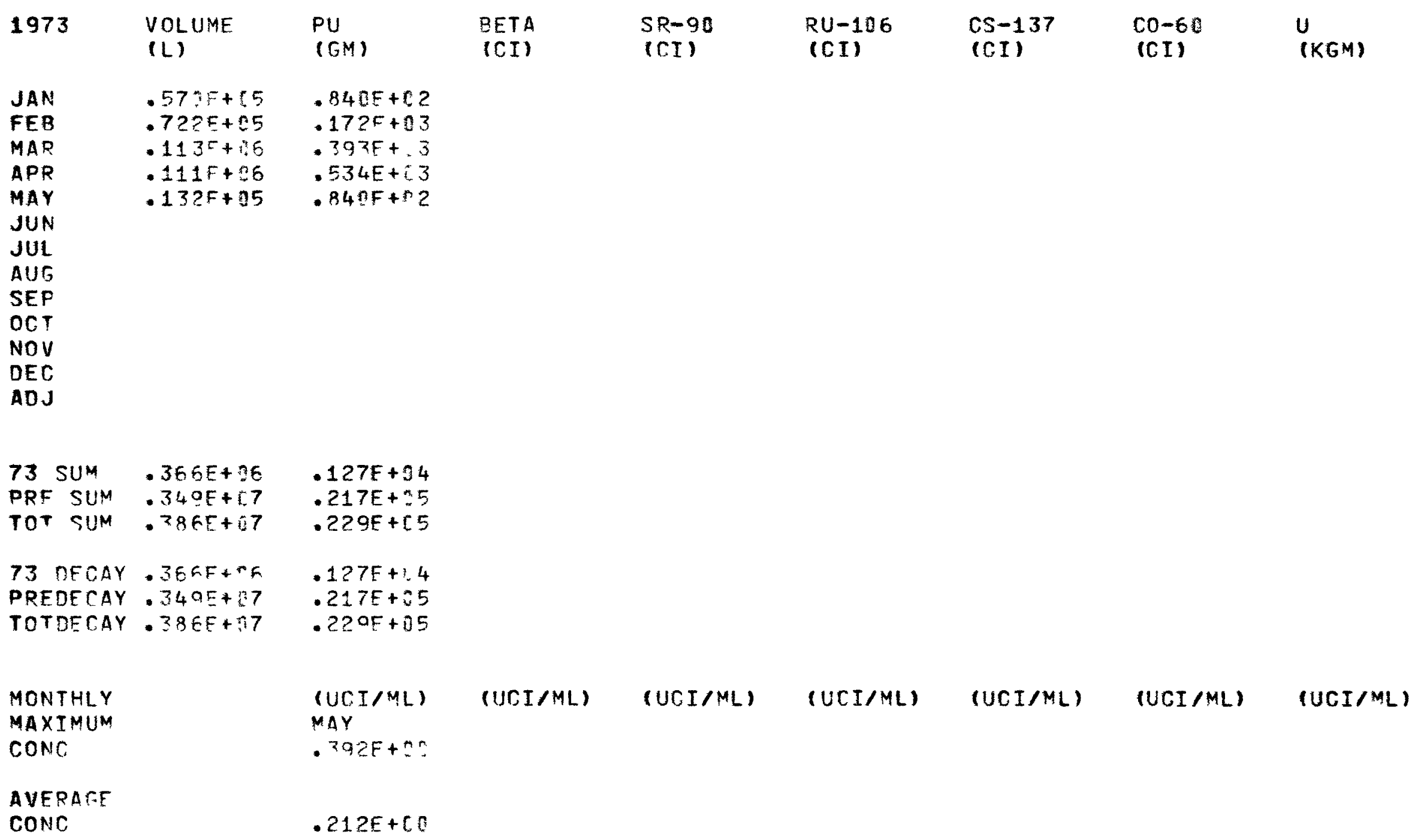

MONTHLY

MAXIMUM

(UCI/ML)

(UCI/ML)

$(U C I / M L)$

$(U C I / M L)$

$(U C I / M L) \quad(U C I / M L)$

(UCI/ML)

$M A Y$

- Ta2F+?

AVERARE

GONC

$.212 E+60$ 


\section{TABLE 16}

MONTHLY SUMMARY OF LJOUTO WASTE DISCHARGED TO GROUND FROM $1 / 1$ TO 9/30 1973 AT

DISFOSAL SITF $216-A-25$ SUMMARY

WASTE STPFAY NESCPTFTTCA- AR VAULT COOLING WATER. AND PUREX PROCESS COOLING WATER.

\begin{tabular}{|c|c|c|c|c|c|c|c|c|c|}
\hline 1973 & $\begin{array}{l}\text { VOLUME } \\
\text { (L) }\end{array}$ & $\begin{array}{l}\text { PU } \\
\left(g^{n}\right)\end{array}$ & $\begin{array}{l}\text { EETA } \\
\text { (CI) }\end{array}$ & $\begin{array}{l}S R-90 \\
(C I)\end{array}$ & $\begin{array}{l}R U-136 \\
(C I)\end{array}$ & $\begin{array}{l}\text { CS }-137 \\
(C I)\end{array}$ & $\begin{array}{l}(0-60 \\
\text { (CI) }\end{array}$ & $\begin{array}{l}U \\
(K G M)\end{array}$ & $\begin{array}{l}U-233 \\
(G M)\end{array}$ \\
\hline $\begin{array}{l}\text { JAN } \\
\text { FEE } \\
\text { MAR } \\
\text { APR } \\
\text { MAY } \\
\text { JUN } \\
\text { JUL } \\
\text { AUG } \\
\text { SEP } \\
\text { OCT } \\
\text { NOV } \\
\text { DEC } \\
\text { ADJ }\end{array}$ & $\begin{array}{l}.701 E+08 \\
.604 E+18 \\
.500 E+88 \\
.472 E+39 \\
.846 E+08 \\
.178=+19 \\
.494 F+68 \\
.341 E+19 \\
.353 E+29\end{array}$ & 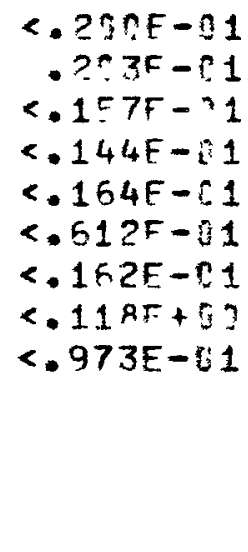 & $\begin{array}{r}<.655 E-32 \\
<.652 F-11 \\
<.742^{r}-32 \\
.791 E-31 \\
.604 E-02 \\
<.669 E-=2 \\
.121 F-[1 \\
<.29 R F-1 \\
.584 E-01\end{array}$ & $\begin{array}{r}<.796 E-04 \\
.148 E-02 \\
<.292 E-04 \\
<.241 E-02 \\
<.127 E-02 \\
<.380 E-02 \\
.957 E-02 \\
.903 E-02\end{array}$ & $\begin{array}{l}<.175 E-03 \\
<.223 F-01 \\
<.626 F-64 \\
<.178 E-01 \\
<.229 E-02\end{array}$ & $\begin{array}{r}<.876 E-04 \\
<.714 E-02 \\
<.310 E-04 \\
.170 E-01 \\
<.389 E-03 \\
<.408 E-02 \\
.184 E-01\end{array}$ & $\begin{array}{l}<.563 E-02 \\
<.485 E-02 \\
<.708 E-02 \\
<.499 E-02 \\
<.181 E-02 \\
<.648 E-03 \\
<.200 E-02 \\
<.375 E-02\end{array}$ & 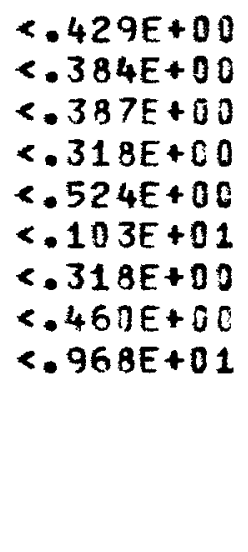 & \\
\hline $\begin{array}{l}73 \text { SUM } \\
\text { PRE SUM } \\
\text { TOT SUM }\end{array}$ & $\begin{array}{l}.197 E+11 \\
.167 F+12 \\
.169 F+12\end{array}$ & $\begin{array}{r}<.380 E+00 \\
.421 E+r 3 \\
.422 E+03\end{array}$ & $\begin{array}{r}<.272 E+3 C \\
.227 F+.16 \\
.227 F+166\end{array}$ & $\begin{array}{r}<.269 E-01 \\
.482 F+03 \\
.482 E+03\end{array}$ & $\begin{array}{l}<.427 E-61 \\
<.740 E+03 \\
<.740 F+03\end{array}$ & $\begin{array}{r}<.471 E-01 \\
.362 E+03 \\
.362 F+03\end{array}$ & $\begin{array}{l}<.308 E-01 \\
<.438 E+02 \\
<.439 E+02\end{array}$ & $\begin{array}{l}e .135 E+02 \\
e .627 E+03 \\
e .641 E+03\end{array}$ & $\begin{array}{l}<.459 E+03 \\
<.459 E+03\end{array}$ \\
\hline $\begin{array}{l}73 \text { DECAY } \\
\text { PREDECAY } \\
\text { TOTDECAY }\end{array}$ & $\begin{array}{l}\cdot 197 F+15 \\
.157 E+12 \\
.1695+12\end{array}$ & $\begin{array}{r}<.335 E+5 \\
.421 E+03 \\
.428 F+03\end{array}$ & $\begin{array}{r}<.24 \pi r+.70 \\
.272 E+24 \\
.272 E+r 4\end{array}$ & $\begin{array}{r}<.369 E-31 \\
.389 E+03 \\
.329 E+03\end{array}$ & $\begin{array}{l}<.296 F-01 \\
<.127 E+n 2 \\
<.127 E+0.2\end{array}$ & $\begin{array}{r}<.468 E-01 \\
.297 E+03 \\
.297 E+03\end{array}$ & $\begin{array}{l}<.299 E-01 \\
<.217 E+02 \\
<.217 E+02\end{array}$ & $\begin{array}{l}<.135 E+02 \\
<.627 E+0.3 \\
<.641 E+03\end{array}$ & $\begin{array}{l}<.459 E+03 \\
<.459 E+03\end{array}$ \\
\hline $\begin{array}{l}\text { MONTHLY } \\
\text { MAXIMUM } \\
\text { CONC }\end{array}$ & & $\begin{array}{l}(U S I / M L) \\
\text { AUr } \\
<.213 E-07\end{array}$ & $\begin{array}{l}(U C I / M L) \\
\triangle P R \\
.1 F R E-15\end{array}$ & $\begin{array}{l}(U C T / M L) \\
J U L \\
<.520 E-07\end{array}$ & $\begin{array}{l}\text { (UCI/ML) } \\
\text { APR } \\
\text { <.377F-106 }\end{array}$ & $\begin{array}{l}\text { (UCI/ML) } \\
\text { APR } \\
.359 E-R E\end{array}$ & $\begin{array}{l}\text { (UCI /ML) } \\
\text { MAR } \\
<.116 E-06\end{array}$ & $\begin{array}{l}\text { (UCI/ML) } \\
\text { SEP } \\
<.913 F-08\end{array}$ & \\
\hline $\begin{array}{l}\text { AVERAGE } \\
\text { COAC }\end{array}$ & & $<.118 F-[7$ & $<.1385-06$ & $<.136 E-07$ & $<.216 E-07$ & $<.239 E-07$ & $<.156 E-07$ & $<.228 E-08$ & \\
\hline
\end{tabular}

$I_{\text {VOLUME ADJ. INCLUDES }} .729+09$ FROM 200 EAST POWERHOUSE. 
MONTHLY SUMMARY OF LIQUTD WASTF OISCHARGFD TO GROUND FROM $1 / 1$ TO $9 / 301973$ AT DISPOSAL STTE $216-4-25$ FONC WASTE STREAM DFSRPIPTION- FURFX PQCCFSG COOLING WATER FROM DFCEMBER 1957 TC DATE.
1973

VOLUME

(L)

PU

(GM)

\section{$.625 E+19$}

$.529 E+18$

$.5325+3$

- $396 E+B R$

$.770 E+78$

$.168 E+09$

$.499 E+08$

$.315 E+79$

- $323 E+09$
$<.20 C F-191$

- 203E- 1

$<.15 F E-01$

$<.143 E-01$

$<.159 E-61$

<. 50 वE- 1

$<.162 E-61$

$<.119 E+C$

$<.972 E-01$
BETA

(CI)

$<.656 F-02$

$<.562 E-21$

$<.74$ E F-C2

$.799 E-01$

$.543 E-12$

$<.578 E-12$

$.120 \mathrm{E}-01$

. $291 E-r 1$

$.573 E-01$
$S R-93$

(CI)

e.796F-04

- $148 F-0$ ?

<. 29? F-D 4

<. 241E-02

$<.127 E-02$

<.305E-02

$.9575-02$

- $903 E-02$
$R U-116$

(CI)

$<.175 E-63$

<. 223E-01

<.620F-O4

$<.17$ BE-DI

*.2z9E- 22

SEP

NOV

DEC

ADJ

73 SUM

PRE SUM

- 113E+10

- $156 F+1 ?$

$.157 \mathrm{E}+12$

73 DERAY - 113F+10

PREDERAY - 156F+12

TOTIFCAY $.157 F+12$

$<.379 F+50$

$.422 E+C 3$

. $423 F+C 3$

$<.270 F+90$

- $227 F+106$

$.2 ? 75+15$

<.269F-01

- $482 E+13$

2. 427E-01

$<.741 E+33$

$.482 E+03<.741 E+03$

<. $370 F+50$

$.422 E+13$

<.?38E+00

$.325 E+4$

<.26RE-71

- $3995+13$

$<.296 F-1$

$.4 P 3 F+D 3$

$.3 ? 5 F+94$

$.389 E+03$

<. $128 E+D 2$

$<.471 E-01$

. $361 E+C 3$

$.361 E+03$

$<.308 E-01$

$<.440 E+02$

$<.440 E+02$

$<.468 E-01$

$.297 E+03$

$.297 E+03$

$<.289 E-01$

$<.218 E+32$

$<.218 E+02$

(UTT/ML)

JUL
$<. ? 4>E-.7$

(UCI/ML)

$A P R$

(UC,T/ML)

JUL

(UCI/ML) $A P R$

$.2825-55$

$<.734 E-07$

$<.449 F-C 6$

(UCI/ML)

$\triangle P R$

$.428 E-3 E$

MUCI

$<.133 E-\square 5$

$<.124 E+02$
$.574 E+06$

$.574 E+06$

$<.124 E+02$

$.574 E+06$

$.574 E+06$

$<.482 E+03$

$<.482 E+13$

$<.492 E+03$

$<.482 E+03$

(UCI/ML)

SEP
C. 96 RE-0?
$U-233$

(GM)

\section{AVERAGF}

CONC

$<.2 R^{2} 5 F-C$

$<.238 E-65$

$<.237 F-07$

$<.377 E-n 7$

$<.41 F E-07$

$<.272 E-07<.364 E-08$ 
MONTHLY SUMMARY OF LIOUID WASTF DISCHARGED TO GROUNO FROM $1 / 1$ TO $9 / 301973$ AT DISPOSAL SITE $216-A-? 5$ PONC WASTE STREAM DF SCPIPTION- AR VAULT COOLING WATER SINCE JANUARY 1,1969.

\begin{tabular}{|c|c|c|c|c|c|c|c|c|}
\hline 1973 & $\begin{array}{l}\text { VOLUME } \\
\text { (L) }\end{array}$ & $\begin{array}{l}P U \\
(G M)\end{array}$ & $\begin{array}{l}\text { BETA } \\
\text { (CI) }\end{array}$ & $\begin{array}{l}S R-90 \\
(C I)\end{array}$ & $\begin{array}{l}R U-106 \\
(C I)\end{array}$ & $\begin{array}{l}\text { CS }-137 \\
(\mathrm{CI})\end{array}$ & $\begin{array}{l}\mathrm{CO}-60 \\
\text { (CI) }\end{array}$ & $\begin{array}{l}U \\
(K G M)\end{array}$ \\
\hline $\begin{array}{l}\text { JAN } \\
\text { FEB } \\
\text { MAR } \\
\text { APR } \\
\text { MAY } \\
\text { JUN } \\
\text { JUL } \\
\text { AUG } \\
\text { SEP } \\
\text { OCT } \\
\text { NOY } \\
\text { DEC } \\
\text { ADJ }\end{array}$ & $\begin{array}{l}.757 E+07 \\
.757 F+77 \\
.757 F+07 \\
.757 F+07 \\
.7575+27 \\
.946 E+07 \\
.757 E+07 \\
.259+18 \\
.395 E+18\end{array}$ & $\begin{array}{r}<.191 F-04 \\
.844 E-04 \\
.996 E-34 \\
<.924 E-04 \\
.496 E-3 \\
.325 E-13 \\
.494 E-04 \\
<.52 E E-04 \\
.116 E-13\end{array}$ & $\begin{array}{r}<.266 E-03 \\
<.378 E-03 \\
<.234 E-23 \\
.422 E-3 \\
.510 E-3 \\
.915 E-13 \\
.382 E-13 \\
<.103 E-.2 \\
.786 F-02\end{array}$ & & & & & $\begin{array}{l}<.768 E-01 \\
<.768 E-01 \\
<.768 E-01 \\
<.768 E-01 \\
<.750 E-01 \\
<.972 E-01 \\
<.802 E-01 \\
<.276 E+00 \\
<.308 E+00\end{array}$ \\
\hline $\begin{array}{l}73 \text { DECAY } \\
\text { PREDERAY } \\
\text { TOTDECAY }\end{array}$ & $\begin{array}{l}.111=+9 \\
.211 F+15 \\
.222 E+1 C\end{array}$ & $\begin{array}{l}<.132 F-2 \\
<.656 F+2 \\
<.658 F+0 C\end{array}$ & $\begin{array}{l}<.132 E-11 \\
<.368 E+30 \\
<.378 E+10\end{array}$ & $\begin{array}{l}<.441 E-11 \\
<.441 E-01\end{array}$ & $\begin{array}{l}<.123=-01 \\
<.123 E-01\end{array}$ & $\begin{array}{l}<.789 E-01 \\
<.789 E-01\end{array}$ & $\begin{array}{l}<.223 E-01 \\
<.223 E-01\end{array}$ & $\begin{array}{l}<.114 E+01 \\
<.449 E+01 \\
<.564 E+01\end{array}$ \\
\hline
\end{tabular}

\section{MONTHLY}

MAXTMUM

CONC

AVERAGE

CONC

$\begin{array}{ll}\text { (UCT/ML) } & \text { (UCT/ML) (UCT/ML) (UCI/ML) (UCT/ML) (UCI/ML) } \\ M A Y & \text { SEF } \\ .4 E P F-98 & .258 F-G 5 \\ & \\ <.731 F-09 & <.109 E-86\end{array}$

\author{
(UCI/ML) \\ AUG \\ C. 355E- 08 \\ c. $342 E-38$
}


MONTHLY SUMMARY OF LIQUID WASTE DISCHARGED TO GROUND FROM 1/1 TO $9 / 301973$ AT

DI SPOSAL STTF 215-8-3

WASTE STREAM DESCPIPTION-
SUMMARY

9 PLANT COOLING WATEQ AND CHEMICAL SEWER, CR VAULT COOLING WATER, PUREX CHEYICAL SEWEP, ITS-1 AND ITS-2 COOLING WATER.

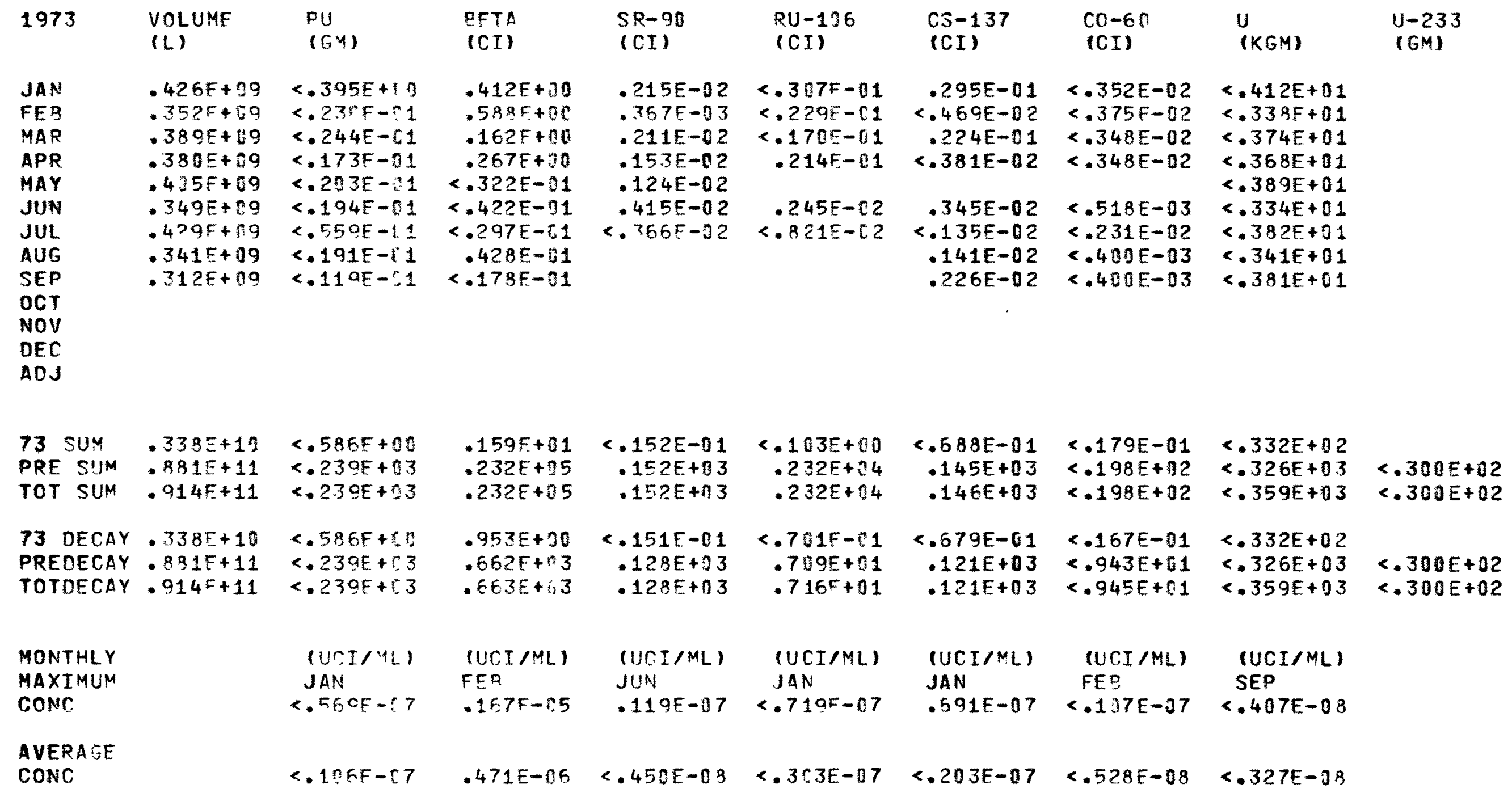

OCT

NOV

DEC

$A D J$

73 SUY TOTE SJM $\triangle 891 E+11$

73 DECAY $338 F+10$

\section{PREDECAY . 831E+11}

TOTDECAY $.914 F+11$

$<.586 E+00$ <. $230 E+03$

<. $239 E+23$

<.586F+r.

$<.239 E+3$

$<.239 E+3$

$.159 F+01$ $.232 E+05$

$.232 E+\square 5$

$.152 E-01$
$.152 E+03$
$.152 E+13$

<. $103 E+00$ - $232 E+? 4$ - $232 E+04$

<.588E-01 $.145 E+03$

$.146 E+03$

$.953 E+30$ $.562 E+13$

<.151E-01 $-128 E+23$

$<.701 F-1$ .7 QE +01

$<.679 E-01$

$.121 E+03$

- $263 E+3$

$-128 E+03$

$.716 F+01$

-121E+03

C.179E-01

$<.198 E+122$

$+.198 E+02$

R.167E-01

$<.943 E+61$

$<.945 E+01$

\section{(UCI/1L)}

JAN

$<.56$ CF $-: 27$

(UCI I ML)

FER

(UCI/ML)$$
\text { JUN }
$$

(UCI/ML)

$.1 E 7 F-85$

$.119 E-07<.719 F-07$

(UCI/ML)

JAN
$.591 E-07$

(UCI/ML)
FEP

$<.137 E-37$

$<.332 E+02$
$<.326 E+03$

<. 359E+03

c. $332 E+02$

$<.326 E+03$

<.359E+ 03

$<.300 E+02$
$<.300 E+02$

\section{A VERAGE}

CONC

(UCI/ML) SEP $<.407 E-08$

$<.327 E-3 B$

$<.300 E+02$ $<.300 E+02$ 
MONTHLY SUMMARY DF LIQUTO WASTE IISCHARGEO TO GROUNO FROM $1 / 1$ TO $9 / 33^{1973}$ AT OISPOSAL SITF $216-B-3$ POAE

WASTE STREAM NESCRICTION- R PLANT COOLING WATER, SINCE JANUARY $1,1968$.

\begin{tabular}{|c|c|c|c|c|c|c|c|c|}
\hline 1973 & $\begin{array}{l}\text { VOLUME } \\
\text { (L) }\end{array}$ & $\begin{array}{l}P U \\
(G M)\end{array}$ & $\begin{array}{l}\text { EETA } \\
\text { (CI) }\end{array}$ & $\begin{array}{l}S R-90 \\
\text { (CI) }\end{array}$ & $\begin{array}{l}R U-106 \\
(C I)\end{array}$ & $\begin{array}{l}C S-137 \\
(C I)\end{array}$ & $\begin{array}{l}C O-60 \\
\text { (CI) }\end{array}$ & $\begin{array}{l}U \\
(K G M)\end{array}$ \\
\hline $\begin{array}{l}\text { JAN } \\
\text { FEB } \\
\text { MAP } \\
\text { APR } \\
\text { MAY } \\
\text { JUN } \\
\text { JUL } \\
\text { AUG } \\
\text { SEP } \\
\text { OCT } \\
\text { NOV } \\
\text { DEC } \\
\text { ADJ }\end{array}$ & 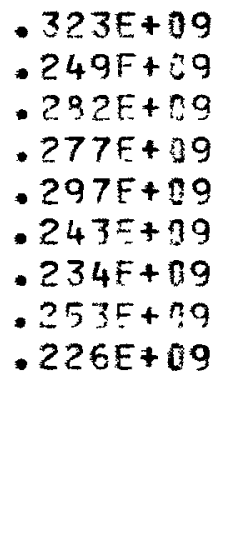 & $\begin{array}{r}<.612 E-13 \\
.507 E-33 \\
<.177 E-92 \\
<.28 f E-22 \\
.677 E-92 \\
<.447 E-02 \\
.774 E-03 \\
<.335 E-32 \\
<.705 E-04\end{array}$ & $\begin{array}{r}.361 E+C 0 \\
.575 E+00 \\
.595 E-01 \\
.261 F+0 \\
<.25 C E-C 1 \\
.208 E-01 \\
<.118 E-01 \\
.334 E-71 \\
.986 E-02\end{array}$ & & & & & $\begin{array}{l}\text { e. } 325 E+01 \\
\text { e. } 251 E+01 \\
\text { e. } 284 E+01 \\
\text { e. } 280 E+01 \\
\text { e. } 298 E+01 \\
\text { e. } 245 E+01 \\
\text { e. } 248 E+01 \\
\text { e. } 268 E+01 \\
\text { e. } 228 E+01\end{array}$ \\
\hline $\begin{array}{l}73 \text { SUM } \\
\text { PRE SUM } \\
\text { TOT SUM }\end{array}$ & $\begin{array}{l}.239 E+10 \\
.105 E+11 \\
.129 F+11\end{array}$ & $\begin{array}{l}<.205 E-01 \\
<.792 E+01 \\
<.795 E+01\end{array}$ & $\begin{array}{l}.136 E+01 \\
.720 E+02 \\
.734 E+32\end{array}$ & $\begin{array}{l}<.250 E+01 \\
<.250 E+01\end{array}$ & $\begin{array}{l}\text { e. } 389 E+01 \\
\text { e.389E+31 }\end{array}$ & $\begin{array}{l}<.680 E+01 \\
<.580 E+01\end{array}$ & $\begin{array}{l}.375 E+01 \\
.375 E+01\end{array}$ & $\begin{array}{l}<.243 E+02 \\
<.304 E+02 \\
<.547 E+02\end{array}$ \\
\hline $\begin{array}{l}73 \text { NFCAY } \\
\text { PREOECAY } \\
\text { TOTDECAY }\end{array}$ & $\begin{array}{l}\cdot 233 E+19 \\
.105 E+11 \\
.120 E+11\end{array}$ & $\begin{array}{l}<.275 E-01 \\
<.793 E+81 \\
<.795 E+01\end{array}$ & $\begin{array}{l}.711 E+00 \\
.213 E+02 \\
.220 E+02\end{array}$ & $\begin{array}{l}<.223 E+01 \\
<.223 E+01\end{array}$ & $\begin{array}{l}<.121 E+00 \\
<.121 E+00\end{array}$ & $\begin{array}{l}<.612 E+01 \\
<.612 E+01\end{array}$ & $\begin{array}{l}18 B E+11 \\
.188 E+01\end{array}$ & $\begin{array}{l}<.243 E+02 \\
<.304 E+02 \\
<.547 E+02\end{array}$ \\
\hline $\begin{array}{l}\text { MONTHLY } \\
\text { MAXIMUM } \\
\text { CONC }\end{array}$ & & $\begin{array}{l}(U C I / M L) \\
\text { MAY } \\
.14: F-C 8\end{array}$ & $\begin{array}{l}(U C I / M L) \\
\text { FER } \\
.231 E-[5\end{array}$ & $(1 j r \mathrm{~T} / M L)$ & (UCI/ML) & (UCI/ML) & (UCI /ML) & $\begin{array}{l}\text { (UCI/ML) } \\
\text { AUG } \\
<.354 E-09\end{array}$ \\
\hline $\begin{array}{l}\text { AVERACF } \\
\text { CONC }\end{array}$ & & $<.528 E-69$ & $.571 F-06$ & & & & & $<.339 E-03$ \\
\hline
\end{tabular}


MONTHLY SUMMARY OF LIQUID WASTE DISCHARGFD TO GROUND FROM $1 / 1$ TO $9 / 301973$ AT DISPOSAL SITF $216-3-3$ PONC

WASTE STRFAM DESCPIFTION- IN-TANK SOLIDIFICATION NO. 1 CCOLING WATER, SINCE JANUARY 1,1969.

\begin{tabular}{|c|c|c|c|c|c|c|c|c|}
\hline 1973 & $\begin{array}{l}\text { VOLUME } \\
\text { (L) }\end{array}$ & $\begin{array}{l}P U \\
\text { (GM) }\end{array}$ & $\begin{array}{l}\text { EETA } \\
\text { (CI) }\end{array}$ & $\begin{array}{l}S R-90 \\
(C I)\end{array}$ & $\begin{array}{l}R U-136 \\
\text { (CI) }\end{array}$ & $\begin{array}{l}C S-137 \\
\text { (CI) }\end{array}$ & $\begin{array}{l}(0-60 \\
\text { (CI) }\end{array}$ & $\begin{array}{l}U \\
\text { (KGM) }\end{array}$ \\
\hline $\begin{array}{l}\text { JAN } \\
\text { FEB } \\
\text { MAR } \\
\text { APR } \\
\text { MAY } \\
\text { JUN } \\
\text { JUL } \\
\text { AUG } \\
\text { SEP } \\
\text { OCT } \\
\text { NOV } \\
\text { OEC } \\
\text { ADJ }\end{array}$ & $\begin{array}{l}\cdot 322 E+18 \\
.303 F+08 \\
.322 F+78 \\
.313 E+68 \\
.338 E+08 \\
.327 F+08 \\
.132 E+08 \\
.132 E+18 \\
.132 E+08\end{array}$ & $\begin{array}{r}.157 F-83 \\
<.160 E-82 \\
<.577 F-34 \\
.42 F F-03 \\
.150 E-83 \\
<.772 E-83 \\
.313 E-83 \\
.225 E-83 \\
.623 E-85\end{array}$ & $\begin{array}{r}.328 F-82 \\
<.141 E-12 \\
<.573 E-.22 \\
.182 E-92 \\
<.163 E-02 \\
<.464 E-02 \\
<.164 E-82 \\
.356 E-02 \\
.100 E-12\end{array}$ & & & & & $\begin{array}{l}<.325 E+00 \\
<.307 E+00 \\
<.325 E+30 \\
<.308 E+00 \\
<.339 E+00 \\
<.330 E+00 \\
<.134 E+00 \\
<.141 E+00 \\
<.134 E+00\end{array}$ \\
\hline $\begin{array}{l}73 \text { SUM } \\
\text { PRE SUM } \\
\text { TOT SUM }\end{array}$ & $\begin{array}{l}.231 E+C 9 \\
.139 E+10 \\
.163 E+10\end{array}$ & $\begin{array}{l}<.369 F-12 \\
<.695 E+00 \\
e .697 F+00\end{array}$ & $\begin{array}{l}<.241 E-61 \\
<.166 F+01 \\
<.169 E+01\end{array}$ & $\begin{array}{l}<.343 E-71 \\
<.343 E-01\end{array}$ & $\begin{array}{l}<.890 E-01 \\
\text { e. } 8.80 F-01\end{array}$ & $\begin{array}{l}.431 F+00 \\
.431 E+00\end{array}$ & $\begin{array}{l}<.160 E-71 \\
<.160 E-01\end{array}$ & $\begin{array}{l}<.234 E+01 \\
<.541 E+01 \\
<.775 E+11\end{array}$ \\
\hline $\begin{array}{l}73 \text { DECAY } \\
\text { PREDECAY } \\
\text { TOTOECAY }\end{array}$ & $\begin{array}{l}.231 E+.9 \\
.139 E+10 \\
.163 E+18\end{array}$ & $\begin{array}{l}<.350 F-12 \\
<.69 C E+71 \\
<.693 F+10\end{array}$ & $\begin{array}{l}<.152 E-01 \\
<.915 E+00 \\
<.920 E+00\end{array}$ & $\begin{array}{l}<.311 E-C 1 \\
\text { e. } 311 E-01\end{array}$ & $\begin{array}{l}<.6 C 2 E-C 2 \\
<.532 E-02\end{array}$ & $\begin{array}{l}.399 E+00 \\
.399 E+100\end{array}$ & $\begin{array}{l}<.914 E-02 \\
<.914 E-02\end{array}$ & $\begin{array}{l}<.234 E+01 \\
<.541 E+01 \\
<.775 E+11\end{array}$ \\
\hline $\begin{array}{l}\text { MONTHLY } \\
\text { MAXIMUY } \\
\text { CONC }\end{array}$ & & $\begin{array}{l}(U C I / M L) \\
F E O \\
<.324 F-9\end{array}$ & $\begin{array}{l}(U C I / M L) \\
\text { AUF } \\
.264 E-06\end{array}$ & (UCI/ML) & (UCI/ML) & $(U C I / M L)$ & (UCI / ML) & $\begin{array}{l}\text { (UCI/ML) } \\
\text { AUG } \\
\times .354 E-08\end{array}$ \\
\hline $\begin{array}{l}\text { AVFRATE } \\
\text { CONC }\end{array}$ & & $<.981 F-19$ & $<.134 E-06$ & & & & & $<.337 E-18$ \\
\hline
\end{tabular}


MONTHLY SUMMARY OF LIQUID WASTE DISCHARGED TO GROUND FROM $1 / 1$ TO $9 / 301973$ AT DISPCSAL. SITE $216-\mathrm{B}-3$ PONC WASTF STREAM FESCRIPTION- IN-TANK SOLIOIFICATION NO, 2 CCOLING WATER, SINCE JANUARY 1 , 1959.

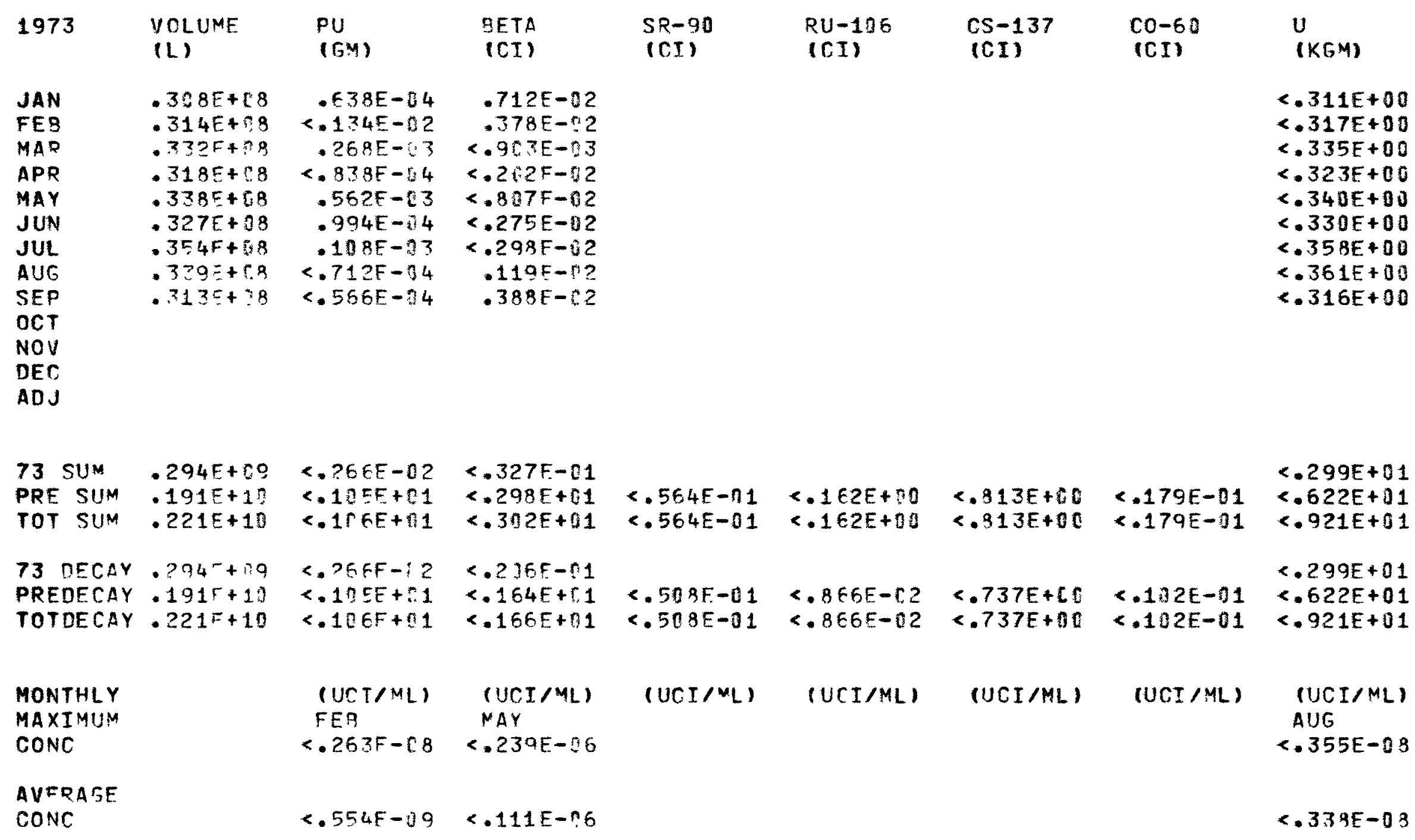


$\stackrel{0}{\frac{w}{0}}$

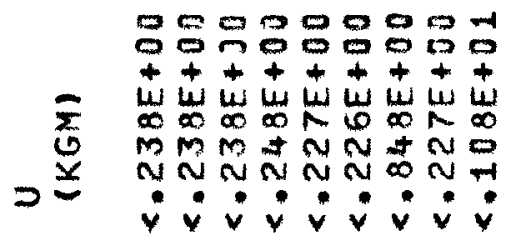

NNNN

@o 0

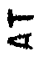

$\stackrel{M}{0}$

매山心

P= NR $N^{\infty}$

엉요

m? $m$

$m \cong m M$

i 9

山心

$\rightarrow-198$

in

ให?

$\overbrace{0}^{2}$

ने

욘

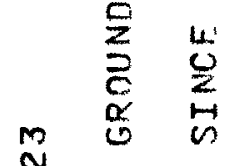

N

$\circ \dot{0}$

感

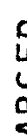

C

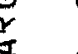
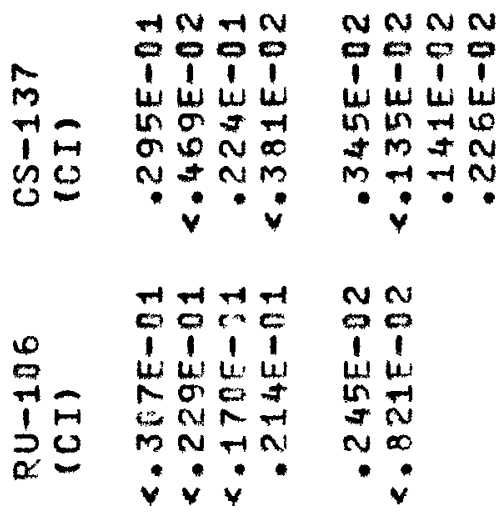

NMNNNNN

0090000

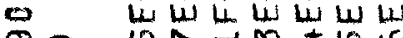

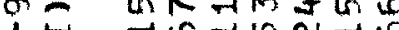

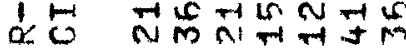

$n=\cdots \cdot \cdot$

壱

$\leftrightarrow$

$\operatorname{tin}$

$\varangle<4$

$\times 0 \%$

a.

严

$\overbrace{\rightarrow \rightarrow 2}$

- 1.

415

co.

त्-

a

$\sum \leftarrow$

至出

in $\frac{\Sigma}{\alpha}$

$>\frac{1}{2}$

至为告

I은

은

을벙는

$\frac{4}{3}$

9

$+1,9,95 \%$

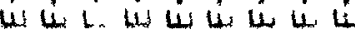

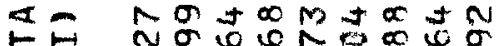

uU $\rightarrow$ in $\infty$ in $N$ N

a $=?$ ? ? ? ? ?

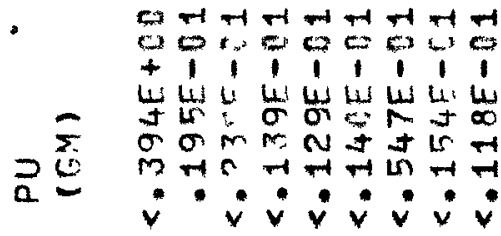

$\infty \infty \infty \infty \infty \sigma \infty \infty$

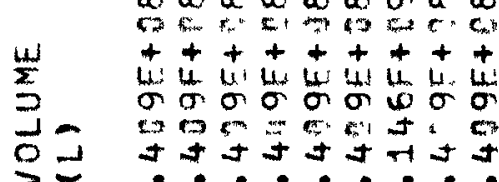

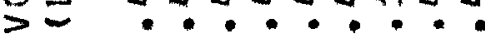

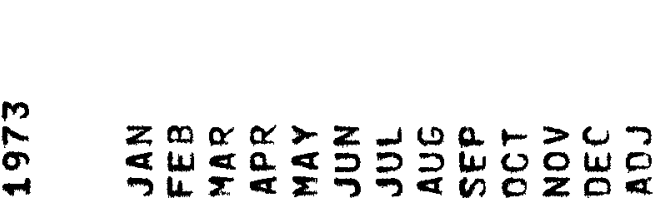

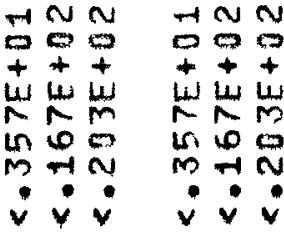

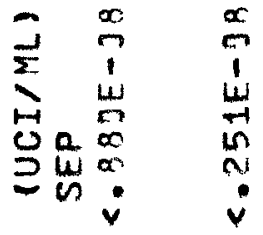

당항

山े

W世

g.

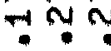

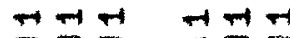

$+1 \rightarrow \infty$

$1+$

4. 네

क是是

는?

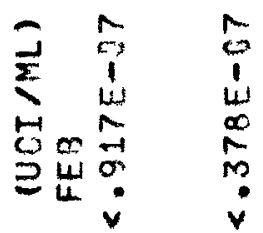

00 $1+$

$\infty$ in

$\infty$ 車

$\infty$

$0 \pm$

$1 \div$

$4 \omega$

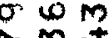

$N M \pm$

$\infty \div$

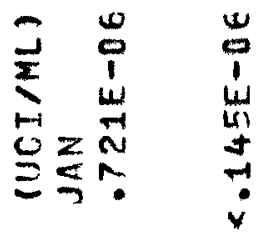

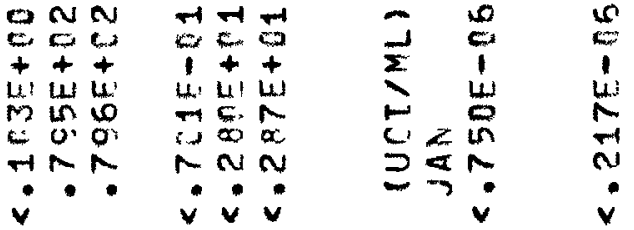

D車

Li:

Nis

in

$\therefore$.

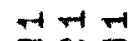

$=0$

WU

$\rightarrow \infty e$

is in in

$M N M$

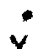

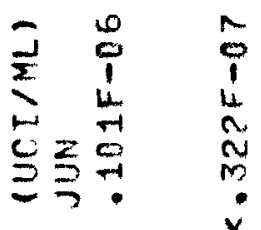

$\triangle M M \because N O$

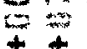

$++$

4. 4.4

Non

$M N$

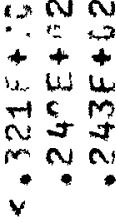

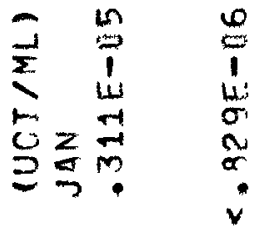

$\rightarrow N N=N N$

$=0 \mathrm{~s}, 4$

$+++1+1$

thu 4 Uा

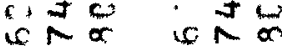

Lit

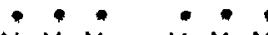

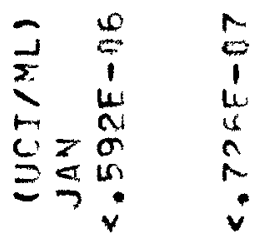

ac $\Rightarrow \pi+\infty$

$0+1+1+1$

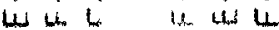

$m \rightarrow \sigma \quad m$ o

$\wedge \infty N \quad N \infty$

t)

$5>2>$

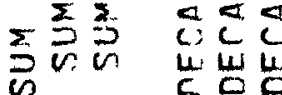

$\omega \leftarrow$

$c$.

$m \propto O$

$m \alpha O$

a

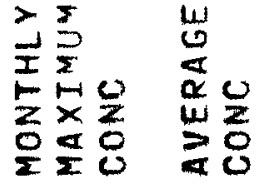


MONTHLY SUMMARY OF LIQUID WASTF DISCHARGEO TO GROUND FROM $1 / 1$ TO $9 / 391973$ AT DISPOSAL STTF 216- $2-63$ SUMMAOY WASTE STREAM TFSCRIPTION- R PLANT CHEMICAL SFWEO, B PLANT COOLING WATER ANC ITS-2 COOLING WATER

\begin{tabular}{|c|c|c|c|c|c|c|c|c|}
\hline 1973 & $\begin{array}{l}\text { VOLUME } \\
\text { (L) }\end{array}$ & $\begin{array}{l}F U \\
(G M)\end{array}$ & $\begin{array}{l}\text { PETA } \\
\text { (CI) }\end{array}$ & $\begin{array}{l}5 R-90 \\
\text { (CI) }\end{array}$ & $\begin{array}{l}R U-136 \\
(C I)\end{array}$ & $\begin{array}{l}\mathrm{CS}-137 \\
\text { (CI) }\end{array}$ & $\begin{array}{l}(0-60) \\
\text { (CI) }\end{array}$ & $\begin{array}{l}U \\
(K G M)\end{array}$ \\
\hline $\begin{array}{l}\text { JAN } \\
\text { FEB } \\
\text { MAR } \\
\text { APR }\end{array}$ & $\begin{array}{r}.333 E+18 \\
.235 E+28 \\
.259 E+18 \\
435 F+18\end{array}$ & $\begin{array}{l}<.686 E-02 \\
<.344 E-02 \\
<.416 E-62 \\
<.731 F-?\end{array}$ & $\begin{array}{r}.248 F-81 \\
<.484 E-01 \\
.573 F+r C \\
.245 F-C 1\end{array}$ & $.146 E-01$ & $\cdot 1 E 1 F-C_{2}$ & $.439 E-01$ & & $\begin{array}{l}<.134 E+00 \\
<.137 E+00 \\
<.203 E+30 \\
<.258 F+00\end{array}$ \\
\hline $\begin{array}{l}\text { APR } \\
\text { MAY } \\
\text { JUN } \\
\text { JUL } \\
\text { AUE } \\
\text { SEP }\end{array}$ & $\begin{array}{l}.425 E+88 \\
.676 E+08 \\
.785 E+88 \\
.845 E+88 \\
.814 E+88 \\
.739 E+88\end{array}$ & $\begin{array}{l}<.731 E-2 \\
<.843 E-3 \\
<.338 E-11 \\
<.145 E-11 \\
<.77 .5 E-.2 \\
<.859 E-12\end{array}$ & $\begin{array}{r}.246 E-61 \\
.207 E-01 \\
=.236 F-11 \\
<.738 F-11 \\
<.175 E+.0 \\
.297 F-11\end{array}$ & & $=$ & & $<.754 E-02$ & $\begin{array}{l}<.25 A E+00 \\
<.375 E+00 \\
<.498 E+00 \\
<.491 E-01 \\
<.437 E+00 \\
<.410 E+00\end{array}$ \\
\hline
\end{tabular}

OCT

NOV

DEC

ADJ

73 SUM

PRE SUM

TOT SUM

$.518=+\cdots 9$ $.8115+09$

$.1325+17$

73 DESAY . 518E+:9 PREDECAY REIF+29 TOTDECAY . 132E+10

MONTHLY

MAXIMUM

CONC

AVEPACE CONG
<. $948 E-1$ <. $121 E+00$

<. $21 E E+?$

$<048 F-1$

$<.121 E+C 0$

<. $21 F E+0$

$<.940 E+10$

<.748F+01

$<.842 E+01$

$<.524 F+10$

$<.474 E+i 1$

$<.536 E+1$

\section{$146 F-01$}

$.165 E+01$

$.167 E+01$

- $1 E 1 F-02$

$<.138 F+00$

$<.1395+00$

$.144 E-01$

$.153 \mathrm{E}+01$

$.154 F+01$

$.111=-2$

$<.282 E-11$

$<.293 F-1$

$.439 F-01$

$<.625 E+10$

$<.669 \mathrm{~F}+10$

$<.754 E-32$

$<.600 E-02$

$<.135 F-01$

<. $256 E+11$

$<.436 E+31$

$<.691 E+31$

$.434 E-01$

$<.589 E+00$

$<.717 E-02$

$<.409 E-02$

$<.255 E+91$

C. $632 E+0 \mathrm{C}$

$<.113 E-01$

c. $436 E+01$

$\times .691 E+01$

(Ur,I/ML)
JUN
$<. ? 64 F-07$

$\begin{array}{ll}\text { (UCI/ML) } & \text { (UCI/ML) } \\ \text { MAR } & \text { MAR } \\ .163 E-04 & .4[7 F-06\end{array}$

(UCI/ML)

MAF

$.449 E-67$

(UCI/ML)

MAR

.12 JE-05

(UCI /ML)

$<.111 E-05$

(UCI/ML)

JUN

$<.211 E-08$

$<.112 E-07<.131 E-55 \quad .231 E-97 \quad .311 E-68 \quad .348 E-07 \quad<.145 E-07 \quad<.164 E-08$ 
MONTHLY SUMMARY OF LIQUID WASTF DISCHARGED TO GROUND FRON $1 / 1$ TO $9 / 301973$ AT DISPOSAL STTE 216-E-E3 TREASH WASTE STREAM DFSCRIFTIOA- 9 PLANT CHFMICAL SEWER FROM MAY 7, 1970 TO DATF.

\begin{tabular}{|c|c|c|c|c|c|c|c|c|}
\hline 1973 & $\begin{array}{l}\text { VOLUME } \\
\text { (L) }\end{array}$ & $\begin{array}{l}P U \\
(G M)\end{array}$ & $\begin{array}{l}\text { BETA } \\
\text { (CI) }\end{array}$ & $\begin{array}{l}S R-90 \\
\text { (CI) }\end{array}$ & $\begin{array}{l}R U-106 \\
(C I)\end{array}$ & $\begin{array}{l}C S-137 \\
(C I)\end{array}$ & $\begin{array}{l}\mathrm{CO}-60 \\
\text { (CI) }\end{array}$ & $\begin{array}{l}U \\
\text { (KGM) }\end{array}$ \\
\hline $\begin{array}{l}\text { JAN } \\
\text { FEB } \\
\text { MAR } \\
\text { APR } \\
\text { MAY } \\
\text { JUN } \\
\text { JUL } \\
\text { AUG } \\
\text { SEP } \\
\text { OCT } \\
\text { NOU } \\
\text { DEC } \\
\text { ADJ }\end{array}$ & $\begin{array}{l}.393 E+08 \\
.235 F+38 \\
.352 E+18 \\
.425 E+88 \\
.676 E+78 \\
.795 E+18 \\
.845 E+08 \\
.814 F+18 \\
.739 E+08\end{array}$ & $\begin{array}{l}<.686 E-02 \\
<.344 E-C 2 \\
<.41 E E-: 2 \\
<.731 E-C 2 \\
<.843 E-C 2 \\
<.338 E-[1 \\
<.14 E E-81 \\
<.77 . E-82 \\
<.859 E-12\end{array}$ & $\begin{array}{r}.248 E-01 \\
<.484 E-01 \\
.573 E+7 \\
.246 E-01 \\
.297 E-81 \\
<.236 E-01 \\
<.708 E-11 \\
<.125 E+00 \\
.297 E-01\end{array}$ & $.14 E E-D 1$ & $\cdot 1 E 1 E-12$ & $.439 E-01$ & $<.754 E-02$ & $\begin{array}{l}<.184 E+00 \\
<.137 E+00 \\
<.208 E+00 \\
<.259 E+00 \\
<.375 E+00 \\
<.499 E+0 D \\
<.491 E-01 \\
<.437 E+30 \\
<.410 E+00\end{array}$ \\
\hline $\begin{array}{l}73 \text { SUM } \\
\text { PRE SUM } \\
\text { TOT SUM }\end{array}$ & $\begin{array}{l}.518 E+9 \\
.655 F+19 \\
.117 E+19\end{array}$ & $\begin{array}{l}<.04 R F-21 \\
<.90 .2 F-61 \\
<.185 F+8\end{array}$ & $\begin{array}{l}<.940 E+90 \\
<.756 E+1 \\
<.855 F+1\end{array}$ & $\begin{array}{l}.146 E-01 \\
.165 E+C 1 \\
.157 E+01\end{array}$ & $\begin{array}{r}.161 E-02 \\
<.140 E+00 \\
<.142 E+00\end{array}$ & $\begin{array}{r}.439 E-01 \\
<.622 E+10 \\
<.665 E+00\end{array}$ & $\begin{array}{l}<.754 E-02 \\
<.595 E-02 \\
<.134 E-01\end{array}$ & $\begin{array}{l}<.256 E+01 \\
<.329 E+01 \\
<.584 E+01\end{array}$ \\
\hline $\begin{array}{l}73 \text { DECAY } \\
\text { PREDECAY } \\
\text { TOTDECAY }\end{array}$ & $\begin{array}{l}.518 F+59 \\
.655 E+39 \\
.117 F+15\end{array}$ & $\begin{array}{l}<.949 F-91 \\
<.905 E-31 \\
<.185 F+C 0\end{array}$ & $\begin{array}{l}<.524 F+10 \\
<.475 E+11 \\
<.533 F+.1\end{array}$ & $\begin{array}{l}\cdot 144 E-C 1 \\
.153 F+01 \\
.155 F+31\end{array}$ & $\begin{array}{r}.111 F-02 \\
<.285 F-81 \\
<.296 F-01\end{array}$ & $\begin{array}{r}.434 E-01 \\
<.585 E+00 \\
<.629 E+00\end{array}$ & $\begin{array}{l}<.717 E-0 ? \\
<.394 E-02 \\
<.111 E-01\end{array}$ & $\begin{array}{l}r .255 E+01 \\
<.329 E+01 \\
<.594 E+01\end{array}$ \\
\hline $\begin{array}{l}\text { MONTHLY } \\
\text { MAXIMUM } \\
\text { CONC }\end{array}$ & & $\begin{array}{l}(U C I / M L) \\
\text { JUN } \\
<.264 F-i 7\end{array}$ & $\begin{array}{l}(U C I / M L) \\
\text { MAR } \\
.16^{*} F-C 4\end{array}$ & $\begin{array}{l}\text { (UCI/ML) } \\
M A R \\
.407 E-06\end{array}$ & $\begin{array}{l}\text { (UCI/ML) } \\
\text { YAR } \\
.449 E-07\end{array}$ & $\begin{array}{l}\text { (UCI/ML) } \\
\text { MAR } \\
.123 E-05\end{array}$ & $\begin{array}{l}\text { (UGI/ML) } \\
\text { MAY } \\
\text { <.111E-36 }\end{array}$ & $\begin{array}{l}\text { (UCI/ML) } \\
\text { JUN } \\
<=211 E-08\end{array}$ \\
\hline $\begin{array}{l}\text { AVERAGE } \\
\text { CONC }\end{array}$ & & $<.112 F-7$ & e.181F-?5 & $.281 E-37$ & $.311 F-98$ & $.348 E-07$ & $<.145 E-07$ & $<.164 E-08$ \\
\hline
\end{tabular}


MONTHLY SUMMARY OF LIQUID WASTE DISCHARGED TO GROUND FROM $1 / 1$ TO $9 / 301973$ AT BISPOSAL SITF 216-S-11 PONC WASTE STREAM DESCPIPTION- 202-S CHEYICAL SFWF?

\begin{tabular}{|c|c|c|c|c|c|c|c|c|}
\hline 1973 & $\begin{array}{l}\text { VOLUME } \\
\text { (L) }\end{array}$ & $\begin{array}{l}P U \\
(G M)\end{array}$ & $\begin{array}{l}\text { RETA } \\
\text { (CI) }\end{array}$ & $\begin{array}{l}S R-90 \\
\text { (CI) }\end{array}$ & $\begin{array}{l}R U-106 \\
(C I)\end{array}$ & $\begin{array}{l}\mathrm{CS}-137 \\
(\mathrm{CI})\end{array}$ & $\begin{array}{l}\mathrm{CO}-60 \\
\text { (CI) }\end{array}$ & $U_{\text {(KGM) }}$ \\
\hline $\begin{array}{l}\text { JAN } \\
\text { FEB } \\
\text { MAR } \\
\text { APR } \\
\text { MAY } \\
\text { JUN } \\
\text { JUL } \\
\text { AUG } \\
\text { SEP } \\
\text { OCT } \\
\text { NOY } \\
\text { DEC } \\
\text { ADJ }\end{array}$ & $\begin{array}{l}.169 E+78 \\
.153 E+18 \\
.169 E+78 \\
.164 E+78 \\
.169 E+78 \\
.164 E+88 \\
.169 E+78 \\
.159 E+88 \\
.164 E+78\end{array}$ & $\begin{array}{r}.134 E-3 \\
<.358 E-14 \\
.12 E E-3 \\
<.562 E-4 \\
<.312 E-32 \\
.355 E-3 \\
<.482 E-34 \\
.777 E-4 \\
<.422 E-14\end{array}$ & $\begin{array}{r}.598 E-03 \\
<.818 E-03 \\
<.345 E-73 \\
<.597 E-13 \\
.158 E-52 \\
<.107 E-02 \\
<.540 E-33 \\
.519 E-83 \\
<.812 E-03\end{array}$ & & & & & $\begin{array}{l}<.171 F+00 \\
<.155 E+00 \\
<.171 E+00 \\
<.168 E+00 \\
<.17 D E+00 \\
<.165 E+00 \\
<.179 E+00 \\
<.179 E+00 \\
<.165 E+00\end{array}$ \\
\hline $\begin{array}{l}73 \text { SUM } \\
\text { PRE SUM } \\
\text { TOT SUM }\end{array}$ & $\begin{array}{l}140 F+=9 \\
372 E+10 \\
.317 F+10\end{array}$ & $\begin{array}{l}<.398 E-12 \\
<.224 E+30 \\
<.228 F+00\end{array}$ & $\begin{array}{r}.695 E-02 \\
.101 F+03 \\
.101 F+03\end{array}$ & $\begin{array}{l}-111 E+01 \\
.111 E+01\end{array}$ & $\begin{array}{l}.101 F+2 \\
.101 F+02\end{array}$ & $\begin{array}{l}-111 E+01 \\
.111 E+01\end{array}$ & $\begin{array}{l}<.133 E+00 \\
<.133 E+00\end{array}$ & $\begin{array}{l}<.152 E+01 \\
<.249 E+02 \\
<.265 E+02\end{array}$ \\
\hline $\begin{array}{l}73 \text { DERAY } \\
\text { PREDECAY } \\
\text { TOTDECAY }\end{array}$ & $\begin{array}{l}149 E+9 \\
.302 E+10 \\
.317 E+1 C\end{array}$ & $\begin{array}{l}<.398 F-.2 \\
<.224 E+5 \\
<.228 E+30\end{array}$ & $\begin{array}{r}<.459 E-02 \\
.372 E+01 \\
.372 E+01\end{array}$ & $\begin{array}{l}<.824 E+01 \\
<.824 E+0 ?\end{array}$ & $\begin{array}{l}<.121 E-\{1 \\
<.121 E-11\end{array}$ & $\begin{array}{l}<.339 E+00 \\
<.339 E+00\end{array}$ & $\begin{array}{l}<.584 E-01 \\
<.584 F-01\end{array}$ & $\begin{array}{l}<.152 E+01 \\
<.249 E+02 \\
<.265 E+02\end{array}$ \\
\hline $\begin{array}{l}\text { MONTHLY } \\
\text { MAXIMUM } \\
\text { CONC }\end{array}$ & & $\begin{aligned} & (U C I / M L) \\
M A Y & \\
< & 113 E-07\end{aligned}$ & $\begin{array}{l}\text { (UCT/ML) } \\
\text { MAY } \\
.938 E-07\end{array}$ & (UCI/ML) & $(U C I / M L)$ & (UCI/ML) & (UCI/ML) & $\begin{array}{l}\text { (UCI/ML) } \\
\text { JUL } \\
<.354 E-08\end{array}$ \\
\hline $\begin{array}{l}\text { AVERAGE } \\
\text { CONC }\end{array}$ & & $<.164 F-08$ & $<.468 F-07$ & & & & & $<.341 E-08$ \\
\hline
\end{tabular}


MONTHLY SUMMARY OF LIQUID WASTF DISCHARGED TO GROUND FROM $1 / 1$ TO $9 / 301973$ AT

DISFOSAL SITE 216-S-19 PONE WASTE STPFAM DESCRIPTION- PEDOX LAOORATORY CDOLING WATER AND SINK WASTES FROM JANUARY 1952 TO DATE.

\begin{tabular}{|c|c|c|c|c|c|c|c|c|}
\hline 1973 & $\begin{array}{l}\text { VOLUME } \\
\text { (L) }\end{array}$ & $\begin{array}{l}F U \\
(G M)\end{array}$ & $\begin{array}{l}\text { EETA } \\
\text { (CI) }\end{array}$ & $\begin{array}{l}S R-90 \\
\text { (CI) }\end{array}$ & $\begin{array}{l}R U-13) 6 \\
(C T)\end{array}$ & $\begin{array}{l}\text { CS }-137 \\
\text { (CI) }\end{array}$ & $\begin{array}{l}\text { (O-60 } \\
\text { (CI) }\end{array}$ & $\begin{array}{l}U \\
\text { (KGM) }\end{array}$ \\
\hline $\begin{array}{l}\text { JAN } \\
\text { FEB } \\
\text { MAR } \\
\text { APR } \\
\text { MAY } \\
\text { JUN } \\
\text { JUL } \\
\text { AUE } \\
\text { SEP } \\
\text { OCT } \\
\text { NOV } \\
\text { DEC } \\
\text { ADJ }\end{array}$ & $\begin{array}{l}.299 E+07 \\
.275 F+17 \\
.323 E+17 \\
.299 E+77 \\
.311 E+07 \\
.284 F+07 \\
.293 E+37 \\
.293 E+.77 \\
.284 E+67\end{array}$ & $\begin{array}{r}<.525 E-13 \\
<.55 E E-03 \\
.236 E-82 \\
<.976 F-.3 \\
<.814 E-83 \\
<.786 E-83 \\
<.549 E-13 \\
<.137 E-02 \\
<.423 F-03\end{array}$ & $\begin{array}{r}.111 E-63 \\
<.172 F-03 \\
<.112 F-0.3 \\
<.118 E-03 \\
<.159 E-33 \\
<.263 E-33 \\
<.144 E-03 \\
<.588 E-03 \\
<.197 E-03\end{array}$ & & & & & $\begin{array}{l}<.318 E-01 \\
<.275 E-01 \\
<.345 E-01 \\
<.177 E-01 \\
<.322 E-01 \\
<.295 E-01 \\
<.311 E-01 \\
<.296 E-01 \\
<.344 E-01\end{array}$ \\
\hline $\begin{array}{l}73 \text { SUM } \\
\text { PRF SUM } \\
\text { TOT SUM }\end{array}$ & $\begin{array}{l}.266 E+78 \\
.693 E+19 \\
.720 E+89\end{array}$ & $\begin{array}{r}<.905 E-r 2 \\
.275 E+82 \\
.205 E+02\end{array}$ & $\begin{array}{r}<.195 E-02 \\
.325 E+23 \\
.325 E+03\end{array}$ & $\begin{array}{l}<.246 E+01 \\
<.245 E+01\end{array}$ & $\begin{array}{l}\text { <. } 682 E+11 \\
\text { c. } 682 F+11\end{array}$ & $\begin{array}{l}<.227 E+01 \\
<.227 E+01\end{array}$ & $\begin{array}{l}<.563 E+100 \\
<.563 E+00\end{array}$ & $\begin{array}{l}<.263 E+00 \\
<.107 E+03 \\
<.108 E+03\end{array}$ \\
\hline $\begin{array}{l}73 \text { DERAY } \\
\text { PREDECAY } \\
\text { TOTDECAY }\end{array}$ & $\begin{array}{l}.266 F+88 \\
.693 F+19 \\
.722 E+19\end{array}$ & $\begin{array}{r}<.95-2 \\
\cdot 205 F+02 \\
\cdot 205 E+2\end{array}$ & $\begin{array}{r}<.1415-22 \\
.106 F+02 \\
.106 E+82\end{array}$ & $\begin{array}{l}<.197 E+01 \\
<.197 E+01\end{array}$ & $\begin{array}{l}<.481 E-01 \\
e .431 E-01\end{array}$ & $\begin{array}{l}<.187 E+01 \\
<.187 E+B 1\end{array}$ & $\begin{array}{l}<.220 E+00 \\
=.220 E+100\end{array}$ & $\begin{array}{l}<.268 E+00 \\
<.107 E+03 \\
<.108 E+03\end{array}$ \\
\hline $\begin{array}{l}\text { MONTHLY } \\
\text { MAXIMUM } \\
\text { CONC }\end{array}$ & & $\begin{array}{l}(U C I / M L) \\
M A P \\
.391 F-77\end{array}$ & $\begin{aligned} & (U C I / M L) \\
& A U G \\
< & .235 E-C 6\end{aligned}$ & $(U C I / M L)$ & (UCI/ML) & $(U C T / M L)$ & (UCI/ML) & $\begin{array}{l}\text { (UCI/ML) } \\
\text { SEP } \\
\times .404 E-08\end{array}$ \\
\hline $\begin{array}{l}\text { AVERAGE } \\
\text { CONC }\end{array}$ & & $<.186 E-37$ & $<.734 E-87$ & & & & & c.336E-08 \\
\hline
\end{tabular}


TABLE 28

MONTHLY SUMMARY OF LIQUID WASTE DISCHARGED TO GROUND FPOM $1 / 1$ TO $9 / 301973$ AT DTSPOSAL STTF 216-T-H PONC WASTE STRFAM DESSPIPTION- 242-T EVAPORATOP CONDENSFR CCOLING WATER SINCE JANUARY 1.1967.

\begin{tabular}{|c|c|c|c|c|c|c|c|c|}
\hline 1973 & $\begin{array}{l}\text { VOLUME } \\
\text { (L) }\end{array}$ & $\begin{array}{l}P U \\
(G M)\end{array}$ & $\begin{array}{l}\text { EETA } \\
\text { (CI) }\end{array}$ & $\begin{array}{l}S R-90 \\
\text { (C.T) }\end{array}$ & $\begin{array}{l}R U-106 \\
(C . I)\end{array}$ & $\begin{array}{l}C S-137 \\
\text { (CI) }\end{array}$ & $\begin{array}{l}\text { CO-60 } \\
(\mathrm{CI})\end{array}$ & $\begin{array}{l}U \\
(K G M)\end{array}$ \\
\hline $\begin{array}{l}\text { JAN } \\
\text { FEB } \\
\text { MAR } \\
\text { APR } \\
\text { MAY } \\
\text { JUN } \\
\text { JUL } \\
\text { AUG } \\
\text { SEP } \\
\text { OCT } \\
\text { NOV } \\
\text { DEC } \\
\text { ADJ }\end{array}$ & $\begin{array}{l}.433 F+88 \\
.391 F+08 \\
.175 F+78 \\
.199 E+8 \\
.317 E+88 \\
.122 E+88 \\
.512 E+67 \\
.354 F+68 \\
.281 E+8\end{array}$ & $\begin{array}{r}<.13 E F-63 \\
<.149 E-03 \\
<.145 F-13 \\
.416 F-03 \\
.211 E-02 \\
<.362 F-13 \\
<.195 E-13 \\
.784 F-83 \\
<.223 E-r 3\end{array}$ & $\begin{array}{r}.321 E-.2 \\
<.336 E-02 \\
.204 E-62 \\
.215 E-82 \\
.436 E-02 \\
.176 E-62 \\
.429 E-72 \\
.427 F-r_{2} \\
<.123 E-62\end{array}$ & & & & & $\begin{array}{l}<.411 E+0 C \\
<.400 E+00 \\
<.134 E+00 \\
<.205 E+00 \\
<.321 E+00 \\
<.135 E+00 \\
<.544 E-01 \\
<.375 E+00 \\
<.300 E+00\end{array}$ \\
\hline $\begin{array}{l}73 \text { DERAY } \\
\text { PREDECAY } \\
\text { TOTDECAY }\end{array}$ & $\begin{array}{l}.252 E+9 \\
.415 F+11 \\
.418 F+11\end{array}$ & $\begin{array}{l}<.452 E-62 \\
<.367 E+1 \\
<.357 E+81\end{array}$ & $\begin{array}{r}. .174 F-11 \\
.306 E+12 \\
.376 E+12\end{array}$ & $\begin{array}{l}<.511 E+01 \\
<.511 E+01\end{array}$ & $\begin{array}{l}<.107 E+30 \\
<.107 F+00\end{array}$ & $\begin{array}{l}.922 E+01 \\
.922 E+01\end{array}$ & $\begin{array}{l}<.156 E+01 \\
<.156 E+01\end{array}$ & $\begin{array}{l}<.239 E+01 \\
<.689 E+03 \\
<.693 E+03\end{array}$ \\
\hline
\end{tabular}

\begin{tabular}{|c|c|c|c|c|c|c|c|}
\hline $\begin{array}{l}\text { MONTHLY } \\
\text { MAXIMUM } \\
\text { CONC }\end{array}$ & $\begin{array}{l}\text { (UCI/ML) } \\
\text { MAV } \\
.439 E-88\end{array}$ & $\begin{array}{l}\text { (UCI/ML) } \\
\text { JUL } \\
\text { B3 }\end{array}$ & (UCI/ML) & (URT/ML) & (UCI/ML) & $(U C I / M L)$ & $\begin{array}{l}\text { (UCI/ML) } \\
\text { JUN } \\
\text { e. } 367 E-08\end{array}$ \\
\hline AVERAGE & & 1 & & & & & \\
\hline CONG & $<.11 \cap \mathrm{E}-\mathrm{CB}$ & $<.106 F-16$ & & & & & $<.315 E-08$ \\
\hline
\end{tabular}

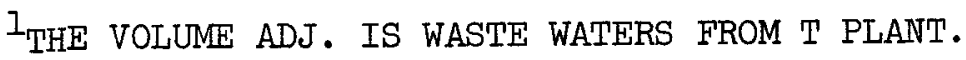


MONTHLY SUMMARY DF LIQUID WASTE CISCHARGEO TO GROUND FPOM $1 / 1$ TO $9 / 301973$ AT DISPNSAL SIT = 21E-UI-1N SUMMARY

WASTE STRFAM TESCPIFTION- U PLANT ANS 241-SX COOLING WATER, 234-5 AND 231-Z WASTE (Z-11 AND Z-19 DITCHES), LAUADPY WASTE ANO POWER HOUSE WATER.

\begin{tabular}{|c|c|c|c|c|c|c|c|c|}
\hline 1973 & $\begin{array}{l}\text { VOLUMF } \\
\text { (L) }\end{array}$ & $\begin{array}{l}r u \\
(G y)\end{array}$ & $\begin{array}{l}\text { PFTA } \\
\text { (CI) }\end{array}$ & $\begin{array}{l}S P-90 \\
(C I)\end{array}$ & $\begin{array}{l}R U-1: 5 \\
\text { (CI) }\end{array}$ & $\begin{array}{l}\operatorname{cs}-137 \\
(C I)\end{array}$ & $\begin{array}{l}C 0-60 \\
(C I)\end{array}$ & $\begin{array}{l}U \\
\text { (KGM) }\end{array}$ \\
\hline $\begin{array}{l}\text { JAN } \\
\text { FEB } \\
\text { MAR } \\
\text { APR } \\
\text { MAY } \\
\text { JUN } \\
\text { JUL } \\
\text { AUG } \\
\text { SEP } \\
\text { OCT } \\
\text { NOV } \\
\text { DEC } \\
\text { ADJ }\end{array}$ & $\begin{array}{l}.739 F+08 \\
.587 E+: 8 \\
.740 F+8 \\
.716 E+88 \\
.688 F+8 \\
.792 E+88 \\
.766+8 \\
.749 E+38 \\
.8175+8\end{array}$ & 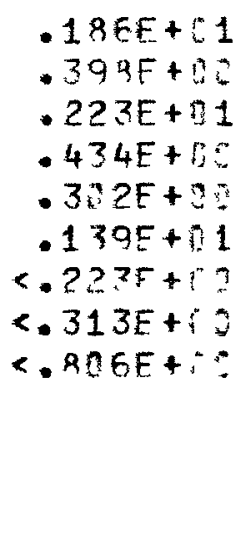 & $\begin{array}{r}.346 E-81 \\
\times .156 E-.1 \\
.895 F-11 \\
<.203 E-\$ 1 \\
<.503 E-31 \\
<.126 E+30 \\
<.107 E+8 \\
<.866 E-11 \\
<.107 F+0\end{array}$ & & & & & $\begin{array}{l}r .257 E+00 \\
<.232 E+00 \\
<.256 E+0 \\
r .199 E+00 \\
<.152 E+00 \\
<.149 E+00 \\
<.160 E+00 \\
<.162 E+00 \\
r .149 E+00\end{array}$ \\
\hline $\begin{array}{l}73 \text { SUM } \\
\text { PRE SUM } \\
\text { TOT SUM }\end{array}$ & $\begin{array}{l}\cdot 122 E+10 \\
\cdot 119 F+12 \\
\cdot 121 F+12\end{array}$ & $\begin{array}{r}<.795 E+.1 \\
.81 C E+.4 \\
.811 E+.4\end{array}$ & $\begin{array}{r}<.635 E+30 \\
.305 E+84 \\
.305 E+84\end{array}$ & $\begin{array}{l}<.226 E+02 \\
\text { <. } 226 E+02\end{array}$ & $\begin{array}{l}.309 F+13 \\
.309 F+13\end{array}$ & $\begin{array}{l}<.157 E+02 \\
<.157 E+02\end{array}$ & $\begin{array}{l}<.435 E+01 \\
<.435 E+.1\end{array}$ & $\begin{array}{r}<.172 E+01 \\
.144 E+04 \\
.144 E+04\end{array}$ \\
\hline $\begin{array}{l}73 \text { DECAY } \\
\text { PREDECAY } \\
\text { TOTDECAY }\end{array}$ & $\begin{array}{l}122 E+10 \\
\cdot 119 E+12 \\
\cdot 121 F+12\end{array}$ & $\begin{array}{r}.795 E+C 1 \\
.81 C E+C 4 \\
.911 F+14\end{array}$ & $\begin{array}{r}<.453 E+50 \\
.670 E+82 \\
.675 E+2\end{array}$ & $\begin{array}{l}<.161 E+\pi 2 \\
<.161 E+02\end{array}$ & $\begin{array}{l}<.193 E+00 \\
<.193 E+00\end{array}$ & $\begin{array}{l}<.115 E+C 2 \\
<.115 E+02\end{array}$ & $\begin{array}{l}<.193 F+C 1 \\
<.193 E+11\end{array}$ & $\begin{array}{r}<.172 E+01 \\
.144 E+04 \\
.144 E+04\end{array}$ \\
\hline $\begin{array}{l}\text { MONTHLY } \\
\text { MAXIMUM } \\
\text { CONC }\end{array}$ & & $\begin{array}{l}\text { (URI/ML) } \\
\text { MAR } \\
.18 E E-05\end{array}$ & $\begin{array}{l}\text { (UCI/ML) } \\
\text { JUN } \\
<=15 Q E-05\end{array}$ & $(U C I / M L)$ & $(U C I / M L)$ & $(U C I / M L)$ & $(U O I / M L)$ & $\begin{array}{l}\text { (UCI/ML) } \\
\text { JAN } \\
\times \cdot 116 E-08\end{array}$ \\
\hline $\begin{array}{l}\text { AVERAGE } \\
\text { CONC }\end{array}$ & & $<.402 E-C 6$ & $<.523 E-06$ & & & & & $<.470 E-09$ \\
\hline
\end{tabular}

${ }^{1}$ VOLUME ADJ. INCLUDES . $509+09$ FROM 200 WEST POWERHOUSE AND . 372 + 08 FROM 221-U. 
MONTHLY SUMMARY OF LIQUID WASTE DISCHARGED TO GROUND FROM $1 / 1$ TO $9 / 301973$ AT DISPOSAL SITF 21F-U-10 DITCH WASTE STREAM DFSCRIPTINN- LAUNCRY WASTE WATER (U-14 JITCH), SINCE JANUARY $1,1963$.

\begin{tabular}{|c|c|c|c|c|c|c|c|c|}
\hline 1973 & $\begin{array}{l}\text { VOLUME } \\
\text { (L) }\end{array}$ & $\begin{array}{l}P U \\
(G M)\end{array}$ & $\begin{array}{l}\text { PETA } \\
\text { (CI) }\end{array}$ & $\begin{array}{l}S 2-93 \\
\text { (SI) }\end{array}$ & $\begin{array}{l}\text { RU-136 } \\
\text { (CI) }\end{array}$ & $\begin{array}{l}\mathrm{CS}-137 \\
(\mathrm{CI})\end{array}$ & $\begin{array}{l}\mathrm{CO}-60 \\
\text { (CI) }\end{array}$ & $U_{(K G M)}$ \\
\hline $\begin{array}{l}\text { JAN } \\
\text { FEB } \\
\text { MAR } \\
\text { APR } \\
\text { MAY } \\
\text { JUN } \\
\text { JUL } \\
\text { AUG } \\
\text { SEP } \\
\text { OCT } \\
\text { NOV } \\
\text { DEC } \\
\text { ADJ }\end{array}$ & $\begin{array}{l}.240 F+07 \\
.217 E+07 \\
.17 F E+.7 \\
.402 E+.77 \\
.557 E+07 \\
.744 E+.7 \\
.604 E+37 \\
.643 E+.57 \\
.525 F+07\end{array}$ & $\begin{array}{r}<.248 E-02 \\
<.291 E-03 \\
<.45 . E-63 \\
.975 E-13 \\
.134 E-62 \\
<.107 E-62 \\
<.116 E-03 \\
.122 E-.3 \\
<.770 E-03\end{array}$ & $\begin{array}{l}.525 E-03 \\
.142 E-02 \\
.577 E-73 \\
.223 E-02 \\
.321 E-02 \\
.170 E-11 \\
.187 E-31 \\
.538 E-\$ 2 \\
.951 E-03\end{array}$ & & & & & \\
\hline $\begin{array}{l}73 \text { SUM } \\
\text { PRE SUM } \\
\text { TOT SUM }\end{array}$ & $\begin{array}{l}.411 F+08 \\
.243 F+89 \\
.284 E+.99\end{array}$ & $\begin{array}{l}<.75 L F-C 2 \\
<.353 E+C \\
<.351 E+00\end{array}$ & $\begin{array}{r}.499 E-[1 \\
<.378 E+11 \\
<.393 E+1\end{array}$ & & & & & \\
\hline $\begin{array}{l}73 \text { DFCAY } \\
\text { PREDECAY } \\
\text { TOTDECAY }\end{array}$ & $\begin{array}{l}.411 \pi+79 \\
.243 E+.9 \\
.284 E+: 9\end{array}$ & $\begin{array}{l}<.754 E-.2 \\
<.353 E+.3 \\
<.361 E+.\end{array}$ & $\begin{array}{r}.357 F-11 \\
<.243 E+00 \\
<.278 E+-C\end{array}$ & & & & & \\
\hline $\begin{array}{l}\text { MONTHLY } \\
\text { MAXIMUM } \\
\text { CONC }\end{array}$ & & $\begin{array}{l}(U C I / M L) \\
\text { JAN } \\
<.636 E-D 7\end{array}$ & $\begin{array}{l}(U C I / M L) \\
\text { JUL } \\
.309 E-05\end{array}$ & $(U C I / M L)$ & (UCI/ML) & $(U C I / M L)$ & $(U C I / M L)$ & $(U C I / M L)$ \\
\hline $\begin{array}{l}\text { AVERAGE } \\
\text { CONC }\end{array}$ & & $<.113 E-[17$ & $.122 E-35$ & & & & & \\
\hline
\end{tabular}


TABLE 31

MONTHLY SUMMARY OF LIRUID WASTE DISCHARGED TO GROUNO FROM $1 / 1$ TO $9 / 301973$ AT DISPOSAL STTF 21E-U-10 DITCH WASTE STREAM DESCRIFTIOA- 241-SX CCOLING WATFR, SINCE JANUARY 1.1968.

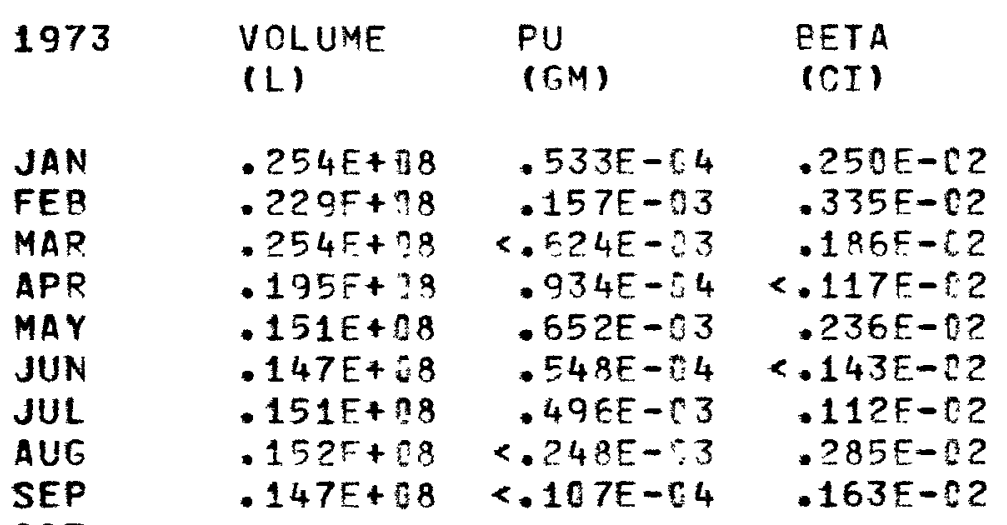

\begin{tabular}{|c|c|c|c|c|}
\hline $\begin{array}{l}S R-90 \\
(C I)\end{array}$ & $\begin{array}{l}R U-106 \\
(C I)\end{array}$ & $\begin{array}{l}\text { CS-137 } \\
(\mathrm{CI})\end{array}$ & $\begin{array}{l}(0-60 \\
(\mathrm{CI})\end{array}$ & $\begin{array}{l}U \\
(K G M) \\
\\
<.257 E+00 \\
<.232 E+00 \\
<.256 E+00 \\
<.199 E+00 \\
<.152 E+00 \\
<.149 E+00 \\
<.160 E+00 \\
<.162 E+00 \\
<.149 E+00\end{array}$ \\
\hline
\end{tabular}

NOV

DEC

ADJ

$\begin{array}{ll}73 \text { SUM } & .158 E+r 9 \\ \text { PRE SUM } & .831 E+09 \\ \text { TOT SUM } & .999 F+09 \\ & \\ 73 \text { RECAY } & .1595+99 \\ \text { PREDECAY } & .831 E+09\end{array}$

$<.239 E-12<.183 E-81$

$<.644 E+2<.207 E+? 2$

$<.644 F+02<.207 E+02$

$<.498 E+00$

$<.111 \mathrm{E}+11$

$<.117 E+01$

$<.361 E+0 ?$

$<.172 E+01$

$<.389 E+01$

$<.239 F-32<.117 E-r 1$

C. E44F+

$<.37 L E+D 1$

$<.498 E+00$

$<.1115+01$

$<.117 E+61$

$<.351 E+03$

$<.561 E+01$

TOTOECAY . O99E+19

$<.644 E+32$

$<.371 E+01$

$<.441 E+00$

<. $3[8 E-C 1$

$<.105 E+01$

$<.181 E+00$

C.172E+01

$<.561 E+B 1$

\section{MONTHLY \\ MAXIMUM \\ CONC}

$$
\begin{array}{ll}
\text { (UCI/ML) } & \text { (UCI/ML) } \\
\text { MAY } & \text { AUG } \\
.264 F-188 & .187 E-66 \\
& \\
<.373 E-69 & <.109 E-? 6
\end{array}
$$

(UCI/ML)

(UCI/ML)

IUCI/ML)

(UCI /ML)

(UCI/ML)

AUG

$<.354 E-08$

AVERAGE

CONC

$<.340 E-08$ 
MONTHLY SUMMARY OF LIQUID HASTE DISCHARGEO TO GROUND FROM $1 / 1$ TO $9 / 301973$ AT OISPRCBL SITF 215-U-10 DITCH WASTE STR=AH DESOPIFTION- $231-Z$ COCLING WATFP (7-19 DITCH FROM JANUARY 1972 TO OATE)

\begin{tabular}{|c|c|c|c|}
\hline 1973 & $\begin{array}{l}\text { VOLUME } \\
\text { (L) }\end{array}$ & $\begin{array}{l}P U \\
(6 M)\end{array}$ & $\begin{array}{l}\text { PETA } \\
\text { (CI) }\end{array}$ \\
\hline
\end{tabular}

$\begin{array}{llrl}\text { JAN } & .213 E+[8 & <.299 E-B 2 & <.256 E-02 \\ \text { FEB } & .212 E+C 8 & <.447 E-02 & <.361 E-02 \\ \text { MAR } & .213 F+89 & .730 E-[2 & <.364 F-0 ? \\ \text { APR } & .213 F+8 & <.438 E-02 & <.32 C E-02 \\ \text { MAY } & .214 E+08 & .443 E-02 & <.289 E-02 \\ \text { JUN } & .223 E+08 & <.313 E-02 & <.296 E-02 \\ \text { JUL } & .224 E+08 & <.311 E-03 & <.153 E-02 \\ \text { AUG } & .2245+08 & .345 E-03 & <.994 E-73 \\ \text { SEP } & .222 E+08 & <.335 E-02 & <.138 E-C 2\end{array}$

OCT

NOV

DEC

$A D J$

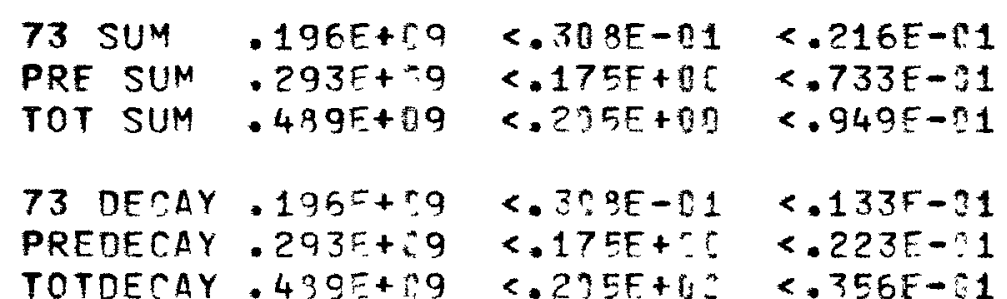

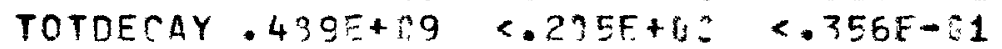

MONTHLY

MAXIMUM

CONC

\section{AVERAGE}

CONC

(UCI/ML) $\quad$ (UCI/ML) (UCI/ML) (UCI/ML) (UCI/ML) (UCI/ML) (UCI/ML)
MAP
$.212 F-07<.150 E-E 6$


MONTHLY SUMMARY OF LIQUID HASTE OISCHARGFD TO GROUND FRCM $1 / 1$ TO $9 / 301973$ AT DISFOSAL STTF 216-1)-10 DITCH

WASTE STRFAM TESCRIPTIOA- $234-5$ COCLING WATER FROM JANLARY 1, 1958 TO CATE ANC THE $231-2$ CONLING WATER FROM JANUARY 1,1968 TO DECEMEER 31,1971 . (Z-11 DITCH FROM JANUAPY 1, 1968 TO MAY 1971 AND Z-19 DITCH FROM MAY 1971 TO DATE)

\begin{tabular}{|c|c|c|}
\hline 1973 & $\begin{array}{l}\text { VOLUME } \\
\text { (L) }\end{array}$ & $\begin{array}{l}P U \\
(G M)\end{array}$ \\
\hline
\end{tabular}

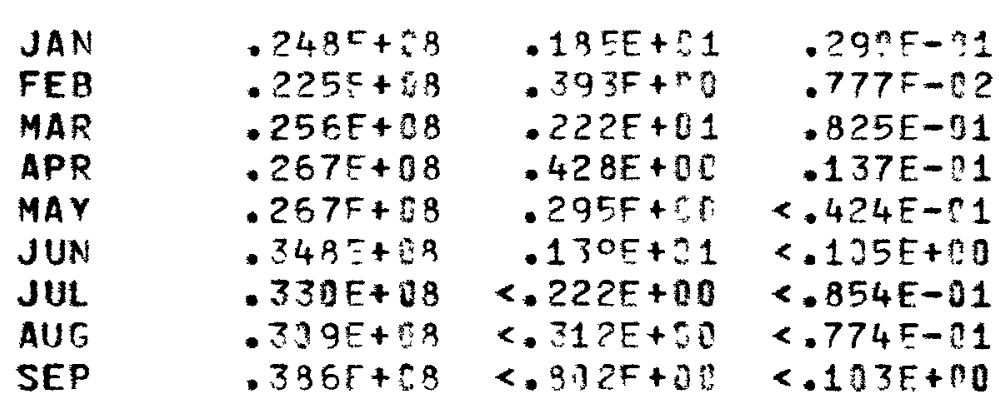

OCT

NOV

DEC

ADJ

\section{SUM $.264 F+09$ \\ PRE SUM \\ TOT SUM \\ $245 F+10$ \\ - $272 E+1$ ? \\ 73 DECAY . 264F+09 \\ PREDERAY -245E+10}

TOTDECAY - 2??E+10
$<.791 F+01$

$<.87 P E+\cap 2$

$<.951 F+12$

$<.7915+01$

$<.872 E+02$

$<.951 F+.2$

$<.792 F+01$

$<.931 E+1$

$<.546 E+00$

$<.556 E+D 2$

$<.561 \mathrm{Ft} 2$

<. $222 E+01$

$<.222 E+11$

<. 200E+01

$<.2 R 3 E+01$
MONTHLY

MAXIMUM

CONC

AVERAGF

CONC
MAR

$.532 \mathrm{E}-05 \quad \cdot 322 \mathrm{~F}-? 5$

$<.184 E-15<.267 F-35$
(UCI/ML)

(UCI/ML)

(UCI/ML)

(UCI /ML)

(UCI/ML) 
1. HW-5517E - WOND, V. W., (INDEX OF CPO CRIB BUILDING NUMBERS, DESIGNS OF CPC RACIOACTIVE LIQUID WASTF TISPOSAL SITES), MARCH 1E, 1958 , UNCLASSIFIER.

2. ARH-1562 - LUNDGPEA, L. L., 120 F FAST AND ACPTH AREAS RADICACTIVE LIQUTD WASTE DISFOSAL SITESI, JANUARY 1, 1970, UNCLASSIFIED.

3. ARH-2155 - LUNDGPEN, L. L., IPANIOACTIVE LIQUIS, WASTE DISFCSAL FACILITIES IN THE 230 KEST AREA), AUGUST 31, 1971, UNCLASSIFIEC.

4. APR-2751 - ANDEPSCN, J. D., HANSON, G. L., KIEL, G. Z., MCMURRY, 3. J., DNE VTSICK, N. P., IINPUT AND DECAYED VALUES OF RADIOACTIVE LIQUID WASTES DISCHARGED TO THE GROUND IN THE 2 CO AREAS THROUGH 1971), MARCH ?2, 1973, UNCLASSIFIED.

5. ARH-2757 - DART 3. ANDFRSON, J. ח., IRADIOACTIVE LIQUIC WASTES JISCHARGED TO GRCUN IN THE 2DC AREAS DURING 19721, APRIL 9, 1973, UNCLASSIFIED. 